Florida International University FIU Digital Commons

9-21-2017

\title{
Quantitative Yttrium-90 Bremsstrahlung SPECT/ CT and PET/CT Study for 3D Dosimetry in Radiomicrosphere Therapy
}

Senait Aknaw Debebe

Department of Biomedical Engineering, Florida International University, sdebe002@fiu.edu

DOI: $10.25148 /$ etd.FIDC004028

Follow this and additional works at: https://digitalcommons.fiu.edu/etd

Part of the Analytical, Diagnostic and Therapeutic Techniques and Equipment Commons, Bioimaging and Biomedical Optics Commons, and the Biomedical Commons

\section{Recommended Citation}

Debebe, Senait Aknaw, "Quantitative Yttrium-90 Bremsstrahlung SPECT/CT and PET/CT Study for 3D Dosimetry in Radiomicrosphere Therapy" (2017). FIU Electronic Theses and Dissertations. 3538.

https://digitalcommons.fiu.edu/etd/3538 


\section{FLORIDA INTERNATIONAL UNIVERSITY}

Miami, Florida

\section{QUANTITATIVE YTTRIUM-90 BREMSSTRAHLUNG SPECT/CT AND PET/CT STUDY FOR 3D DOSIMETRY IN RADIOMICROSPHERE THERAPY}

A dissertation submitted in partial fulfillment of the

requirements for the degree of

DOCTOR OF PHILOSOPHY

in

BIOMEDICAL ENGINEERING

by

Senait Aknaw Debebe

2017 


\section{To: Dean John Volakis}

College of Engineering and Computing

This dissertation, written by Senait Aknaw Debebe, and entitled Quantitative Yttrium-90 Bremsstrahlung SPECT/CT and PET/CT Study for 3D Dosimetry in Radiomicrosphere Therapy, having been approved in respect to style and intellectual content, is referred to you for judgment.

We have read this dissertation and recommend that it be approved.

Juan Franquiz

Malek Adjouadi

Wei-Chiang Lin

Shuliang Jiao

Anthony J. McGoron, Major Professor

Date of Defense: September 21, 2017

The dissertation of Senait Aknaw Debebe is approved.

Dean Jonh Volakis

College of Engineering and Computing

Andrés G. Gil

Vice President for Research and Economic Development And Dean of the University Graduate School

Florida International University, 2017 


\section{DEDICATION}

To my sons, Yared and Matthias, you are my shining stars, my inspiration and my strength, you make me better and more fulfilled than I could ever imagined. I love you dearly. 


\section{ACKNOWLEDGMENT}

I would like to express my sincere gratitude to my advisor Dr. Anthony McGoron. I thank him for his tremendous mentorship and providing me with every opportunity to grow academically and emotionally. I am grateful for his patience and giving me the stability and freedom I needed over the years. To Dr. Juan Franquiz, who has taken much time to work with me on every experiment and led me to completion of my dissertation, thank you for your time and professional mentorship.

I want to thank my dissertation committee members: Dr. Malek Adjouadi for his guidance throughout my dissertation work, directing me to the right tools and methodologies; Dr. Wei-Ching Lin for his financial support to help me buy the chemicals required for my dissertation work and his valuable advice; Dr. Shuliang Jiao for his insightful suggestions to improve my work. To Dr. Seza Gulec, although not officially my committee member, his contribution to my paper with his expert knowledge in radiotherapy and nuclear medicine is paramount.

I would also like to acknowledge the Baptist Hospital of Miami for allowing me to use the imaging devices to perform experiments. My special thanks to the Imaging department for their willingness to help during my experiments and answer all my bewildering questions.

Finally, I want to thank my family for their love, support, sacrifice and unwavering belief in me. My mom, your willingness to listen to all my frustrations and your persistent advice has gotten me through the hard times. My brother, Teddy, thank you for keeping my spirits high during the tough times, I wouldn't have made it without you reminding me I can do it. To my husband, Shimelis Dessu, I am truly grateful for all your help. To my dearest kids, Yared and Matthias, for your endurance for the many long hours of my absence to complete this dissertation. Above all, I thank God for helping me finish the dissertation journey. 


\title{
ABSTRACT OF THE DISSERTATION \\ QUANTITATIVE YTTRIUM-90 BREMSSTRAHLUNG SPECT/CT AND PET/CT STUDY FOR 3D DOSIMETRY IN RADIOMICROSPHERE THERAPY
}

\author{
by \\ Senait Aknaw Debebe \\ Florida International University, 2017 \\ Miami, Florida \\ Professor Anthony J. McGoron, Major Professor
}

Liver cancer ranks the third most common cause of cancer related mortality worldwide. Radiomicrosphere therapy (RMT), a form of radiation therapy, involves administration of Yttrium$90\left({ }^{90} \mathrm{Y}\right)$ microspheres to the liver via the hepatic artery. ${ }^{90} \mathrm{Y}$ microspheres bremsstrahlung SPECT/CT or PET/CT imaging could potentially identify an extrahepatic uptake. An early detection of such an uptake, thus, could initiate preventative measures early on. However, the quantitative accuracy of bremsstrahlung SPECT/CT images is limited by the wide and continuous energy spectrum of ${ }^{90} \mathrm{Y}$ bremsstrahlung photons. ${ }^{90} \mathrm{Y}$ PET/CT imaging is also possible but limited by the extremely small internal pair production decay. These limitation lead to inaccurate quantitation of microsphere biodistribution especially in small tumors.

SPECT/CT and PET/CT acquisition of a Jasczak phantom with eight spherical inserts filled with ${ }^{90} \mathrm{Y}_{3} \mathrm{Cl}$ solution were performed to measure the quantitative accuracy of the two imaging modalities. ${ }^{90} \mathrm{Y}$ microsphere SPECT/CT data of 17 patients who underwent RMT for primary or metastatic liver cancer were acquired. Technetium-99m macroaggregated albumin $\left({ }^{99 \mathrm{~m}} \mathrm{Tc}-\mathrm{MAA}\right)$ SPECT/CT scans were also collected, but available for only twelve of the patients. SPECT/CT images from phantoms were used to determine the optimal iteration number for the iterative spatial resolution recovery algorithm. Methods for image based calculation of calibration factors for 
activity estimation from the patient and phantom ${ }^{90} \mathrm{Y}$ bremsstrahlung SPECT/CT images were developed. Tumor areas were segmented using an active contour method. The ${ }^{99 \mathrm{~m} T c-M A A}$ and ${ }^{90} \mathrm{Y}$ microsphere SPECT/CT images were co-registered a priori for correlation analysis. Comparison of uptake on ${ }^{99 \mathrm{~m} T c-M A A}$ and ${ }^{90} \mathrm{Y}$ microsphere SPECT/CT images was assessed using tumor to healthy liver ratios. Furthermore, a three dimensional absorbed dose estimation algorithm was developed using the voxel S-value method. Absorbed doses within the tumor and healthy part of the liver were investigated for correlation with administered activity.

Improvement in contrast to noise ratio and contrast recovery coefficients $\left(\mathrm{Q}_{\mathrm{H}}\right)$ on patient and phantom ${ }^{90} \mathrm{Y}$ bremsstrahlung SPECT/CT images as well as PET/CT images were achieved. Total activity estimations in liver and phantom gave mean percent errors of $-4 \pm 12 \%$ and $-23 \pm$ $41 \%$ for patient and phantom SPECT/CT studies. The pre and post-treatment images showed significant correlation $(r=0.9, p<0.05)$ with mean TLR of $9.2 \pm 9.4$ and $5.0 \pm 2.2$ on ${ }^{99 m}$ Tc-MAA and ${ }^{90} \mathrm{Y}$ microspheres SPECT/CT respectively. The correlation between the administered activity and tumor absorbed dose was weak $(\mathrm{r}=0.5, \mathrm{p}>0.05)$, however, healthy liver absorbed dose increased with administered activity $(\mathrm{r}=0.8, \mathrm{p}<0.05)$. 


\section{TABLE OF CONTENTS}

\section{CHAPTER}

PAGE

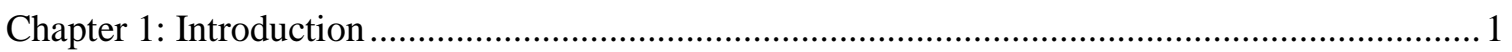

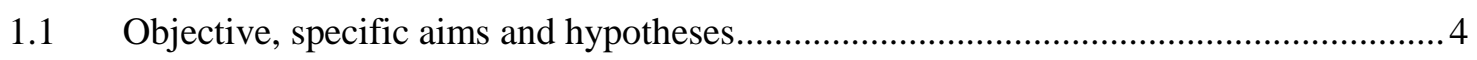

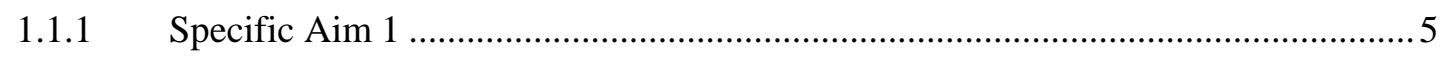

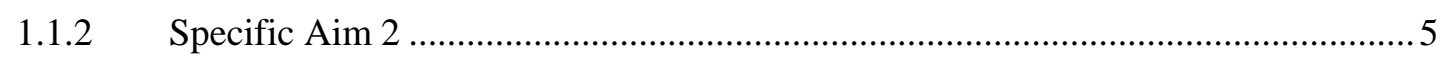

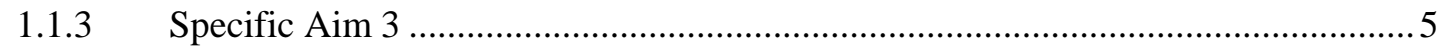

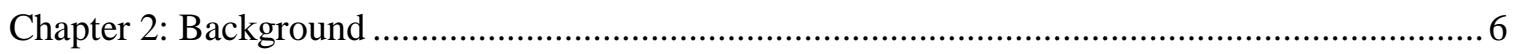

2.2 Nuclear Medicine Imaging Modalities in Liver Cancer Management.................................. 6

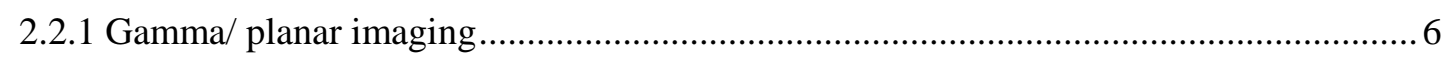

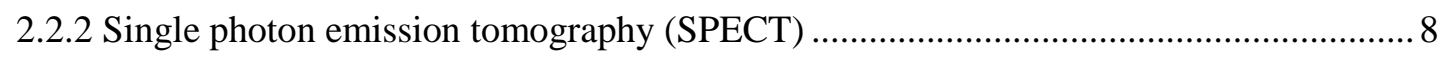

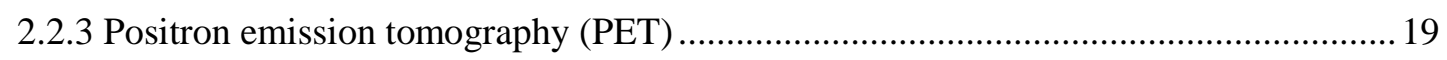

Chapter 3: Framework for Experimental Setup, Data Acquisition and Data Collection ...............23

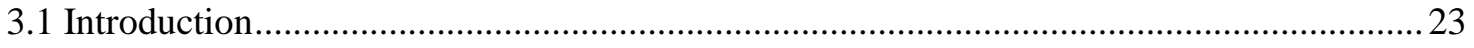

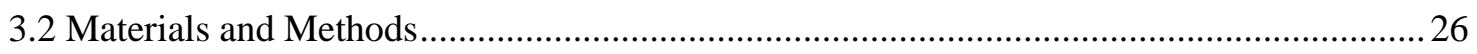

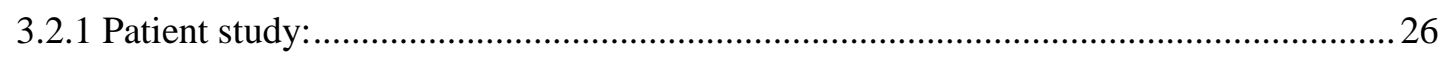

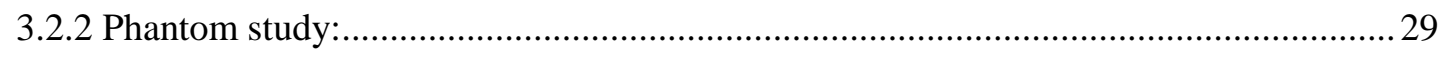

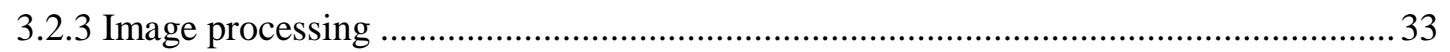

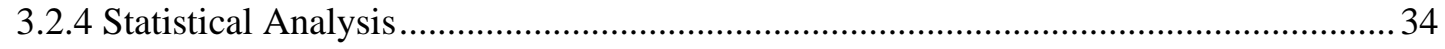

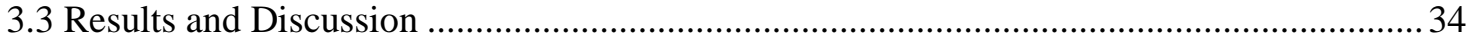

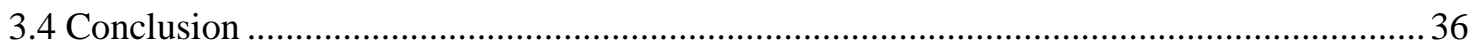

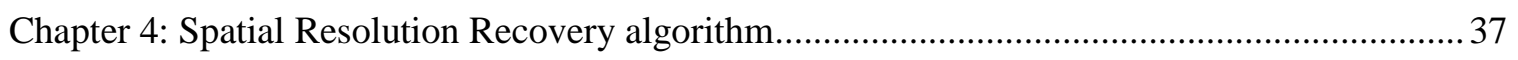

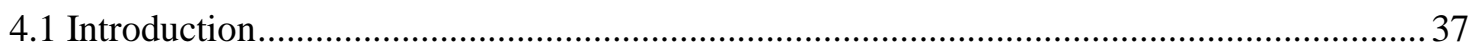

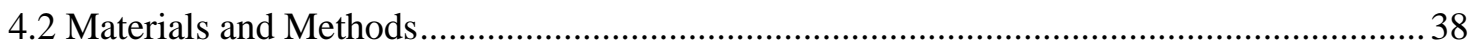

4.2.1 The Richardson - Lucy deconvolution method ............................................................ 38

4.2.2 Modeling of the point spread function of the detector ................................................. 39

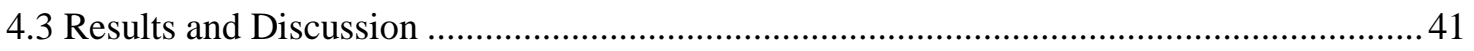

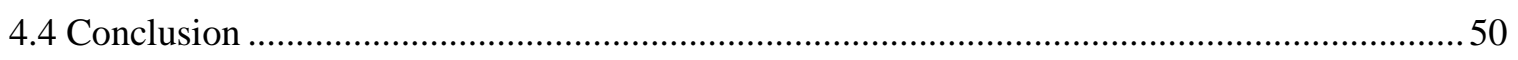

Chapter 5: Calibration factor and Activity estimation ............................................................. 50

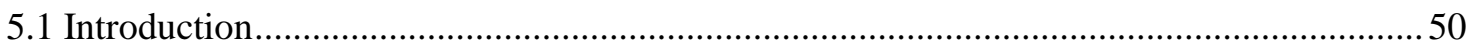

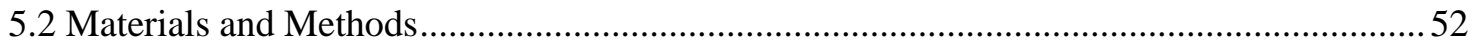




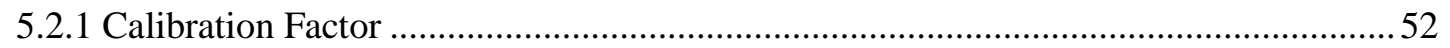

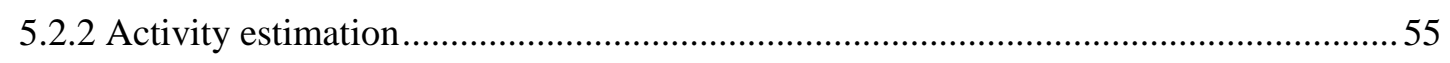

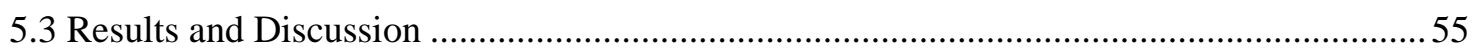

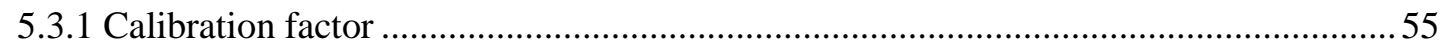

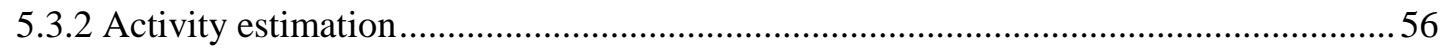

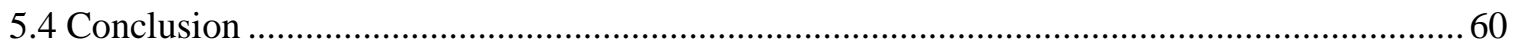

Chapter 6: Comparison of tumor to healthy liver ratio on ${ }^{99 \mathrm{~m}} \mathrm{Tc}-\mathrm{MAA}$ vs. ${ }^{90} \mathrm{Y}$ microsphere

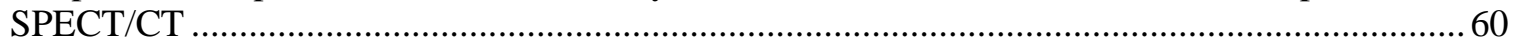

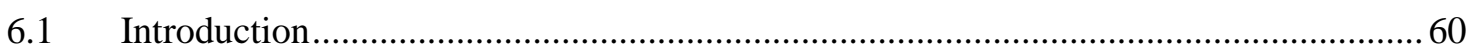

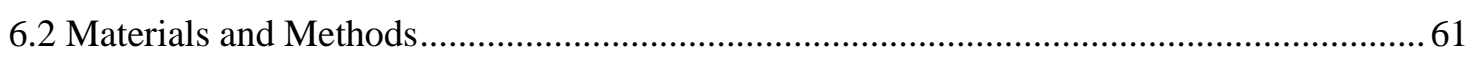

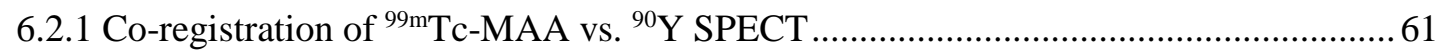

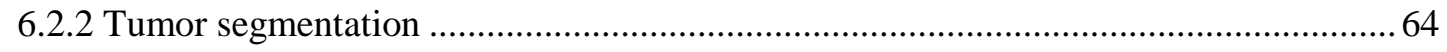

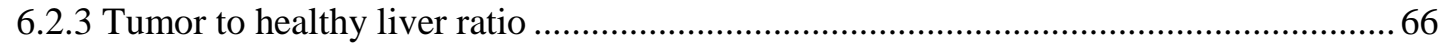

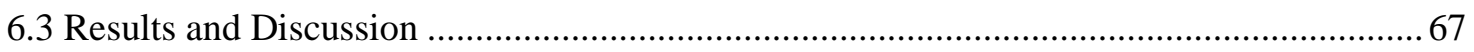

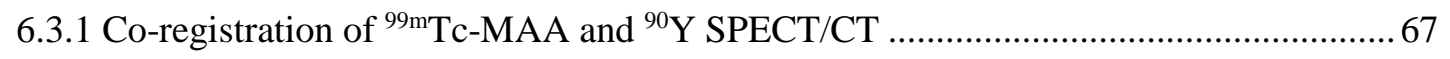

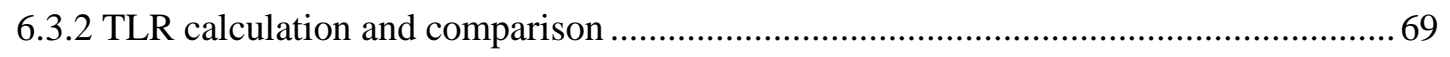

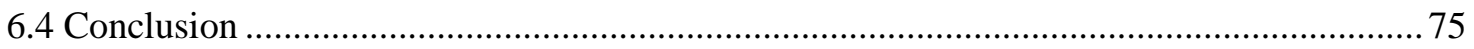

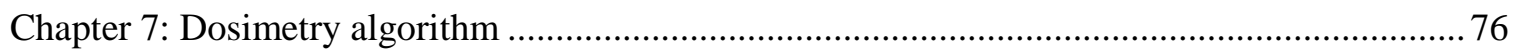

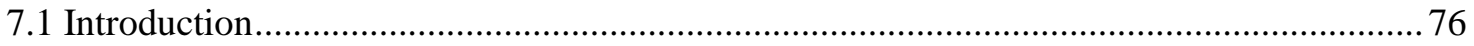

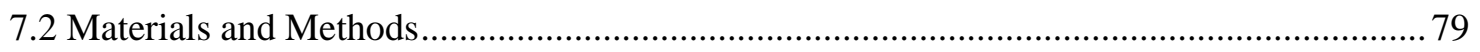

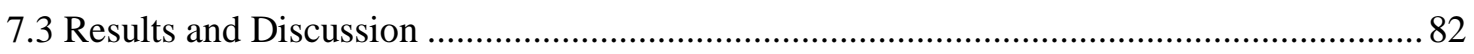

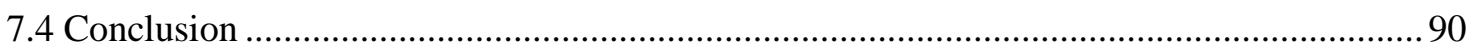

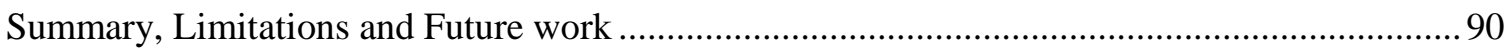

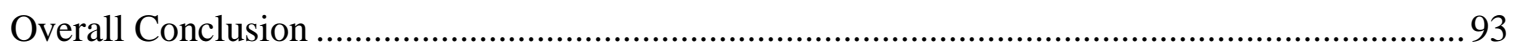

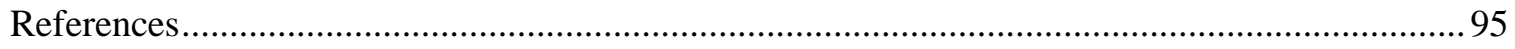

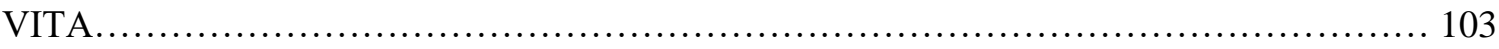




\section{LIST OF TABLES}

TABLE

PAGE

Table 1: Collimator specification at $10 \mathrm{~cm}$ from collimator face (4) . ......................................... 7

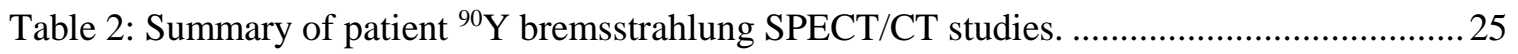

Table 3: Summary of ${ }^{90} \mathrm{Y}$ bremsstrahlung SPECT/CT phantom study setups..............................25

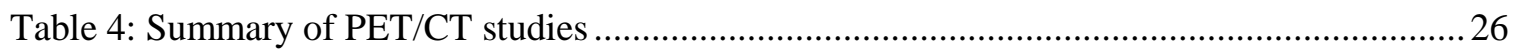

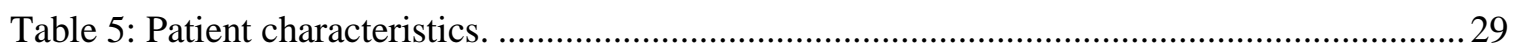

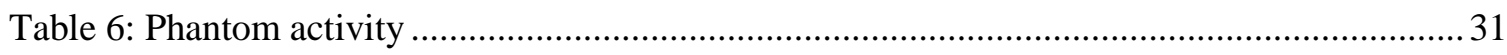

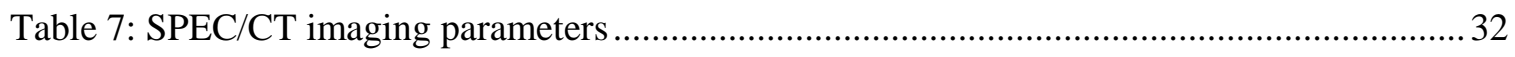

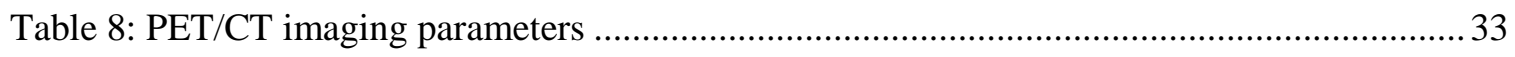

Table 9: QH calculated using the ROI and VOI method for the sphere sizes .............................. 43

Table 10: Administered activities and total estimated activities inside liver VOI.......................57

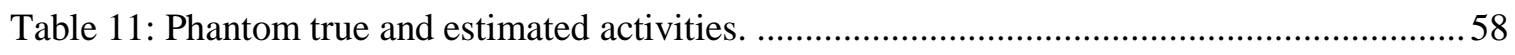

Table 12: ANOVA for the choice of collimator and FWHM ..................................................59

Table 13: Minitab result of paired T-test between pre and post-treatment tumor volumes........... 71

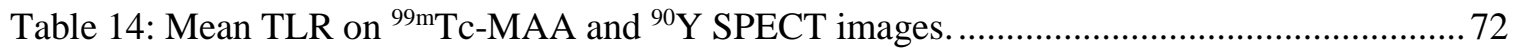

Table 15: Tumor volumes and TLRs for pre and post-treatment SPECT/CT images. ..................86 


\section{LIST OF FIGURES}

\section{FIGURE}

Figure 1:(I) RMT procedure: (A) introduction of a catheter into the femoral artery, (B) injection of microspheres, (C) liver vasculature (9). (II) Process diagram of RMT.

Figure 2: Various positions of a catheter tip during pretreatment stage and associated with unintended distribution (12)....

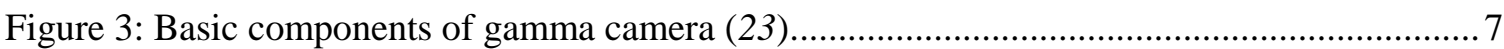

Figure 4: Example of planar images with ROIs drawn over the anterior (a) and posterior (b) of

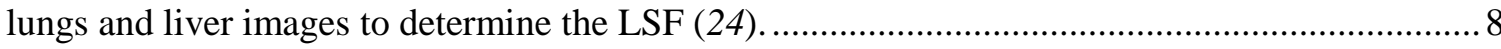

Figure 5: SPECT/CT camera (left) and its schematic (right)................................................... 10

Figure 6: SPECT image formation (left) and 2D image reconstruction (right) (26)..................... 10

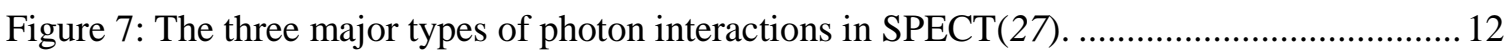

Figure 8: Profile of a parallel hole collimator for a point source (23) ….................................... 13

Figure 9: (A) Reference anatomic model used for conventional dose calculation (32) and (B)

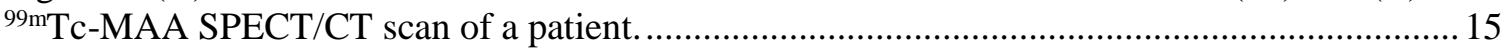

Figure 10: Schematic representation of the energy levels (43) and energy spectrum of ${ }^{90} \mathrm{Y}(8) \ldots 18$

Figure 11: Equal probability assigned to all the pixels along LOR in non-TOF PET (a) and

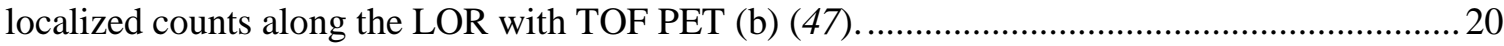

Figure 12: The process of positron-electron annihilation and detection of the annihilation photons (left) and illustration of PET/CT scanner components (48) (right)................................21

Figure 13: Positron fraction over the continuous bremsstrahlung spectrum as measured by

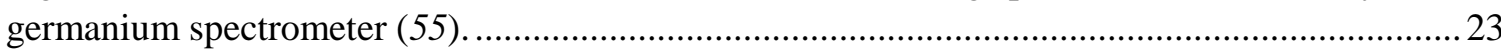

Figure 14: Patient ${ }^{90} \mathrm{Y}$ microsphere SPECT/CT images in axial (upper) and coronal (lower) views.

Figure 15: Different size hollow spheres (left) and Jasczak phantom with spherical inserts (right)

Figure 16: SPECT/CT (left) Phantom imaging, and energy resolution of the two detectors centered for the MEGP (middle) and HEGP (right).

Figure 17: CT (left) and SPECT reconstructed with Astonish OSEM MEGP, OSEM only MEGP and Astonish OSEM HEGP consecutively of experiment 1. 
Figure 18: CT (left) and SPECT (right) scans imaged with the MEGP collimator of experiment 2.

Figure 19: PECT/CT scanner and CT image (upper) and PET 15 \& 30min images (lower). ........36

Figure 20: Block diagram of the RL algorithm employed.

Figure 21: Phantom image qualities for the MEGP and HEGP collimators. Column numbers correspond to the iterations number of the algorithm and rows refer to the FWHM of the PSF... 42

Figure 22: ROI and VOI based contrast resolution coefficient for different iteration number, $\mathrm{FWHM}=5 \mathrm{~mm}$ and the RMSE for the $34 \mathrm{~mm}$ sphere. .43

Figure 23: (A) Phantom CT scan and SPECT images for iteration numbers 0, 6 and 15 left to right. (B) Plot of $\mathrm{Q}_{\mathrm{H}}$ (right axis) and RMSE (left axis) vs. iteration number for the $34 \mathrm{~mm}$ sphere (upper) and $\mathrm{Q}_{\mathrm{H}}$ for the $12 \mathrm{~mm}$ sphere peaking at different iteration number (bottom). ..... 44

Figure 24: Result of spatial resolution recovery showing the before and after ${ }^{90} \mathrm{Y}$ microsphere bremsstrahlung SPECT images of patients with the respective CT scans. (B) Line profiles through the $16 \mathrm{~mm}$ (lower) and $12 \mathrm{~mm}$ (upper right) spheres of the phantom ${ }^{90} \mathrm{Y}$ bremsstrahlung SPECT/CT image. SPECT images were resized to 512x512 for display purpose hence higher pixel position for the line profiles.

Figure 25: (upper) $\mathrm{Q}_{\mathrm{H}}$ and RMSE with $5 \mathrm{~mm}$ FWHM for the 34-mm sphere by HEGP collimator SPECT/CT imaging and (lower) the before (left) and after images at the fifth (middle) and fifteenth (right) iteration.

Figure 26: Result of $\mathrm{Q}_{\mathrm{H}}$ for varying FWHM and collimator at different iteration.

Figure 27: $\mathrm{Q}_{\mathrm{H}}$ (left axis) and RMSE (right axis) with $5 \mathrm{~mm}$ (left) and 6mm (right) FWHM for the $34 \mathrm{~mm}$ sphere by PET/CT TOF imaging of 15 (upper) and 30 (lower) minute imaging.

Figure 28: (left) Rod phantom and (right) IEC body phantom used in calibration factor study $(68)$

Figure 29: Anatomical representation (transverse, coronal and sagittal view) of method 1 (upper) and method 2 (lower) of calculating the CFs for the patient study.

Figure 30: SPECT/CT imaging window of ${ }^{99 \mathrm{~m}} \mathrm{Tc}$ (left), and line source filled with ${ }^{99 \mathrm{~m}} \mathrm{Tc}$ solution in air (middle) and water media (right).

Figure 31:Linear regression plots of the calibration curves with the 95\% CI range (green dashed lines) for method 1 (left) and method 2 (right).

Figure 32: Variation in the number of slices and voxel sized between the CT and SPECT slices between the pre and post-treatment images. 
Figure 33: Registration via changing the fixed image: (A) CT of pre-treatment, (B) CT of posttreatment and (C) SPECT of post-treatment.

Figure 34: Scatter plot of ${ }^{90} \mathrm{Y}$ microsphere SPEC images for patient 10 (left) and 13 (right) with administered activity of 1061.90 and $1235.8 \mathrm{MBq}$ respectively.

Figure 35: The iterative CV active contour segmentation method for different iteration number. 66

Figure 36: Patient 9 (left) and 13 (right) co-registered CT and SPECT images of ${ }^{99 \mathrm{~m} T c-M A A}$ (blue) and ${ }^{90} \mathrm{Y}$ (red) for reference images of CT of post-treatment (row A), CT of diagnostic (row B) and ${ }^{90} \mathrm{Y}$ microsphere SPECT (row C). The arrows in (B) show the misalignment between the CT and SPECT images showing activity distributions outside of the liver boundary in the coronal and sagittal views.

Figure 37: Plot of the probability distribution of the pre and post-treatment (upper) images and their joint distribution after co-registration.

Figure 38: (A) Mutual information between co-registered ${ }^{90} \mathrm{Y}$ microsphere and ${ }^{99 \mathrm{~m}} \mathrm{Tc}-\mathrm{MAA}$ SPECT images. (B) Patient $11 \mathrm{CT}$ (left), ${ }^{99 \mathrm{~m} T c-M A A}$ (middle) and ${ }^{90} \mathrm{Y}$ microsphere (right) SPECT scans showing a minimal uptake within the liver.

Figure 39: Patient 6 CT (left), ${ }^{99} \mathrm{~m}$ Tc-MAA (middle) and ${ }^{90} \mathrm{Y}$ microsphere (right) SPECT that showed the smallest mutual information between the co-registered pre and post-treatment images.

Figure 40: Plot of the segmented tumor volumes at each iteration for determining optimal iteration number $(n)$ at the convergence $(\varepsilon)$ calculated between consecutive volumes $\left(v_{n}\right.$ and

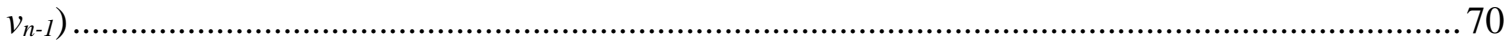

Figure 41: Segmented tumor in axial and coronal view. ..................................................... 70

Figure 42: Box plot of whole liver and tumor segmented volumes.......................................... 71

Figure 43: Scatterplot illustrating correlation between mean TLRs in 90Y and 99mTc-MAA SPECT.

Figure 44: Comparison of ${ }^{99 \mathrm{~m}} \mathrm{Tc}-\mathrm{MAA}$ SPECT/CT uptake distribution between patient 2 (left) and patient 8 (right).

Figure 45: Human body models of older (left) and newer version (right) for standard organ sizes $(81)$

Figure 46: Illustration of voxel dosimetry .78 
Figure 47: Plots of absorbed dose fraction for electrons (left) and photons of a $6 \mathrm{~mm}$ voxel on edge (78).

Figure 48: Display of details provided by DVH (A) and its implementation from activity and dose map images (B) where this implementation isn't possible for the MIRD method highlighted.

Figure 49: $\mathrm{S}$ values for various voxel sizes and interpolated results for $4.664 \mathrm{~mm}$ voxel size at different source coordinates.

Figure 50: Calculated voxel S values for ${ }^{90} \mathrm{Y}$ (left) and ${ }^{99 \mathrm{~m}} \mathrm{Tc}$ (right) within the beta range.

Figure 51: Bar plots of tumor (upper) and liver (lower) mean absorbed doses calculated by the three methods.

Figure 52: Box-Whisker plots of the calculated dose distribution in the tumor (left) and liver (volumes).

Figure 53: cDVH of tumor (A), fused isodose lines and CT scan for patient 3 (B) and cDVH of liver VOIs for all patients(C).

Figure 54: Fused isodose curves with the SPECT/CT of ${ }^{99 m}$ Tc-MAA (left) and ${ }^{90} \mathrm{Y}$ microsphere (right)

Figure 55: Dose distribution between ${ }^{90} \mathrm{Y}$ microsphere images before and after correction for collimator detector effect (left) and difference in $\mathrm{cDVH}$ (right).

Figure 56: Comparison of dose distribution calculated using developed and the MC method on

${ }^{90} \mathrm{Y}$ microsphere SPECT/CT images (left) and the resulting difference in $\mathrm{cDVH}$ (right). 87

Figure 57: Correlation of administered activity with healthy liver and tumor absorbed doses. .... 88 


\section{ABBREVIATIONS AND ACRONYMS}

2D

$3 \mathrm{D}$

${ }^{99 \mathrm{~m}} \mathrm{Tc}$

${ }^{90} \mathrm{Y}$

${ }^{18} \mathrm{~F}$

BSA

CDR

$\mathrm{CF}$

CNR

CT

DPK

FBP

FDG

FWHM

FOV

HEGP

LOR

LSF

MAA

MC

MEGP

MIRD

MLEM

OSEM

PET

PSF

$\mathrm{Q}_{\mathrm{H}}$
Two dimensional

Three dimensional

metastable Technetium-99

Yttrium-90

Fluoride-18

Body surface area

Collimator-detector response

Calibration factor

Contrast to noise ratio

Computed Tomography

Dose point kernel

Filtered back-projection

Fluorodeoxyglucose

Full width half maximum

Field of view

High energy general purpose

Line of response

Lung Shunt Fraction

Macroaggregated Albumin

Monte Carlo

Medium energy general purpose

Medical internal radiation dose

Maximum likelihood expectation-maximization

Ordered subset expectation-maximization

Positron Emission Tomography

Point spread function

Contrast recovery coefficient 


$\begin{array}{ll}\text { RMSE } & \text { Root mean square error } \\ \text { RMT } & \text { Radiomicroshpere Therapy } \\ \text { ROI } & \text { Region of interest } \\ \text { SPECT } & \text { Single Photon Emission Tomography } \\ \text { SPM } & \text { Statistical parametric mapping } \\ \text { TLR } & \text { Tumor to liver ratio } \\ \text { TOF } & \text { Time of flight } \\ \text { VOI } & \text { Volume of interest }\end{array}$




\section{Chapter 1: Introduction}

Radiomicrosphere therapy (RMT) using Yttrium-90 $\left({ }^{90} \mathrm{Y}\right)$ microspheres is an effective liver-directed therapy for treating primary or metastatic liver cancer $(1,2)$. The general procedure and the schematic of the steps are shown in Fig. 1 (I) and (II). The technique used in RMT entails the delivery of ${ }^{90} \mathrm{Y}$ microspheres into the hepatic artery to obtain a degree of selective uptake into the hepatic tumors by virtue of their predominant hepatic arterial supply, as opposed to the predominant portal venous supply to the normal liver as reported previously (3). ${ }^{90} \mathrm{Y}$ has a relatively long $\beta$ range $($ mean $=2.5 \mathrm{~mm}, \max =12 \mathrm{~mm})$ with minor irradiation of small tumors as these tumors won't be able to absorb all the emitted electrons, but irradiates more uniformly larger tumors ( $\geq 34 \mathrm{~mm}$ ) that often display heterogeneous perfusion. There are currently two types of commercially available ${ }^{90} \mathrm{Y}$ microspheres used in RMT: microspheres made of glass (TheraSphere) and resin (SIR-Spheres). In the glass microspheres, ${ }^{90} \mathrm{Y}$ is an integral constituent of the insoluble and non-biodegradable glass microspheres with diameter of each microsphere $20-30 \mu \mathrm{m}$. The resin microspheres have diameter $20-40 \mu \mathrm{m}$ where ${ }^{90} \mathrm{Y}$ is incorporated into the resin matrix where it is immobilized to the microspheres $(4) .{ }^{90} \mathrm{Y}$ microspheres are not metabolized, degraded or excreted but remain in the organ as a permanent implant (2).

In RMT, delivery of the radioisotope containing beads or microsphere is not meant to be occlusive to the arteries, unlike chemoembolization where the purpose is both to deliver high concentration of chemotherapy as well as slow (or stop) arterial flow to malignant tissues. The primary goal of RMT treatment is killing of tumors due to radiation; ischemia from occlusion of the artery is not desired since radiation therapy is less effective in hypoxic tissues, thus hepatic arterial flow in RMT is maintained (3). As a result, patient candidates for RMT treatment can also be those thought to be poor candidates for chemoembolization due to the fact that they are at high risk of mortality owing to acute liver necrosis from combined portal vein thrombosis and hepatic 
arterial occlusion. The biodistribution of ${ }^{90} \mathrm{Y}$ after treatment is currently assessed through ${ }^{90} \mathrm{Y}$ bremsstrahlung SPECT/CT imaging (5-7).
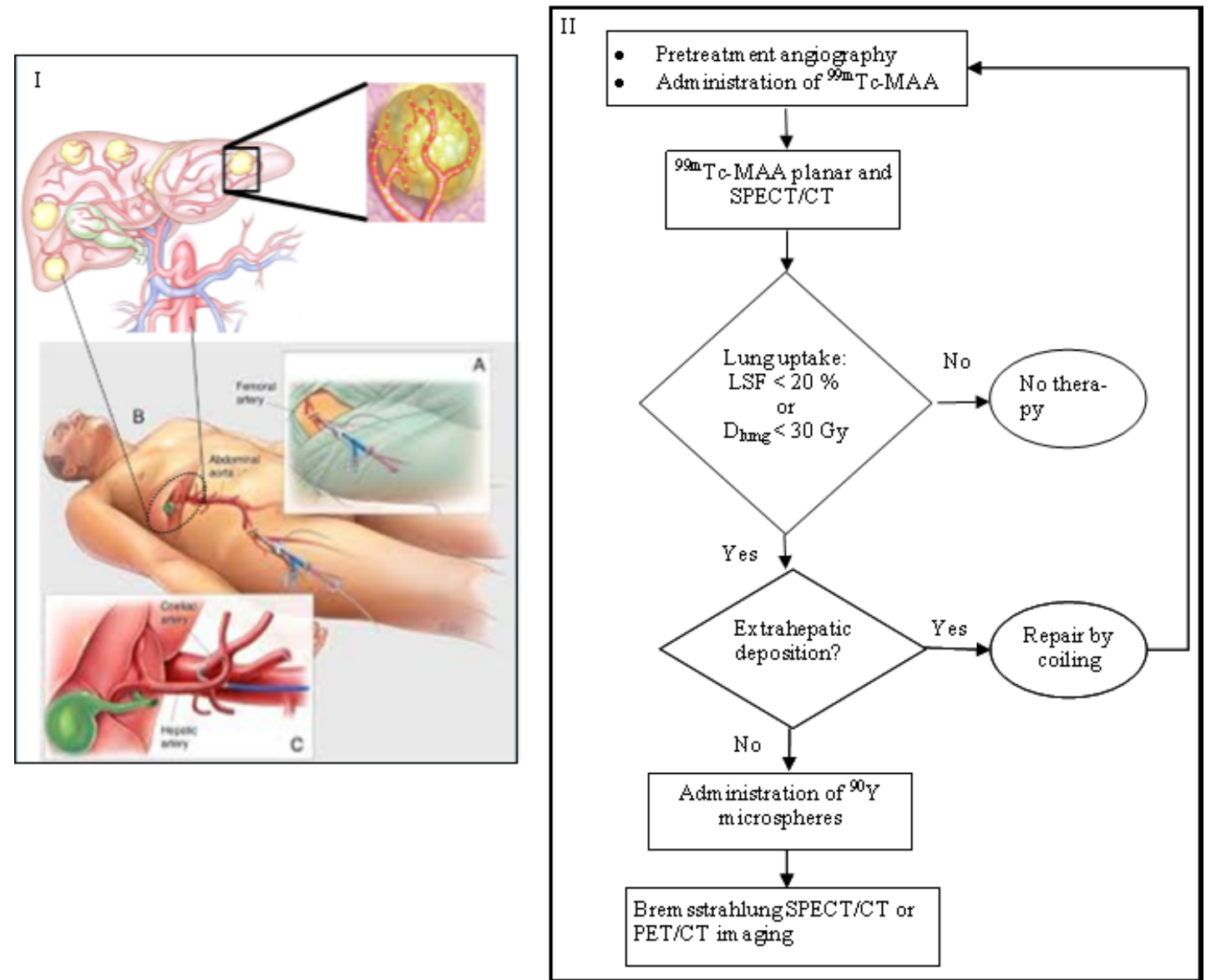

Figure 1:(I) RMT procedure: (A) introduction of a catheter into the femoral artery, (B) injection of microspheres, (C) liver vasculature (8). (II) Process diagram of RMT.

Technetium-99m macroaggregated albumin ( ${ }^{99} \mathrm{~m}$ Tc-MAA) scanning is performed prior to RMT as a surrogate for ${ }^{90} \mathrm{Y}$ microspheres. In pre-RMT planning, ${ }^{99 m} \mathrm{Tc}-\mathrm{MAA}$ planar and SPECT/CT imaging is used to measure the percentage of particles that shunt to the lungs (lung shunt fraction, LSF), to assess any extrahepatic particle deposition, and to calculate the tumor to normal liver ratio $(1,9)$. However, due to the significant differences in size and shape of the MAA 
and the microspheres ( ${ }^{99 \mathrm{~m}} \mathrm{Tc}-\mathrm{MAA}: ~ 10-50 \mu \mathrm{m}$ and ${ }^{90} \mathrm{Y}$ microspheres: $20-40 \mu \mathrm{m}$ ), and the position of the catheter tip between the ${ }^{99 \mathrm{~m}} \mathrm{Tc}-\mathrm{MAA}$ and ${ }^{90} \mathrm{Y}$ microsphere procedures, differences in the distribution between the two procedures may result. Multiple authors have demonstrated discrepancies of uptake and distribution between ${ }^{99 \mathrm{~m}} \mathrm{Tc}-\mathrm{MAA}$ and ${ }^{90} \mathrm{Y}$ microsphere SPECT/CT, questioning the predictive accuracy of ${ }^{99 \mathrm{~m}} \mathrm{Tc}-\mathrm{MAA}$ for $\operatorname{RMT}(9,10)$.
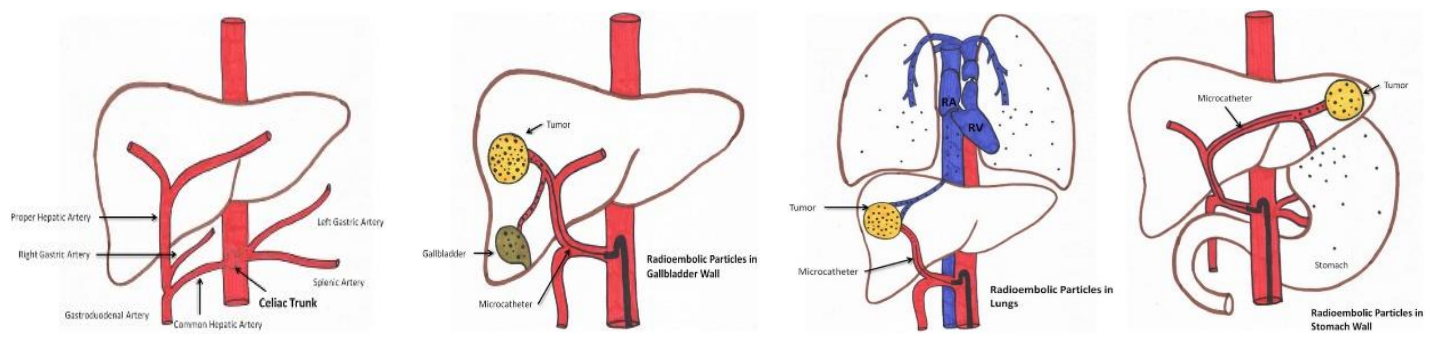

Figure 2: Various positions of a catheter tip during pretreatment stage and associated with unintended distribution (11).

Post RMT imaging of the ${ }^{90} \mathrm{Y}$ could potentially identify extrahepatic uptake. An early detection of such uptake, thus, could lead to the initiation of preventative measures early on (6). The tumor and liver dose estimates obtained from ${ }^{90} \mathrm{Y}$ microsphere bremsstrahlung SPECT/CT or PET/CT imaging could be correlated with tumor response and liver toxicity clinical data. The major problem with ${ }^{90} \mathrm{Y}$ bremsstrahlung imaging is the lack of a pronounced photopeak energy due to the continuous and broad energy spectrum of bremsstrahlung photons resulting it poor image quality. As a result, various studies based on phantoms and Monte Carlo (MC) simulations have recommended appropriate energy windows in accordance with the collimator used (12-15). It has been shown, often with phantom studies, the incorporation of MC simulation to clinical images can provide optimally accurate bremsstrahlung images by compensating for attenuated and scattered photons through correction for attenuation scatter and collimator-detector response (14-16). Alternately, studies have demonstrated the feasibility of ${ }^{90} \mathrm{Y}$ microsphere PET/CT imaging excelling in contrast and resolution compared to bremsstrahlung $\operatorname{SPECT/CT}(17,18)$. A recent study 
by Yue et al. (18) concluded that both modalities are comparable for post-treatment dosimetry estimation if appropriate reconstruction for ${ }^{90} \mathrm{Y}$ bremsstrahlung SPECT/CT is applied. However, an appropriate reconstruction method requires incorporation of $\mathrm{MC}$ simulation in ${ }^{90} \mathrm{Y}$ bremsstrahlung SPECT/CT for quantitative improvement. But the MC based approach is not commercially available and is not easily applied for clinical implementation (19,20). Siman el al. (19) recently developed a practical imaging protocol employing background compensation for ${ }^{90} \mathrm{Y}$ microsphere SPECT/CT imaging as an alternative to the MC method. The authors assert that their method doesn't address the main image degrading factors in ${ }^{90} \mathrm{Y}$ bremsstrahlung SPECT imaging, such as object scatter, septal penetration, and backscatter; nonetheless, they stated an improvement in recovery coefficient from $39 \%$ to $90 \%$ in a $37 \mathrm{~mm}$ sphere in a $10 \mathrm{~mm}$ volume of interest.

The present dissertation addresses the development and application of post-reconstruction techniques to improve the quality and quantitation of ${ }^{90} \mathrm{Y}$ SPECT/CT and PET/CT images. Different imaging windows, collimators, acquisition times and reconstruction algorithms were investigated with a phantom study. Issues related to calibration factor for activity estimation from patient and phantom ${ }^{90} \mathrm{Y}$ SPECT/CT images as well as PET/CT images were addressed. Co-

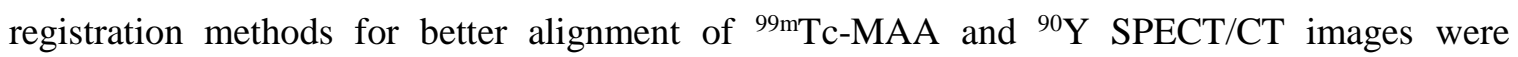
identified and evaluated. Subsequently, the correlation of the uptake pattern on the improved ${ }^{90} \mathrm{Y}$ microsphere SPECT post-therapy images and on pre-treatment ${ }^{99 m}$ Tc-MAA SPECT images was evaluated. A three-dimensional (3D) absorbed dose estimation (dosimetry) algorithm using the voxel S-value method was developed. Thus, the association between administered activity and liver and tumor dosimetry in the patient study was analyzed.

\subsection{Objective, specific aims and hypotheses}

The overall objective is to achieve a quantitative improvement of ${ }^{90} \mathrm{Y}$ bremsstrahlung SPECT and PET images and a subsequent comparison of uptake pattern between pretreatment ${ }^{99 \mathrm{~m}} \mathrm{Tc}-\mathrm{MAA}$ and 
improved ${ }^{90} \mathrm{Y}$ bremsstrahlung SPECT images, and finally to develop a 3D dosimetry method for estimation of ${ }^{90} \mathrm{Y}$ absorbed dose in tumor and healthy liver.

This objective was accomplished by addressing the following specific aims:

\subsubsection{Specific Aim 1}

Improve the quantitative quality of ${ }^{90} \mathrm{Y}$ bremsstrahlung SPECT and PET images through evaluation of various imaging energy windows, collimator types, acquisition times, reconstruction algorithms and the development of a post-reconstruction image improvement algorithm.

The outcome of this aim provided improved images for the subsequent analyses. This aim included estimation of the camera point spread function (PSF).

\subsubsection{Specific Aim 2}

Compare the uptake pattern in pre-treatment ${ }^{99 \mathrm{~m}} \mathrm{Tc}-\mathrm{MAA}$ and post-treatment ${ }^{90} \mathrm{Y}$ microsphere SPECT/CT images.

The outcome of this aim was to assess the degree of accuracy by which ${ }^{99 \mathrm{~m}} \mathrm{Tc}-\mathrm{MAA}$ SPEC/CT can predict treatment outcome. This aim includes co-registration of ${ }^{99 \mathrm{~m} T c-M A A}$ and ${ }^{90} \mathrm{Y}$ microsphere SPECT/CT images.

\subsubsection{Specific Aim 3}

Develop an algorithm using the voxel $\mathrm{S}$ value method to transform ${ }^{90} \mathrm{Y}$ bremsstrahlung SPECT and PET image pixel values (counts per second, cps) to absorbed dose values (Gy) and generate absorbed dose rate distribution at the voxel level.

The outcome of this aim was to generate images with absorbed dose values at the voxel level. This aim includes calculation of the camera calibration factor (sensitivity). This aim also includes analysis of the correlation between administered activity and absorbed doses in tumorous and healthy liver. 


\section{Chapter 2: Background}

2.2 Nuclear Medicine Imaging Modalities in Liver Cancer Management

There are various imaging modalities used in the diagnosis, treatment planning and evaluation of liver cancer: ultrasound, MRI, CT, planar nuclear imaging, SPECT and PET. Among which planar, SPECT and PET fall under nuclear medicine imaging, which the dissertation will be focusing on. CT, typically used as a diagnostic tool, is also incorporated into the dual imaging modalities, SPECT/CT and PET/CT, and used for correcting image degrading factors. Thus, it is discussed in conjunction with the dual modalities.

\subsubsection{Gamma/ planar imaging}

The Anger camera, named for its inventor Hal O Anger, or gamma/planar/scintillation camera is the most commonly used static imaging device in nuclear medicine, and was commercialized as early as mid-1960's (21). The major components of a gamma camera are a collimator, a large area $\mathrm{NaI}(\mathrm{TI})$ scintillation crystal, a light guide, and an array of photomultiplier tubes (PMT). The basic principle how the camera works is shown in Fig 3. The collimator consists of a very large lead plate with many small holes through it. Only those photons that are perpendicular to the collimator plane (or parallel to the axes of the holes) can pass cleanly through the holes to be absorbed by the scintillation crystal. There are different kinds of collimators used in clinical practice, depending on the type of radionuclide imaged (Table 1). The scintillation crystal is commonly made from sodium iodide with a small amount of thallium impurity, NaI(TI). The crystal emits light photons whenever it absorbs a gamma ray, where the intensity of the light is directly proportional to the energy of the gamma ray. The purpose of the PMT is to act as a transducer by changing the visible light from the crystal to electric current, which is directly proportional to the amount of light. The current pulses then go through a preamplifier before being sent for further processing. The outputs of the PMT are converted into a three-dimensional signal after processing. Two of the signals ( $\mathrm{X}$ and $\mathrm{Y}$ ) contain the spatial location of the scintillation while 
the energy deposited in the crystal is represented in $\mathrm{Z}$ to portray a 3D distribution of a radioactive decay as a 2D image with no depth information. Due to this and the selectivity of the collimator in passing gamma rays, the resolution of a gamma camera is between $6.4-12.6 \mathrm{~mm}$, with considerable loss of contrast and sensitivity (0.1\%, so that $99.9 \%$ photons are not detected) (22). The preferred emission energies of gamma $(\gamma)$ rays ranges from 80 to $500 \mathrm{keV}$ with $20-25 \%$ window width from the photopeak $(21,23)$. Gamma rays of these energies can sufficiently penetrate body tissues to be detected from internal organs, and can be stopped efficiently by dense scintillators.

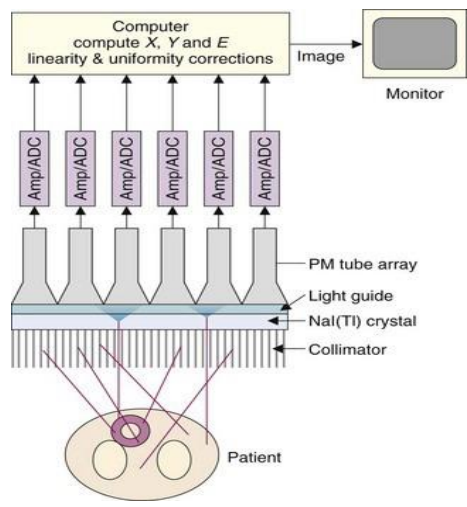

Figure 3: Basic components of gamma camera (23).

Table 1: Collimator specification at $10 \mathrm{~cm}$ from collimator face (22).

\begin{tabular}{ccccccc}
\hline Collimator type & $\begin{array}{c}\text { Hole } \\
\text { Diameter } \\
(\mathrm{mm})\end{array}$ & $\begin{array}{c}\text { Septal } \\
\text { Thickness } \\
(\mathrm{mm})\end{array}$ & $\begin{array}{c}\text { Hole } \\
\text { Length } \\
(\mathrm{mm})\end{array}$ & $\begin{array}{c}\text { Resolution } \\
(\mathrm{mm})\end{array}$ & $\begin{array}{c}\text { Sensitivity } \\
(\mathrm{cps} / \mathrm{MBq})\end{array}$ & $\begin{array}{c}\text { Energy } \\
(\mathrm{keV})\end{array}$ \\
\hline $\begin{array}{c}\text { Low-energy high- } \\
\text { resolution (LEHR) } \\
\begin{array}{c}\text { Low-energy } \\
\text { general purpose } \\
\text { (LEGP) }\end{array}\end{array}$ & 1.4 & 0.152 & 32.8 & 6.4 & 91 & 140 \\
$\begin{array}{c}\text { Medium-energy } \\
\text { general purpose } \\
\text { (MEGP) }\end{array}$ & 2.95 & 0.180 & 24.7 & 8.3 & 149 & 140 \\
$\begin{array}{c}\text { High-energy } \\
\text { general purpose } \\
\text { (HEGP) }\end{array}$ & 3.81 & 1.727 & 60.0 & 12.6 & 61 & 360 \\
\hline
\end{tabular}


In liver cancer management, planar imaging (Fig. 4) is used in the 'mapping' phase where diagnostic angiography and intra-arterial administration of ${ }^{99 \mathrm{~m} T c-M A A}$ microspheres is performed that mimic the distribution of ${ }^{90} \mathrm{Y}$ microspheres. Diagnostic angiography is used to identify arteries arising from the hepatic artery to determine if coil embolization is necessary and to select the best catheter position for tumor targeting. ${ }^{99 \mathrm{~m}} \mathrm{Tc}-\mathrm{MAA}$ planar imaging is done for determining the percentage of particles that shunt to the lungs (lung shunt fraction, LSF) which might require adjustment of the amount of ${ }^{90} \mathrm{Y}$ microsphere administered to reduce potential damage to the lungs.

$$
L S F=\frac{\text { Lung counts }}{\text { Lung counts }+ \text { liver counts }}
$$

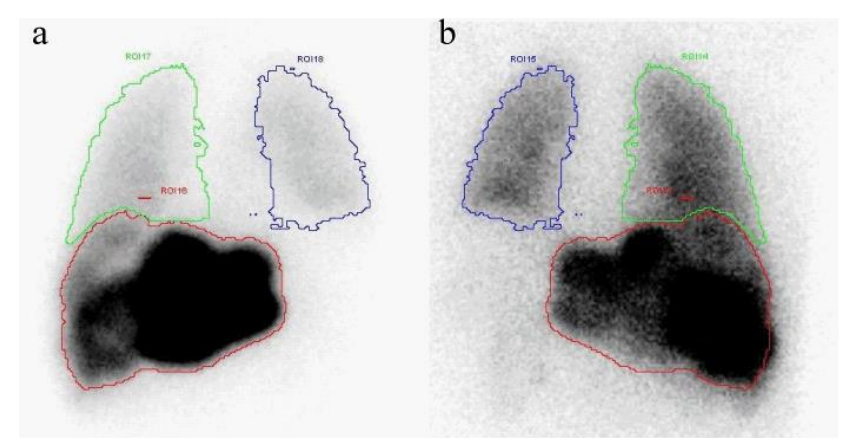

Figure 4: Example of planar images with ROIs drawn over the anterior (a) and posterior (b) of lungs and liver images to determine the LSF (24).

\subsubsection{Single photon emission tomography (SPECT)}

Nowadays gamma cameras with SPECT capability of dual-head detectors is employed in most clinical centers. SPECT imaging overcomes the drawbacks of planar imaging by providing 3D information with depth information by collecting views from different directions. The total rotation of a SPECT gantry around a patient is usually $180^{\circ}$ or $360^{\circ}$, and the total number of projections may vary from 64 to 128 . Although data acquired over an $\operatorname{arc}$ of $180^{\circ}$ are sufficient for tomographic reconstruction in SPECT, there are advantages in terms of resolution uniformity and correction for $\gamma$-rays attenuation in acquiring data over a full 360 degree arc. The acquisition pixel 
matrix should be of the same order as the number of projections to avoid the appearance of artifacts, thus clinical SPECT images are reconstructed in a matrix of $64 \times 64$ or $128 \times 128$ pixels. Cross sectional images are produced for all axial locations (slices) covered by the field of view (FOV) of the gamma camera, resulting in a stack of contiguous $2 \mathrm{D}$ images that form a $3 \mathrm{D}$ image volume. The common methods to reconstruct 3D images from planar images are the filtered back-projection (FBP) and iterative methods such as maximum likelihood expectation maximization (MEM). Mathematical filters are used to obtain an image of adequate quality but since filters change raw images, the choice of a particular filter and its parameters depends on the physical characteristics of the organ under study. Several types of artifacts may appear using the SPECT techniques that are related to instrumentation, patient, study acquisition or data processing.

A SPECT/CT system consists of a single unit that integrates SPECT and CT (Fig. 5), allowing data acquisition of each modality in a single patient study. The CT images are used both for attenuation correction and for anatomical location. When CT is used for SPECT attenuation correction, the resolution of CT data is reduced to match the SPECT. In addition, since the effective energy of the $\mathrm{x}$-ray beam is about $70 \mathrm{keV}$ and the attenuation varies with energy, the $\mathrm{CT}$ attenuation map is converted to the radionuclide photon energy used in SPECT (for example, about 140keV when ${ }^{99 m} \mathrm{Tc}$ is used). The total time of a SPECT/CT study is significantly reduced because of the fast acquisition speed of CT compared to SPECT. The anatomical images acquired with CT can be merged with the SPECT emission images to provide functional anatomical maps for precise localization of radiotracer uptake. The hybrid technique allows precise localization of primary tumors and evaluation of extent of disease. In hybrid SPECT/CT, SPECT is performed in a $360^{\circ}$ rotation, consisting of 60 projections $6^{\circ}$ apart in a matrix of $128 \times 128$ (25). 


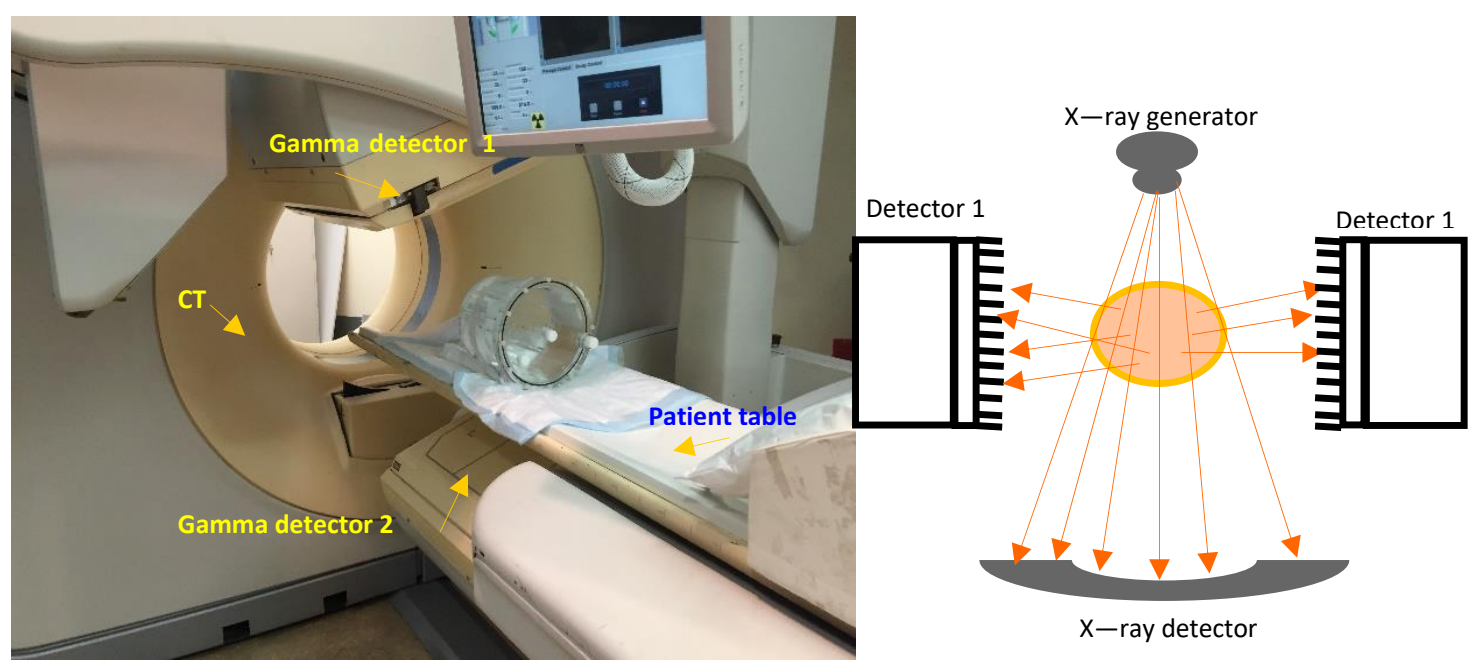

Figure 5: SPECT/CT camera (left) and its schematic (right).

The fundamental relationship between the projection space and the image (object) space in SPECT image formation (Fig. 6) is given as:

$$
p(s, \theta)=\iint f(x, y) \delta(x \cos \theta+y \sin \theta-s) d x d y
$$

Where $f(x, y)$ is object representation in image space, $\delta(.$.$) is the Dirac delta function, and g(r, \theta)$ is the projection data along $\theta$ and $r$ of projection angle and line respectively. In SPECT however, this relationship is not linear due to image degrading factors discussed below.
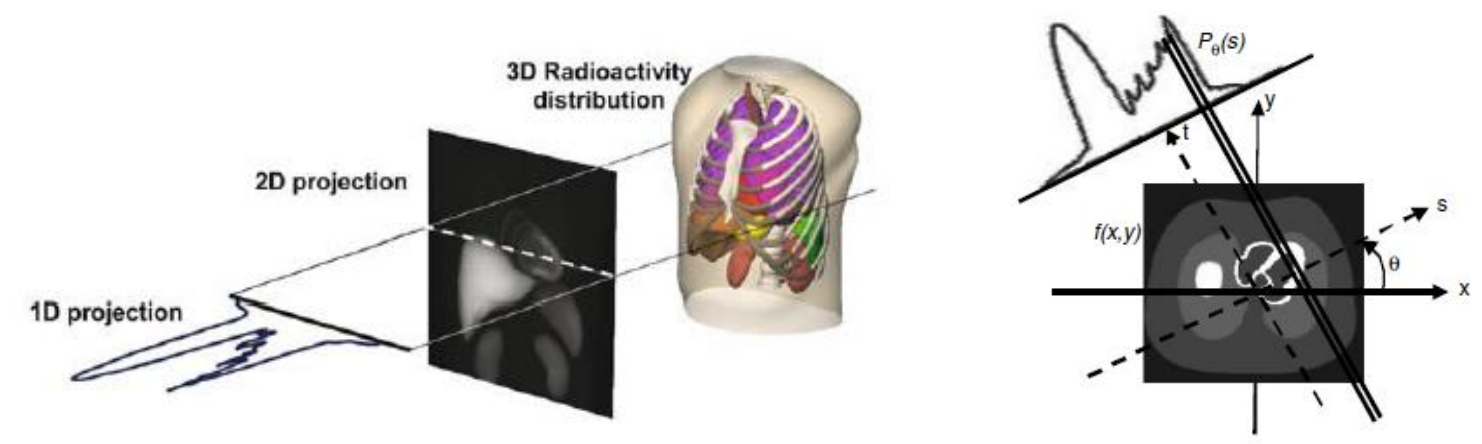

Figure 6: SPECT image formation (left) and 2D image reconstruction (right) (26). 
Image degrading factors in SPECT

\section{Photon attenuation and scatter}

There are five types of $\mathrm{x}$ - and $\gamma$-ray interactions with matter: Compton scattering, photoelectric effect, pair production, Rayleigh (coherent) scattering and photonuclear interactions. The first three interactions are the most important as they result in the transfer of energy to electrons which then impart the energy to matter. But the importance of these interactions depends both on the photon quantum energy $\left(\mathrm{E}_{\gamma}=h v\right)$ and the atomic number $\mathrm{Z}$ of the absorbing medium (Fig 7). Rayleigh scattering is elastic scattering where the photon is redirected with no energy loss and photonuclear interactions are only significant for high energy photons $(\mathrm{E}>>\mathrm{MeV})$ where there is production of neutrons. The major interaction that occur in SPECT imaging are photoelectric and Compton scattering due to the range of photon energies detected $(80-500 \mathrm{keV})$. Photoelectric effect is dominant at lower energies and mostly occurs in high $\mathrm{Z}$ media such as lead while the Compton scattering occurs at medium energy and is dominant in low $\mathrm{Z}$ media such as human tissue, water, etc. Each of these effects contribute to photon attenuation and scatter in SPECT. Attenuation results from absorption by the photoelectric effect, Compton scattering, and pair production at a higher energy depending on the photon energy and thickness of the absorber (Eqn. 3). Scattering in SPECT is mainly due to Compton scatter where the photon is deflected at an angle $\Theta\left(0-180^{\circ}\right)$ from its original direction with a reduced energy due to its interaction with an outer shell electron of the absorber atom (Eqn. 4). For $\Theta=180^{\circ}$, backscattering collision results.

$$
\begin{gathered}
I_{t}=\sqrt{I_{1} \times I_{2}}=\sqrt{\left[\left(\llbracket I_{01} \times I_{02}\right) e \rrbracket^{-\mu(a+b)}\right.}=\sqrt{I_{01} \times I_{02}} e^{-\mu(D / 2)} \\
E_{S c}=\frac{E_{0}}{\left[1+\frac{E_{0}}{m c^{2}}(1-\cos \theta)\right]}
\end{gathered}
$$


$I_{01}$ and $I_{02}$ are the initial photons, $a$ and $b$ are source depths, $I_{t}$ is the total attenuated photons transmitted from the two detectors, $\mu$ is the linear attenuation coefficient and $D=a+b$, thickness of the absorber, $E_{s c}$ and $E_{0}$ are the scattered and initial photon energies respectively.

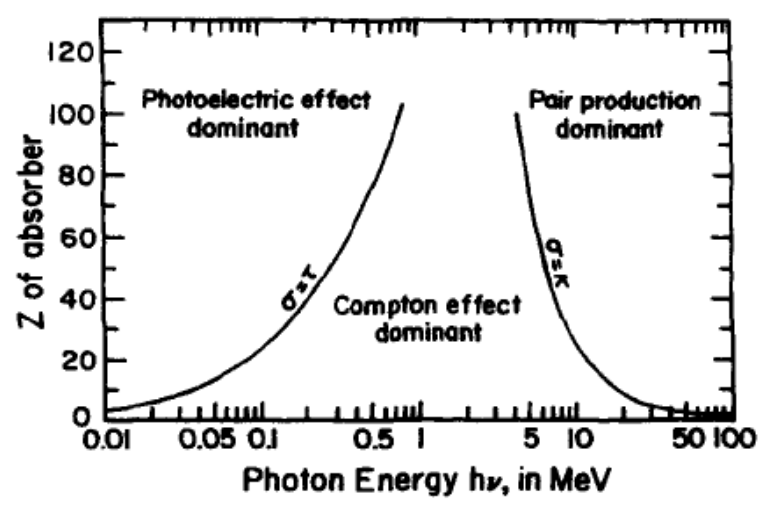

Figure 7: The three major types of photon interactions in SPECT(27).

\section{Collimator detector response}

Collimator detector response (CDR) is the response of the camera from a point source activity where the shape of the response determines the resolution in SPECT $(28,29)$. CDR varies with distance from the detector (Fig. 8) causing different blurring at different distances but CDR is spatially invariant at a constant distance, $\mathrm{d}$, from the detector surface. CDR in SPECT has four components: intrinsic response, geometrical response, septal penetration and septal scatter. The last two components are particularly problematic for high energy emitters such ${ }^{90} \mathrm{Y}$ causing additional loss of image contrast due to degradation of spatial resolution. The intrinsic response is the response of the scintillation camera, excluding the collimator, to a pencil beam of radiation. Intrinsic response is dependent on the energy of the incident photon, the energy window used, and the thickness and composition of the crystal and it represents the efficiency of the crystal in detecting photons. The geometric response is the total collimator response function that represents the distribution of the detected photons that travel through the collimator hole without interacting or passing through the collimator septa and is the easiest to treat theoretically. The general form of 
the collimator-detector response function is given in Eqn. 5. Geometric collimator resolution $\left(\mathrm{R}_{\mathrm{c}}\right)$ is given by Eqn. 6 .

$$
\begin{gathered}
d(s, D)=i(s) \times\left(g_{c}(s, D)+p_{s}(s, D)+s_{s}(s, D)\right) \\
g_{c}=d \frac{\left(l_{e}+b\right)}{l_{e}}
\end{gathered}
$$

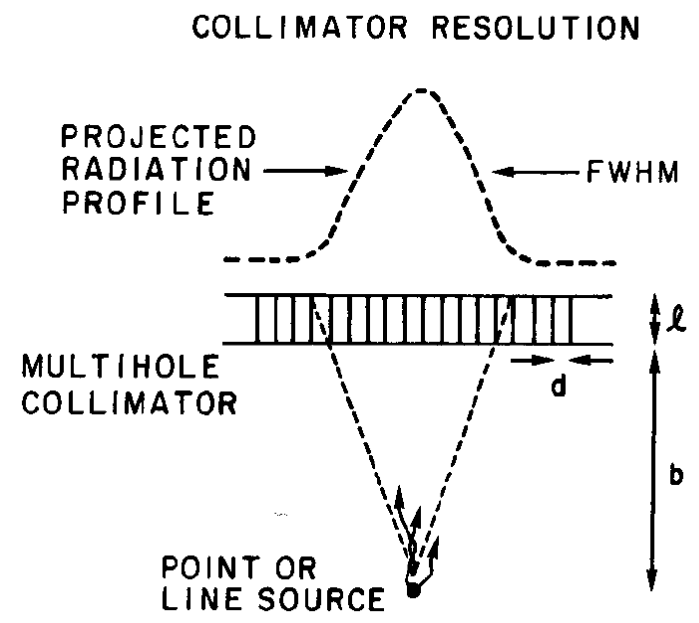

Figure 8: Profile of a parallel hole collimator for a point source (23).

Where $\mathrm{D}$ and $\mathrm{b}$ are the distance from the radiation source to the collimator, $\mathrm{i}(\mathrm{s})$ is the intrinsic point response function, $\mathrm{g}_{\mathrm{c}}(\mathrm{s}, \mathrm{D}), \mathrm{p}_{\mathrm{s}}(\mathrm{s}, \mathrm{D})$, and $\mathrm{s}_{\mathrm{s}}(\mathrm{t}, \mathrm{D})$ are the collimator geometric, septal penetration and septal scatter correction respectively, and $\mathrm{d}$ is the diameter and le $=1-2 \mu^{-1}$ the effective length of the collimator holes. For simplicity, the septal penetration and scatter are neglected giving the system resolution $\left(R_{s}\right)$ of SPECT camera as the combined effect of the intrinsic $\left(R_{i}\right)$ and geometric $\left(\mathrm{R}_{\mathrm{c}}\right)$ resolutions:

$$
R_{S}=\sqrt{R_{i}^{2}+R_{c}^{2}}
$$

As a result of these image degrading factors in SPECT, the projection data in the image formation given by Eqn. 8 will be modified to include these effects as follows:

$$
p(s, \theta)=\int_{-\infty}^{\infty} d t \int d s^{\prime} \times f\left(s^{\prime}, t\right) \times s f\left(s^{\prime}, t ; s\right) \times d f\left(s^{\prime}-s ; D+t\right) \times \exp \left[-\int_{t_{d}}^{t} \mu\left(s^{\prime}, t^{\prime}\right) d t^{\prime}\right]
$$


Where $s^{\prime}=\left(s_{u}, s_{v}\right), s f\left(s^{\prime}, t ; s\right)$ is the scatter response function, $d f$ is the collimator detector response, $D$ is the distance between the collimator and the center of rotation and $z=D+t$, and $t_{d}$ is the tcoordinate of the detection plane(26). The equation is further complicated by the inclusion of $3 \mathrm{D}$ collimator-detector response. The following step of image reconstruction is to find a solution of $f(s, t)$ from the projections described by Eqn. 8 .

\section{SPECT image reconstruction}

Image reconstruction is done using mathematical algorithms to reconstruct images from the multiple projections of detected emissions from radionuclides within a subject, known as emission computed tomography (Fig. 6). Although the instrumentation in CT, SPECT and PET differ, the mathematics of image reconstruction are the same. There are different techniques of image reconstruction in tomography: simple back-projection, direct Fourier Transform, filteredback-projection and iterative reconstruction algorithms. Iterative methods such as maximum likelihood expectation maximization (MLEM), particularly its accelerated version OSEM, offer potential benefits that lead to better results. The pronounced property of iterative reconstruction is that it can be modified to incorporate weights or penalties such as correction for photon attenuation, scatter and compensation for spatial resolution losses which enables the algorithm to be tuned for specific clinical requirements. The method is suggested for quantitative SPECT image analysis since it allows for optimal correction of image degrading physical effects, improving noise properties. In addition, compensation for physical limitations can be modelled in the reconstruction process of iterative methods(30). SPECT/CT imaging in liver cancer management is used at two stages: pretreatment ${ }^{99 \mathrm{~m}} \mathrm{Tc}-\mathrm{MAA}$ and post-treatment ${ }^{90} \mathrm{Y}$ imaging.

${ }^{99 m}$ Tc-MAA Imaging

Technetium-99m macroaggregated albumin ( ${ }^{99 \mathrm{~m} T c-M A A)}$ scanning using SPECT or SPECT/CT modality is performed before ${ }^{90} \mathrm{Y}$ RMT in order to detect activity outside of the liver 


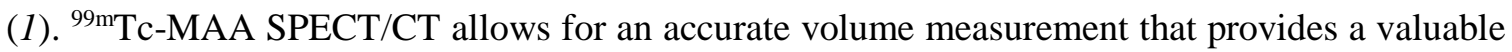
contribution to the therapeutic planning of patients with liver cancer. It can be used to determine liver volume, and for quantitation of injected dose. In pre-RMT planning, dosimetry based on ${ }^{99 m}$ Tc-MAA SPECT/CT can be used for selection of patients and assess any potential extrahepatic particle deposition. ${ }^{99 \mathrm{~m}} \mathrm{Tc}-\mathrm{MAA}$ SPECT/CT is also used to calculate the tumor to normal liver ratio (TLR) (9). Based on these determined values the administered ${ }^{90} \mathrm{Y}$ activity dose $\left(\mathrm{A}_{\text {inj }}\right)$ is calculated from a well-defined model [Fig. 9(A)] according to the Medical Internal Radiation Dose (MIRD) scheme, using the empirical method given by Eqn. 9 or by the body surface area (BSA) method given by Eqn. 11 (31). The absorbed dose in the tumor, healthy liver and lungs is calculated using Eqn. 12 where the fractional uptake is the fraction of the administered activity expected to be deposited within the volume of interest.

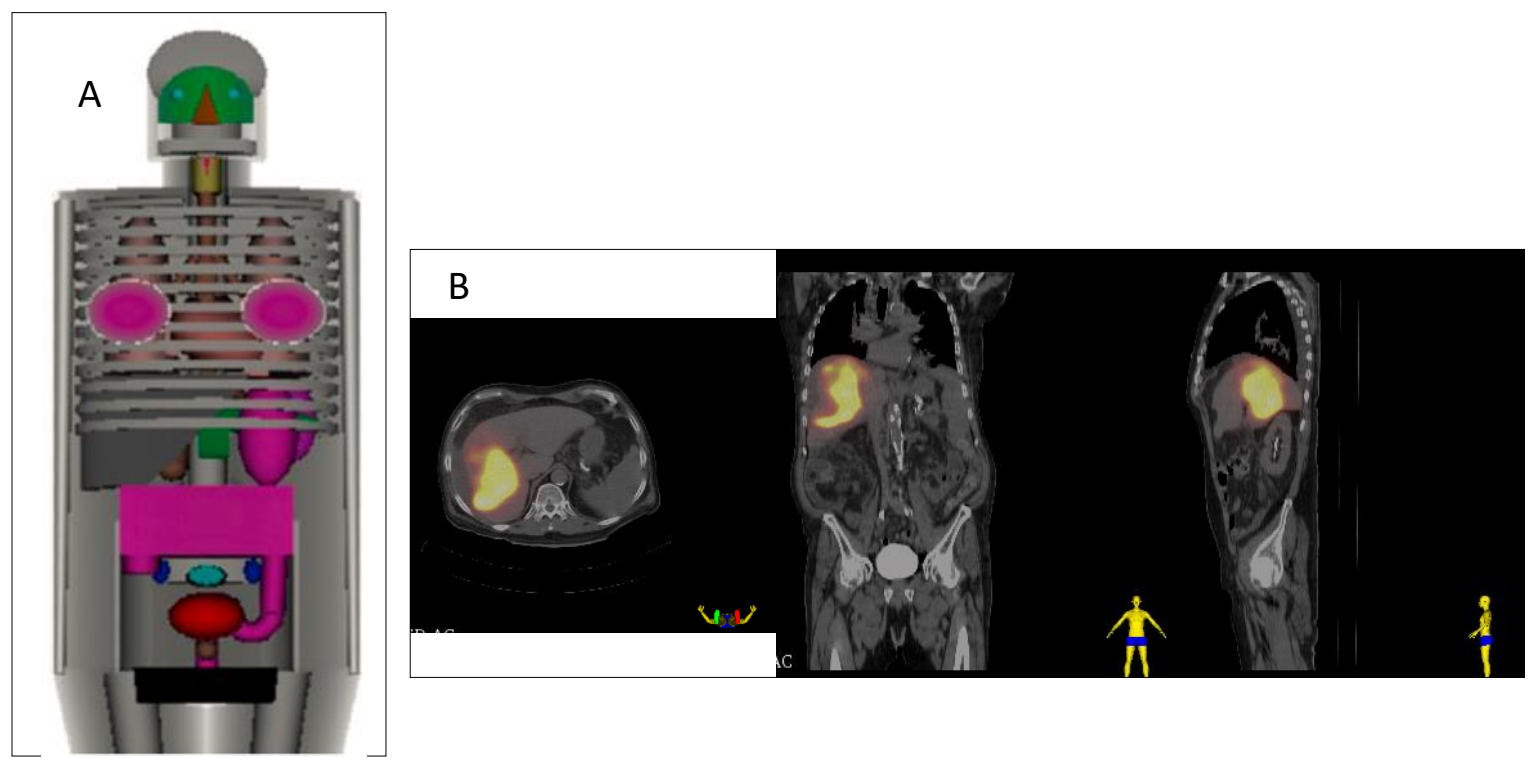

Figure 9: (A) Reference anatomic model used for conventional dose calculation (32) and (B) ${ }^{99 m}$ Tc-MAA SPECT/CT scan of a patient. 


$$
\text { Ainj }= \begin{cases}2 G B q, & \text { if } r<25 \% \\ 2.5 G B q, & \text { if } 25 \%<r<50 \% \\ 3 G B q, & \text { if } r>50 \%\end{cases}
$$

Where $r=(\text { Tumor volume/Total liver volume })^{*} 100$

$$
\begin{gathered}
\text { TLR }=\frac{\text { Max tumor counts }}{\text { Average liver counts }} \\
A_{\text {inj }}(G B q)=B S A-0.2+\frac{\text { Tumor volume }}{\text { Tumor volume }+ \text { liver volume }} \\
\text { Dose }(G y)=\frac{A_{\text {in } j \times 49,800 \times \text { fractional uptake }}}{\text { Mass }}
\end{gathered}
$$

Based on ${ }^{99 m}$ Tc-MAA SPECT/CT imaging patients with LSF higher than $20 \%$ or absorbed dose in the lungs $\geq 30 \mathrm{~Gy}$ for ${ }^{90} \mathrm{Y}$ resin and glass microspheres respectively are disqualified for RMT treatment (33).

\section{${ }^{90}$ Y SPECT Imaging}

Post-treatment ${ }^{90} \mathrm{Y}$ bremsstrahlung imaging is recommended by most physicians and is currently a routine imaging procedure performed during the first $24 \mathrm{hrs}$ of treatment $(5-7,34)$. The main reasons for post treatment imaging is to potentially identify the unexpected presence of ${ }^{90} \mathrm{Y}$ microspheres outside the liver which will likely cause serious complications such as ulceration and GI tract bleeding $(17,34)$. The number of ${ }^{90} \mathrm{Y}$ microspheres injected is very high compared to ${ }^{99 \mathrm{~m}} \mathrm{Tc}-$ MAA which might lead to a more powerful embolic effect that contributes to slowing down the blood flux or even induces transient blockade of the targeted vessels. This can significantly modify the arterial flow, resulting in a distribution of the ${ }^{90} \mathrm{Y}$ microspheres different to that of the ${ }^{99 \mathrm{~m}} \mathrm{Tc}-$ MAA (35). ${ }^{90} \mathrm{Y}$ accumulation in organs other than liver causes serious complication due to the high radiosensitivity of the surrounding organs. Thus early detection of extrahepatic activity will help in preventing development of more serious problems through aggressive pain management such as surgery, angioplasty or stent, supportive management, etc. based on the symptoms developed 
$(17,36)$. Dosimetry of tumorous and non-tumorous tissues from post-treatment imaging could be correlated with tumor response and liver toxicity. The major problem in bremsstrahlung SPECT imaging is the lack of pronounced photopeak energy due to the continuous and broad energy spectrum of bremsstrahlung photons (Fig. 10). The absence of a photopeak energy window makes it difficult to achieve window based scatter rejection, scatter correction, and attenuation correction which all contribute to low quality bremsstrahlung SPECT images (17). The difficulty to separate the counts on the image that come from primary photons, from those that have been scattered, penetrated the collimator septa, or back-scatter from the camera housing and photomultiplying tubes make it very complex to achieve scatter correction. At any energy window, the ratio of primary bremsstrahlung to the total number of photons detected is $<15 \%$, with the highest primary fraction occurring between $80-180 \mathrm{keV}$ (37-39). Despite the wide range of energies imaged during bremsstrahlung SPECT, it is agreed upon by authors to use a single effective attenuation coefficient for attenuation correction $(40,41)$. However, this would result in discrepancy of the actual value of the attenuation coefficient and the reconstruction technique used. It has been shown, often in conjunction with phantom studies, that Monte Carlo simulations help to optimize the accuracy of bremsstrahlung images by enabling correction for attenuation, scatter and collimatordetector response with error less than $11 \%$ for sphere volumes about $100 \mathrm{ml}$ and larger $(14,16,42)$. Proper attenuation correction requires an attenuation map specific to the object being imaged, patient specific in a clinical case. To achieve dosimetry based on the image at hand, i.e. patient specific dosimetry, applying attenuation correction on the image is one of the necessities (30). Photon attenuation correction is especially critical in the clinical setting where thickness of tissues varies for different regions of the patients' anatomy (26). 

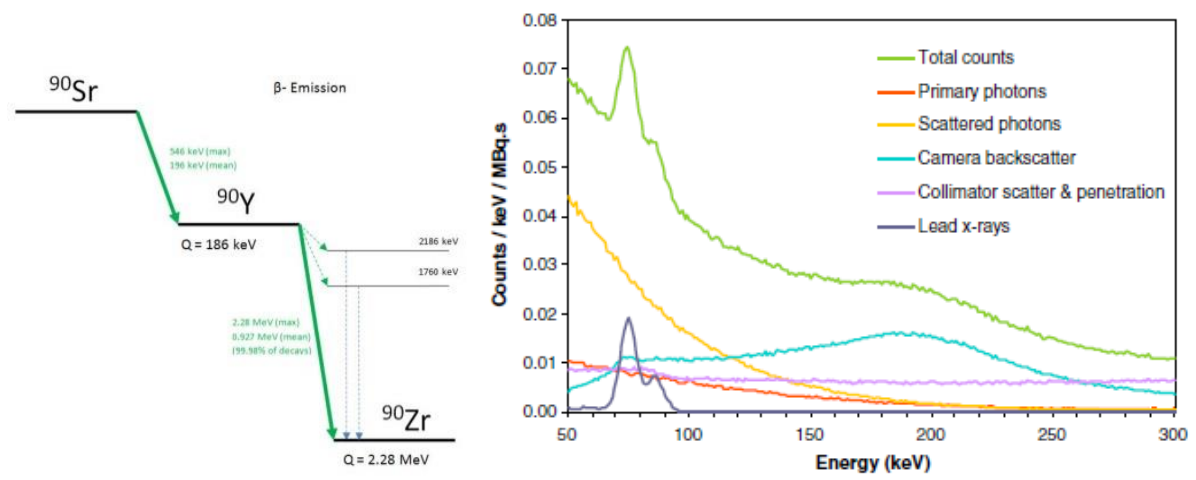

Figure 10: Schematic representation of the energy levels (43) and energy spectrum of ${ }^{90} \mathrm{Y}(7)$.

Determining an optimal energy window where these effects are minimized is critical for accurate dosimetry. As a result different acquisition energy windows have been recommended by many authors (12-15). In a phantom study and Monte Carlo simulation Minarik et al. (14) suggested that adequate image quality can be obtained in the $105-195 \mathrm{keV}$ energy window, and it was stated that a window with a lower limit below $\sim 80 \mathrm{keV}$ will include counts for characteristics X-ray photons produced in the lead collimator. Ito et al. (13) used three energy windows centered at $75 \mathrm{keV} \pm 25 \%(57-94 \mathrm{keV}), 120 \mathrm{keV} \pm 15 \%(102-138 \mathrm{keV})$ and $185 \mathrm{keV} \pm 25 \%(139-232 \mathrm{keV})$ in a phantom study. They found that bremsstrahlung SPECT/CT acquisition centered at the $120 \mathrm{keV}$ energy window resulted in the highest spatial resolution. A wide energy window $(55-285 \mathrm{keV})$ with medium-energy collimation was used by Shen et al. (12) to optimize sensitivity, but this window results in loss of spatial resolution or requires high-energy collimator and restoration filters (40). In clinical practice, image acquisition centered on $90 \mathrm{keV} \pm 15 \%$ is currently a standard protocol. In addition to choosing the optimal energy window for imaging bremsstrahlung photons, various pre and post reconstruction optimization efforts have been done to improve bremsstrahlung image quality. The same groups who studied the various acquisition windows have investigated various scatter correction techniques $(14,15)$. Ahmadzadehfar et al. (34) addressed the usefulness and significance of bremsstrahlung SPECT/CT imaging for post-treatment dosimetry. In the 
manuscript, it is shown that ${ }^{90} \mathrm{Y}$ bremsstrahlung imaging is feasible, despite scattering and other image degrading artifacts, and it was stated that this imaging could help to appropriately and timely manage patient wellbeing if extrahepatic tracer deposition occurs. The study showed that detection of extrahepatic activity with the post-treatment scan predicted GI ulcers with a sensitivity of $87 \%$ and a specificity of $100 \%$. These predictions help form decisions for an appropriate and timely management strategy when extrahepatic deposition occurs. Minarik et al. (14) also suggested that with proper and accurate dosimetry based on quantitative bremsstrahlung imaging, it is possible to find the relationship between the real uptake relative to the estimate based on pre-treatment tracer to be able to modify a therapy for future treatment and to assess the treatment efficiency.

\subsubsection{Positron emission tomography (PET)}

PET imaging is used with positron-emitting radionuclides. PET detectors detect the 'backto-back' two annihilation photons that are produced when a positron interacts with an ordinary electron $(44,45)$. The photons have identical energy $(511 \mathrm{keV})$ and are emitted simultaneously, in 180-degrees opposing directions. The volume from which these pairs of annihilation photons were emitted can be defined by a technique called annihilation coincidence detection. Because the point of annihilation is very close to the point of positron emission, this gives a good indication of where the radioactive atom was in the body. As a result, PET scanners don't have mechanical collimation to localize radioactive decay as in SPECT, and hence no limit to the field of view. PET scanners instead use electronic collimation that depends on the line of response that passes through the point of annihilation joining the detected photons (Fig. $11 \&$ 12). PET scanners are designed and optimized for imaging all positron-emitting radionuclides at this single energy range $(511 \mathrm{keV})$. By using computed tomography techniques to measure the total radioactivity along the lines that pass at many different angles through the object, two-dimensional images that reflect the concentration of the positron emitting radionuclide in tissues can be reconstructed. The energy of annihilation photons fall in the gamma ray electromagnetic spectrum and the properties of annihilation photons 
are identical to a $511 \mathrm{keV}$ gamma rays, as a result the terms photons and gamma-rays are often used interchangeably when referring to the annihilation photons (46). However, the origins of the two photons are different since annihilation photons do not come from the nucleus. Time of flight (TOF) PET is a technique used to determine the location where the annihilation originated along the line of response (LOR). The time difference between the arrivals of the two annihilation photons at the opposite detectors is measured in TOF PET where annihilation events are localized more accurately in along the line of response (Fig. 11). If the difference in arrival times of photons is $\Delta t$, the location of the annihilation event with respect to the midpoint between detectors is given by Eqn. 13.
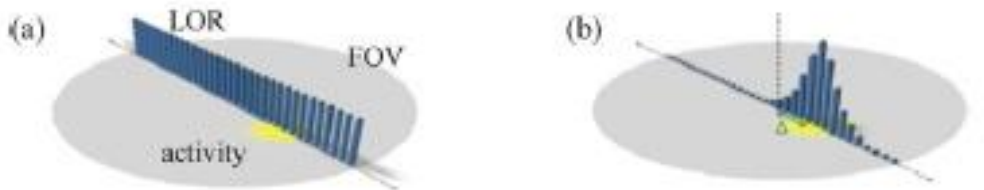

Figure 11: Equal probability assigned to all the pixels along LOR in non-TOF PET (a) and localized counts along the LOR with TOF PET (b) (47).

$$
\Delta d=\frac{\Delta t \times c}{2}
$$



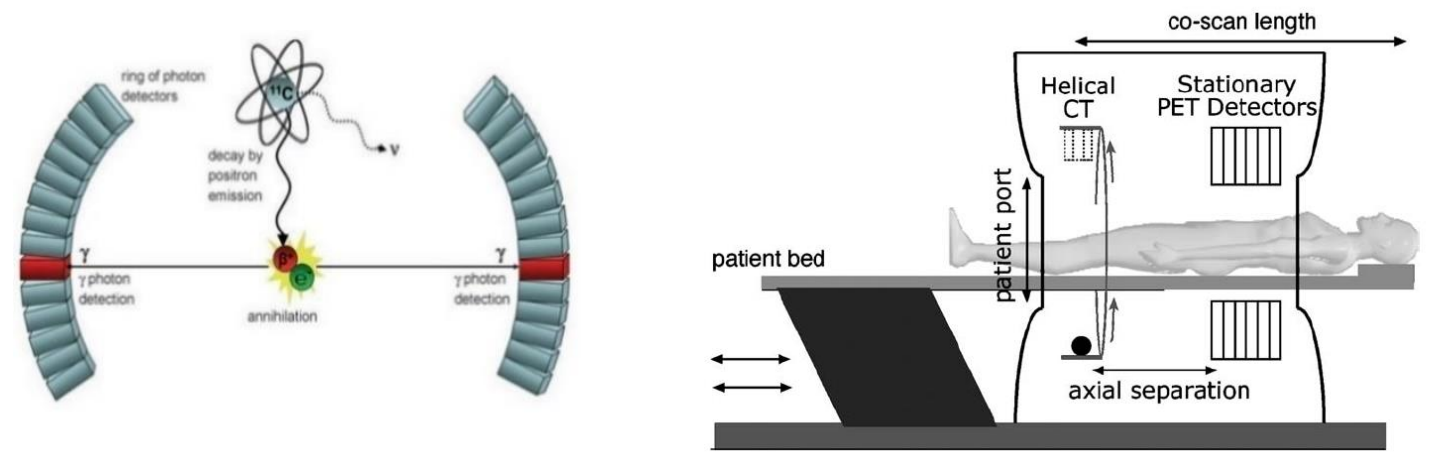

Figure 12: The process of positron-electron annihilation and detection of the annihilation photons (left) and illustration of PET/CT scanner components (48) (right).

Image degrading factors in PET

The main image degrading factors in PET include attenuation, random coincidences and scatter coincidences. As is the case with SPECT, photoelectric effect and Compton scattering are the two major mechanisms by which $511 \mathrm{keV}$ photons interact with matter and which give rise to photon attenuation in PET. Attenuation correction in PET is much more accurate compared to SPECT since the correction is independent of the location of the source in the body. With the inclusion of CT with PET, attenuation correction is achieved with the transmission information from CT although it requires a scaling before it can used since the CT scan is acquired at lower photon energy compared to the $511 \mathrm{keV}$ photons in PET. The CT scan will also provide anatomical localization of PET images giving a better tumor localization compared to PET alone. Random coincidence events in PET occur when two photons originating from different positron decays hit the detector ring within a narrow timing window. Scattered coincidence occurs when one of the photons from an annihilation event undergoes Compton scattering, which can usually be corrected by using energy discrimination.

Tumors are known for their greater metabolic activity accompanied by a greater glucose uptake relative to the surrounding normal tissue (49). Several tracers for glucose metabolism have 
been investigated in PET imaging. Fluorine-18-fluorodeoxyglucose positron emission tomography $\left({ }^{18} \mathrm{~F}-\mathrm{FDG}-\mathrm{PET}\right)$ is the second most used modality in liver cancer imaging because of its significance in the evaluation of extrahepatic disease (50). ${ }^{18} \mathrm{~F}-\mathrm{FDG}$ is a glucose analogue with a biological half-life of 110 minutes, thus accumulation of FDG in tissue is proportional to glucose utilization (51). Greater glucose uptake can be identified with FDG PET which allows for the identification of a tumor foci. However, any focal area of hypermetabolism can give false-positive results (52). PET has the ability to detect liver metastases comparable to MRI and is useful for detecting extrahepatic metastatic disease (53). The limitation of FDG PET is in detecting tumors smaller than $1 \mathrm{~cm}(49,54)$. However, the role of FDG PET/CT in the evaluation and characterization of liver metastases is uncertain due to the heterogeneous uptake of FDG which makes it difficult to exclude the presence of small metastases $(49,50)$.

In 2004, a study showed that ${ }^{90} \mathrm{Y}$ generates pair production at $511 \mathrm{keV}$ that can be detected with a PET camera (55). Various studies have since then demonstrated the feasibility of ${ }^{90} \mathrm{Y}$ microsphere PET/CT imaging as an alternative to ${ }^{90} \mathrm{Y}$ bremsstrahlung SPECT/CT imaging $(17,18)$. ${ }^{90} \mathrm{Y}$ emits 32 positrons per second per MBq (32 per 1,000,000 decays) with a maximum energy of $758 \mathrm{keV}(56)$. Though the rate of pair production is very small, there is a detectable peak of 511 $\mathrm{keV}$ photons surpassing the continuous spectrum of bremsstrahlung photons (Fig 13). Despite the very low positron abundance, various studies have shown ${ }^{90} \mathrm{Y}$ PET/CT images excelling in contrast and resolution compared to bremsstrahlung SPECT/CT $(12,17,18)$. Elschot et al. (17) compared ${ }^{90} \mathrm{Y}$ PET/CT and SPECT/CT in a phantom study with simulated activities. The PET/CT was performed with TOF capability and the image reconstruction included the PSF of the camera while the SPECT/CT was performed using a HEGP collimator with energy window centered at $150 \pm$ $30 \%$ and images were reconstructed with OSEM including the PSF of the detector. The study found that PET estimates activities more accurately than SPECT, in addition, higher contrast recovery coefficient was found on the PET images. The pitfall of the study is that it was a simulated study 
that doesn't represent an actual clinical scenario with acquisition time of $60 \mathrm{~min}$ PET and $120 \mathrm{~min}$ for the SPECT, which gives the PET camera the advantage of collecting more annihilation events. A recent study by Yue et al. (18) investigated patient post-treatment ${ }^{90} \mathrm{Y}$ PET/CT and bremsstrahlung SPECT/CT where the two scans were performed on the same day. PET/CT was done for 30 min per one bed position and the SPECT/CT was done on HEGP collimator with acquisition energy between 100-300 keV. Even though the PET/CT was performed without TOF and coincidence correction capability, the authors concluded that the total activity estimate in the liver is comparable between the two modalities, but PET/CT overestimated ${ }^{90} \mathrm{Y}$ activity in regions with low or no activity. This study lacks correction for prompt coincidence between annihilation and bremsstrahlung photon ${ }^{90} \mathrm{Y}$ imaging. Also, with non TOF capability of PET, the overestimation of photons is inevitable. Thus the total estimate inside the liver could give a satisfactory result, but dosimetry estimation inside tumor and healthy liver region will be compromised as the photons are not spatially corrected, resulting in loss of accuracy.

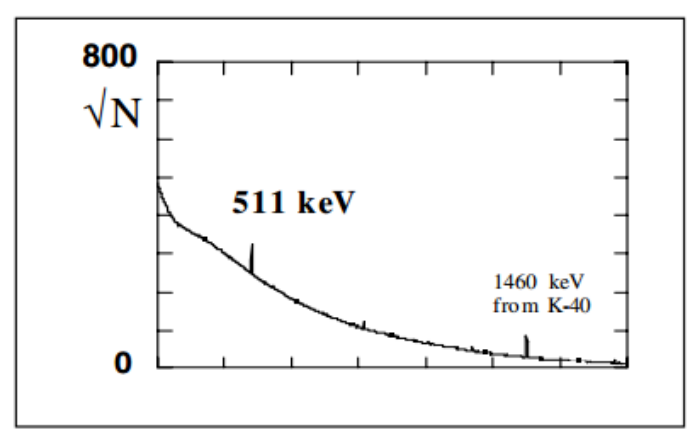

Figure 13: Positron fraction over the continuous bremsstrahlung spectrum as measured by germanium spectrometer (55).

Chapter 3: Framework for Experimental Setup, Data Acquisition and Data Collection

\subsection{Introduction}

A ${ }^{90} \mathrm{Y}$ imaging study, although technically possible, given the inherit characteristics of bremsstrahlung photons current use of the post -RMT bremsstrahlung SPECT/CT is limited to the 
qualitative assessment of the final location of the microspheres (33). Though various authors have come to agreement with the optimal choice of collimator type and acquisition energy window, these choices are not clinically employed as ${ }^{90} \mathrm{Y}$ imaging protocol in many centers. Currently most clinical practices follow the American Association of Physicists in Medicine (AAPM) recommendation for post-treatment ${ }^{90} \mathrm{Y}$ bremsstrahlung SPECT/CT imaging, which recommends the use of medium-energy collimation and energy window centered at $80 \pm 15 \% \mathrm{keV}(68-92 \mathrm{keV})$ (33).

The choice and inclusion criteria for conducting patient research of ${ }^{90} \mathrm{Y}$ bremsstrahlung SPECT/CT imaging varies from one author to the other (Table 2). Patient studies who had whole liver or single lobe treatment might present similar results due to related nomenclatures which requires a cautious interpretation of the presented results. As to whether the whole liver or a single lobe is treated, consistency in the imaging protocol is exceedingly important.

${ }^{90} \mathrm{Y}$ phantom studies are usually done with activity much lower than the actual patient administered activities with great variability across studies, different sphere-to-background ratio, different background concentration and different acquisition time (Table 3). But most studies try to replicate the sphere to background ratios comparable to tumor to healthy liver activity ratios encountered in clinical studies (Table $3 \& 4$ ). The fact that the activities inside the spheres and phantoms are much lower could result in a reduced statistical count in the reconstructed images compromising the accuracy of measured values. 
Table 2: Summary of patient ${ }^{90} \mathrm{Y}$ bremsstrahlung SPECT/CT studies.

\begin{tabular}{lccccc}
\hline Author & $\begin{array}{c}\text { No of } \\
\text { patients }\end{array}$ & $\begin{array}{c}\text { Whole vs. single } \\
\text { lobular } \\
\text { treatment }\end{array}$ & $\begin{array}{c}\text { Total liver } \\
\text { size }(\mathrm{ml})\end{array}$ & $\begin{array}{c}\text { Tumor sizes } \\
\text { (treated } \\
\text { volume) }(\mathrm{ml})\end{array}$ & $\begin{array}{c}\text { Tumor to liver } \\
\text { ratio based on } \\
\text { 99mTc-MAA } \\
\text { SPECT/CT }\end{array}$ \\
\hline $\begin{array}{l}\text { Gulec et al. } \\
(31)\end{array}$ & 40 & Whole & $\begin{array}{c}898.7- \\
3982.0\end{array}$ & $15-984.2$ & $2.8-15.4$ \\
$\begin{array}{l}\text { Garin et al. } \\
(9)\end{array}$ & 36 & Single & NA & 187 (mean) & 7.2 \\
\hline $\begin{array}{l}\text { Elschot et } \\
\text { al. }(17)\end{array}$ & 5 & Whole (4) & $1230-3050$ & $20-383$ & NA \\
\hline $\begin{array}{l}\text { Ilhan et al. } \\
(10)\end{array}$ & 502 & $\begin{array}{c}\text { Whole }(201) \text { and } \\
\text { single }(301)\end{array}$ & NA & NA & $1.65-2.61$ \\
\hline $\begin{array}{l}\text { Yue et al. } \\
(18)\end{array}$ & 15 & Single & NA & $397-2262$ & NA \\
\hline $\begin{array}{l}\text { Siman et } \\
\text { al. }(19)\end{array}$ & 30 & NA & NA & NA & NA \\
\hline
\end{tabular}

Table 3: Summary of ${ }^{90} \mathrm{Y}$ bremsstrahlung SPECT/CT phantom study setups

\begin{tabular}{|c|c|c|c|c|c|c|c|c|}
\hline $\begin{array}{l}\text { Autho } \\
\mathrm{r}\end{array}$ & $\begin{array}{l}\text { Phanto } \\
\mathrm{m}\end{array}$ & $\begin{array}{l}\# \text { of } \\
\text { spheres }\end{array}$ & $\begin{array}{l}\text { Sphere } \\
\text { diamete } \\
r(\mathrm{~mm})\end{array}$ & $\begin{array}{l}\text { Spher } \\
\text { e to } \\
\text { bkg. } \\
\text { ratio }\end{array}$ & $\begin{array}{l}\text { Exp } \\
\text {. vs. } \\
\text { sim. }\end{array}$ & $\begin{array}{l}\text { Acquis } \\
\text { ition } \\
\text { energy } \\
(\mathrm{keV})\end{array}$ & $\begin{array}{l}\text { Collimat } \\
\text { or }\end{array}$ & $\begin{array}{l}\text { Reconstruct } \\
\text { ion alg. } \\
\text { (iter/sub) \& } \\
\text { compensati } \\
\text { on }\end{array}$ \\
\hline $\begin{array}{l}\text { Minar } \\
\text { ik et } \\
\text { al. } \\
(14)\end{array}$ & $\begin{array}{l}\text { Elliptica } \\
1\end{array}$ & 1 & 60 & $\begin{array}{l}\text { No } \\
\text { backg } \\
\text { round } \\
\text { activit } \\
\text { y }\end{array}$ & $\begin{array}{l}\text { Exp } \\
. \& \\
\text { sim. }\end{array}$ & $\begin{array}{l}105- \\
195\end{array}$ & HEGP & $\begin{array}{l}\text { OSEM } \\
(4 / 12) \\
\text { scatter \& } \\
\text { attn. }\end{array}$ \\
\hline $\begin{array}{l}\text { Rong } \\
\text { et al. } \\
(15)\end{array}$ & $\begin{array}{l}\text { Elliptica } \\
1\end{array}$ & 3 & $\begin{array}{l}15,33, \\
55\end{array}$ & $\begin{array}{l}10: 1^{\mathrm{a},} \\
20: 1^{\mathrm{b}}\end{array}$ & $\begin{array}{l}\text { Exp } \\
. \& \\
\text { sim. }\end{array}$ & $\begin{array}{l}100- \\
500^{\mathrm{c}} \\
0- \\
2000^{\mathrm{d}}\end{array}$ & HEGP & $\begin{array}{l}\text { OSEM } \\
(400 / 16) \\
\text { scatter \& } \\
\text { attn. }\end{array}$ \\
\hline $\begin{array}{l}\text { Elsch } \\
\text { ot et } \\
\text { al. } \\
\text { (17) }\end{array}$ & IEC & 6 & $\begin{array}{l}10,13, \\
17,22, \\
28,37\end{array}$ & $9: 1$ & $\begin{array}{l}\text { Exp } \\
\text {. but } \\
\text { sim. } \\
\text { cou } \\
\text { nts }\end{array}$ & $\begin{array}{l}105- \\
195\end{array}$ & HEGP & $\begin{array}{l}\text { OSEM } \\
\text { (8/8) with } \\
\text { PSF \& attn. }\end{array}$ \\
\hline $\begin{array}{l}\text { Siman } \\
\text { et al. } \\
(19)\end{array}$ & IEC & 1 & 37 & 7.8:1 & Exp & $\begin{array}{l}90- \\
125^{\mathrm{c}} \\
310- \\
410^{\mathrm{e}}\end{array}$ & MEGP & $\begin{array}{l}\text { OSEM } \\
(128 / 16) \\
\text { bkg \& attn. }\end{array}$ \\
\hline
\end{tabular}


Bkg: background; Exp: Experimental; sim: simulated; iter/sub: iteration/subset; alg.: algorithm; attn: attenuation

${ }^{a}$ sphere to background ratio in the large and medium sphere.

${ }^{\mathrm{b}}$ sphere to background ratio in the small sphere.

${ }^{\mathrm{c}}$ multiple energy windows were used and reported the values in the table are used for the phantom study

Table 4: Summary of PET/CT studies

\begin{tabular}{|c|c|c|c|c|c|c|c|}
\hline Author & $\begin{array}{c}\text { Phantom } \\
\text { type }\end{array}$ & $\begin{array}{c}\text { No of } \\
\text { patients }\end{array}$ & $\begin{array}{c}\text { Sphere } \\
\text { diameter( } \\
\text { mm) }\end{array}$ & $\begin{array}{l}\text { Sphere to } \\
\text { bkg. ratio }\end{array}$ & $\begin{array}{c}\text { Exp. vs. } \\
\text { sim. }\end{array}$ & TOF & $\begin{array}{c}\text { Scatter } \\
\text { and attn. } \\
\text { correction }\end{array}$ \\
\hline $\begin{array}{l}\text { Elschot et } \\
\text { al. (17) }\end{array}$ & IEC & 6 & $\begin{array}{l}10,13, \\
17,22, \\
28,37\end{array}$ & $9: 1$ & $\begin{array}{l}\text { Exp, but } \\
\text { sim. } \\
\text { counts }\end{array}$ & TOF & Corrected \\
\hline $\begin{array}{l}\text { Yue et al. } \\
\text { (18) }\end{array}$ & NA & 15 & NA & NA & $\begin{array}{c}\text { Patient } \\
\text { data }\end{array}$ & $\begin{array}{l}\text { Non- } \\
\text { TOF }\end{array}$ & Corrected \\
\hline $\begin{array}{l}\text { Willowson } \\
\text { et al. (57) } \\
\text { Attarwala } \\
\text { et al. (58) }\end{array}$ & IEC & NA & $\begin{array}{l}10,13, \\
17,22, \\
28,37\end{array}$ & $8: 1$ & Exp. & TOF & Corrected \\
\hline $\begin{array}{l}\text { Martí- } \\
\text { Climent et } \\
\text { al. }(56)\end{array}$ & Jaszczak & 10 & $\begin{array}{c}31.3, \\
28.1, \\
21.8, \\
16.113 .3\end{array}$ & 2.5 & Exp. & $\begin{array}{l}\text { TOF, } \\
\text { Non- } \\
\text { TOF }\end{array}$ & Corrected \\
\hline $\begin{array}{l}\text { Willowson } \\
\text { et al. (59) }\end{array}$ & IEC & NA & $\begin{array}{l}10,13 \\
17,22 \\
28,37\end{array}$ & $8: 1$ & $\begin{array}{l}\text { Exp. but } \\
\text { sim. } \\
\text { counts } \\
\text { (7 days) }\end{array}$ & $\begin{array}{l}\text { TOF, } \\
\text { Non- } \\
\text { TOF }\end{array}$ & Corrected \\
\hline $\begin{array}{l}\text { Rowley et } \\
\text { al. (60) }\end{array}$ & IEC & 10 & $\begin{array}{l}10,13, \\
17,22, \\
28,37\end{array}$ & & $\begin{array}{l}\text { Exp. but } \\
\text { sim. } \\
\text { counts } \\
(12 \mathrm{hr})\end{array}$ & TOF & Corrected \\
\hline
\end{tabular}

3.2 Materials and Methods

\subsubsection{Patient study:}

Patient data were acquired from Baptist Hospital of Miami as part of a retrospective study approved by the Florida International University Institutional Review Board. Therefore, written 
patient informed consent was not sought nor documented and image data were handled anonymously.

\section{${ }^{99 m}$ TC-MAA SPECT/CT imaging}

All patients had undergone ${ }^{99 \mathrm{~m} T c-M A A}$ SPECT/CT scan before RMT, of which twelve of those patients were used for comparison of tumor to liver ratios for our study as the ${ }^{99 \mathrm{~m}} \mathrm{Tc}-\mathrm{MAA}$ SPECT/CT data from the remaining seven patients had been transferred to a different location at the time of the study and so unavailable. Mean administered ${ }^{99 \mathrm{~m} T c-M A A}$ was $190 \mathrm{MBq}$. SPECT/CT data were collected for patients whose LSF $\leq 5 \%$ to minimize error due to extrahepatic deposition consistent with another group (19). The acquisition energy window was centered at $140 \mathrm{keV}$ for ${ }^{99 m}$ Tc-MAA SPECT with $128 \times 128$ projection matrix over $360^{\circ}$ for 20 seconds per azimuth and with a low-energy high-resolution collimator. Image reconstruction was done using the ordered subset expectation maximization (OSEM) algorithm with 3 iterations and 16 subsets, $132 \times 132$ matrix and $4.664 \times 4.664 \mathrm{~mm}^{2}$ pixel size.

\section{${ }^{90} Y$ microsphere SPECT/CT imaging}

In total, SPECT/CT data of 19 patients who underwent RMT with ${ }^{90}$ Y-labeled resin microspheres (SIR-spheres; SIRTEX) were acquired. ${ }^{90} \mathrm{Y}$ bremsstrahlung SPECT/CT was performed with a medium energy general purpose collimator energy window centered on $90 \mathrm{keV}$ $\pm 15 \%, 35$ seconds per azimuth for $2 \times 64$ views over $360^{\circ}$ (currently a standard protocol at our center). Image reconstruction for ${ }^{90} \mathrm{Y}$ SPECT was performed using Astonish from Philips Medical Systems. Astonish is a 3D OSEM algorithm with built-in noise reduction that incorporates attenuation and scatter corrections using the CT attenuation map and the effective source scatter estimation method respectively, and also incorporates depth-dependent resolution recovery $(61,62)$. The ${ }^{90} \mathrm{Y}$ bremsstrahlung SPECT images were reconstructed with 4 iterations and 8 subsets, $132 \times$ 132 matrix and $4.664 \times 4.664 \times 4.664 \mathrm{~mm}^{3}$ voxel size. 


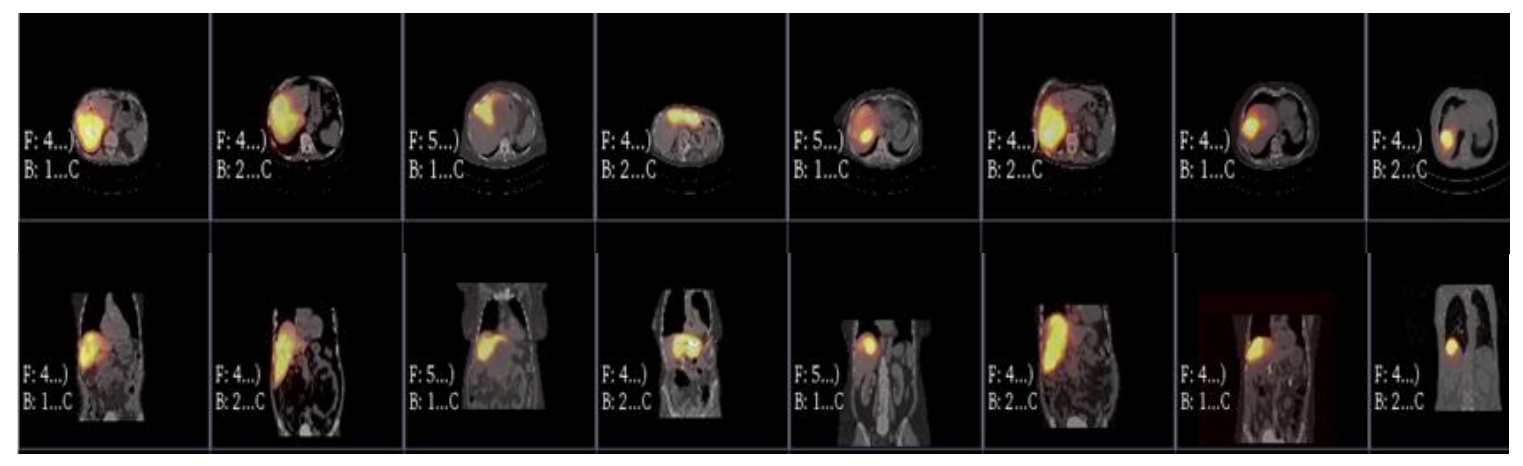

Figure 14: Patient ${ }^{90} \mathrm{Y}$ microsphere SPECT/CT images in axial (upper) and coronal (lower) views. 
Table 5: Patient characteristics.

\begin{tabular}{|c|c|c|}
\hline Patient & $\begin{array}{l}{ }^{90} \mathrm{Y} \text { administered } \\
\text { activity }(\mathrm{MBq})\end{array}$ & $\begin{array}{c}\text { 99m Tc-MAA SPECT/CT } \\
\text { available }(\mathrm{Y} / \mathrm{N})\end{array}$ \\
\hline 1 & 547.60 & $\mathrm{~N}$ \\
\hline 2 & 555.00 & $\mathrm{Y}$ \\
\hline 3 & 558.70 & $\mathrm{~N}$ \\
\hline 4 & 569.80 & $\mathrm{Y}$ \\
\hline 5 & 758.50 & $\mathrm{Y}$ \\
\hline 6 & 780.70 & $\mathrm{Y}$ \\
\hline 7 & 888.00 & $\mathrm{~N}$ \\
\hline 8 & 965.70 & $\mathrm{Y}$ \\
\hline 9 & 999.00 & Y \\
\hline 10 & 1061.90 & $\mathrm{~N}$ \\
\hline 11 & 1202.50 & $\mathrm{Y}$ \\
\hline 12 & 1195.10 & $\mathrm{~N}$ \\
\hline 13 & 1235.80 & $\mathrm{Y}$ \\
\hline 14 & 1261.70 & $\mathrm{Y}$ \\
\hline 15 & 1435.60 & $\mathrm{Y}$ \\
\hline 16 & 1517.00 & $\mathrm{Y}$ \\
\hline 17 & 2072.00 & $\mathrm{Y}$ \\
\hline
\end{tabular}

\subsubsection{Phantom study:}

The Jasczak (Fig. 15) phantom (6 liter) which simulates a human torso was used. Eight fillable spherical inserts of inner diameter 2, 8, 10, 12, 16, 25, 31 and $34 \mathrm{~mm}$ were inserted inside the phantom to mimic variable size tumors inside the human liver (Fig. 14). For experiment 1, the 
spheres and background were filled with Yttrium-90 (III) chloride solution (PerkinElmer Inc., USA) of total activity 27 and $255 \mathrm{MBq}$ respectively with an approximate sphere to background activity concentration ratio of $13: 1$. For the second experiment, ${ }^{90} \mathrm{Y}$ was extracted from SIRSpheres using a saline solution. The solution was set for about $30-45$ minutes for ${ }^{90} \mathrm{Y}$ to separate from the microspheres the microspheres to settle to the bottom. The ${ }^{90} \mathrm{Y}$ solution was then extracted with a needle syringe to minimize disruption of the mixture. In both experiments ${ }^{90} \mathrm{Y}$ activity was diluted and measured in a $60 \mathrm{ml}$ vial before adding to the spheres and the activity inside each sphere was also measured afterwards using a Capintec dose calibrator (read out scale factor $=10$ ). The increased activity in the spheres for experiment 2 was to be able to collect enough annihilation events for PET/CT imaging (Table 6). The reported values in Table 6 are based on dilution equation. Although the reading from the dose calibrator is recorded, the calibrator has \pm 10 read out error for mCi measurement which might not give accurate measurement for activities in $\mu \mathrm{Ci}$. For experiment 2 , the expected values from the dilution equation might not represent exact values in the sphere as there could be microspheres associated with ${ }^{90} \mathrm{Y}$ invisible to the naked eye.
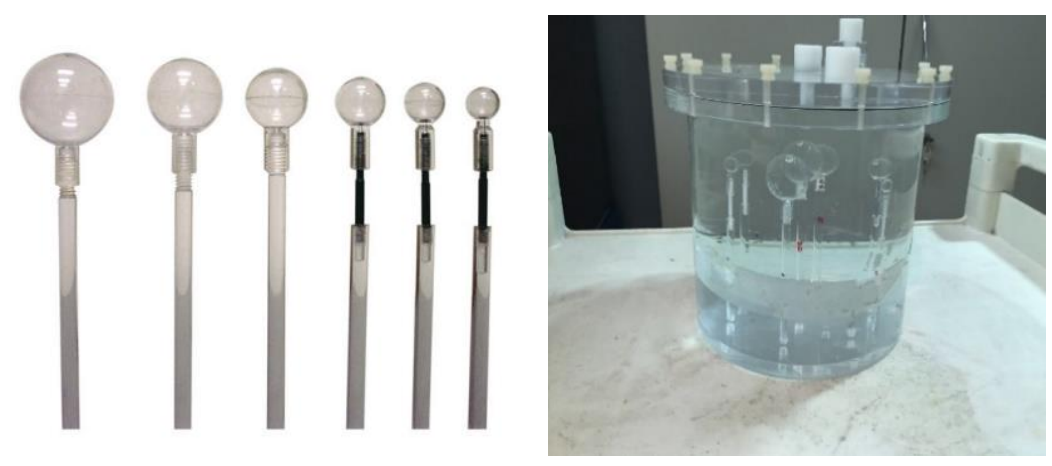

Figure 15: Different size hollow spheres (left) and Jasczak phantom with spherical inserts (right). 
Table 6: Phantom activity

$$
\text { Activity (MBq) }
$$

\begin{tabular}{|c|c|c|c|}
\hline Sphere no & $\begin{array}{l}\text { Sphere size in } \\
\text { diameter }(\mathrm{mm})\end{array}$ & Experiment 1 & Experiment 2 \\
\hline $\mathrm{S} 1$ & 34 & 11.10 & 33.30 \\
\hline $\mathrm{S} 2$ & 31 & 8.88 & 12.65 \\
\hline S3 & 25 & 4.44 & 7.15 \\
\hline S4 & 16 & 1.11 & 4.87 \\
\hline S6 & 12 & 0.56 & 1.67 \\
\hline S7 & 10 & 0.28 & 0.85 \\
\hline S8 & 8 & 0.14 & 0.40 \\
\hline S9 & 2 & $\sim 0$ & NA \\
\hline
\end{tabular}

Image acquisition and reconstruction

\section{SPECT/CT imaging}

Data were acquired on a dual-head Philips Precedence 16P SPECT/CT (Philips Medical Systems Inc., USA) at the Baptist Hospital of Miami. Two separate experiments were performed for SPEC/CT imaging. The first experiment involves imaging with two different collimators, MEGP and HEGP. The SPECT/CT imaging setup with the MEGP collimator was identical to the patient studies in terms of imaging window, collimator and image reconstruction. Details of the SPECT/CT experiments are presented in Table 7. 
Table 7: SPEC/CT imaging parameters

\begin{tabular}{|c|c|c|c|}
\hline & \multicolumn{2}{|l|}{ Experiment 1} & Experiment 2 \\
\hline & Imaging 1 & Imaging 2 & Imaging 1 \\
\hline Camera brand & Philips Precedence 16 & Philips Precedence 16 & Philips Precedence 16 \\
\hline Dual-head & Yes & Yes & Yes \\
\hline Field of view (FOV) & $60 \mathrm{~cm}$ & $60 \mathrm{~cm}$ & $60 \mathrm{~cm}$ \\
\hline Crystal type & $\mathrm{NaI}(\mathrm{Tl})$ & $\mathrm{NaI}(\mathrm{Tl})$ & $\mathrm{NaI}(\mathrm{Tl})$ \\
\hline Collimator type & MEGP & HEGP & MEGP \\
\hline Energy window & $90 \pm 15 \% \mathrm{keV}$ & $105-195(150 \pm 30 \%)$ & $90 \pm 15 \% \mathrm{keV}$ \\
\hline $\begin{array}{l}\text { Reconstruction } \\
\text { algorithm }\end{array}$ & Astonish OSEM & Astonish OSEM & Astonish OSEM \\
\hline Iterations/subsets & $4 / 8$ & $8 / 8$ & $4 / 8$ \\
\hline $\begin{array}{l}\text { Post reconstruction } \\
\text { filter }\end{array}$ & NONE & NONE & NONE \\
\hline Acquisition time & $18 \mathrm{~min}$ & $18 \mathrm{~min}$ & $18 \mathrm{~min}$ \\
\hline Matrix size $\mathrm{CT}$ & $512 \times 512$ & $512 \times 512$ & $512 \times 512$ \\
\hline Matrix size & $132 \times 132$ & $132 \times 132$ & $132 \times 132$ \\
\hline Voxel size & $\begin{array}{l}4.664 \times 4.664 \times 4.664 \\
\mathrm{~mm}^{3}\end{array}$ & $\begin{array}{l}4.664 \times 4.664 \times 4.664 \\
\mathrm{~mm}^{3}\end{array}$ & $\begin{array}{l}4.664 \times 4.664 \times 4.664 \\
\mathrm{~mm}^{3}\end{array}$ \\
\hline
\end{tabular}

\section{PET/CT imaging}

PET/CT data were acquired on a GE Discovery 690 TOF scanner equipped with FOV of $70 \mathrm{~cm}$. PET data were acquired in the identical imaging protocol as the patient ${ }^{18} \mathrm{~F}-\mathrm{FDG}$ PET/CT imaging. The energy window was centered at $511 \mathrm{keV}$. Two experiments were performed; 15 or 30 min scan times. Single bed position was chosen due to the small size of the phantom where major counts are detected along the center rings compared to a standard human size where significant detection also occurs along the edge ring detectors. The image reconstruction algorithm included correction for random coincidences, scatter and attenuation. Reconstructed image matrix 
was $128 \times 128$ with $5.47 \times 5.47 \times 5.47 \mathrm{~mm}^{3}$ voxel size. Table 8 presents details of the imaging parameter for the PET/CT imaging.

Table 8: PET/CT imaging parameters

\begin{tabular}{lll}
\hline & Experiment 1 & \multicolumn{1}{c}{ Experiment 2} \\
\hline Camera brand & GE Discovery 690 & GE Discovery 690 \\
\hline Crystal type & Germanium & Germanium \\
\hline Energy window & $511 \mathrm{keV}$ centered & $511 \mathrm{keV}$ centered \\
\hline FOV of CT & $70 \mathrm{~cm}$ & $70 \mathrm{~cm}$ \\
\hline Current CT & $62 \mathrm{~mA}$ & $62 \mathrm{~mA}$ \\
\hline Distance from sample & $10 \mathrm{~cm}$ & $10 \mathrm{~cm}$ \\
\hline Number of projections/views & 83 & 83 \\
\hline Bed position & 1 & 1 \\
\hline Acquisition time & $15 \mathrm{~min}$ & $30 \mathrm{~min}(15 \mathrm{~min}$ per bed $)$ \\
\hline Reconstruction algorithm & Time of Flight & Time of Flight \\
\hline Matrix CT & $512 \times 512$ & $512 \times 512$ \\
\hline Matrix SPECT & $128 \times 128$ & $128 \times 128$ \\
\hline
\end{tabular}

\subsubsection{Image processing}

A MATLAB ${ }^{\circledR}$ algorithm was developed to import and export images for Region and

Volumes of Interests (ROIs and VOIs) generation, semi-automatic tumor segmentation, activity estimation, absorbed dose estimation and statistical and mathematical analysis. For the patient studies, VOIs were drawn manually on the CT images slice by slice for liver segmentation. Binary masks from the CT images were mapped onto the respective SPECT scans. For the phantom study, eight circular VOIs were manually drawn on the CT slices, where knowledge of the phantom composition allowed us to identify the spheres and made sure the volumes in VOIs were consistent with the true measured volumes. Background VOIs consisted of all voxels within the phantom boundary excluding voxels that belong to the sphere VOIs. A single slice through the center of the coplanar spheres was used for generating 8 circular ROIs equal to the spheres' inner diameter. A 
background ROI defined in the same slice consisted of all voxels within the phantom except the sphere ROI.

\subsubsection{Statistical Analysis}

Quantitative parameters are presented as mean \pm SD and ranges. Linear regressions were generated between administered activities (independent variable) and cps for predicting calibration factors. Slope, R-squared, standard error and 95\% confidence interval of the regression models are reported. Pearson correlation coefficients and were used to test for significance of correlations between TLRs from ${ }^{99 m}$ Tc-MAA and ${ }^{90} \mathrm{Y}$ SPECT and between administered activity and absorbed doses and $\mathrm{p}$ values reported. Statistical analyses were deemed significant as having a $\mathrm{p}$ value less than 0.05 . All analyses were performed with Minitab ${ }^{\circledR}$ software package (version 17).

\subsection{Results and Discussion}

Five different reconstructed images from four phantom acquisitions have been used for the analyses. Figure 16 shows the position of the phantom during acquisition and the energy resolutions for the two collimators of experiment 1 . For the HEGP collimator, the energy window is higher and has the ability of stopping the high energy bremsstrahlung photons. As a result, the visibility of the smaller photons has increased (Fig. 17). Although the vendor attenuation and scatter correction is not well optimized for ${ }^{90} \mathrm{Y}$ imaging, based on visual inspection the inclusion of attenuation and scatter correction in the OSEM reconstruction algorithm appears to give a better resolution image compared to using the OSEM algorithm without the correction (Fig. 17).

The PET/CT imaging of the first experiment setup didn't give significant counts for $15 \mathrm{~min}$ imaging, thus wasn't used for analysis. The minimum count rate in PET/CT imaging of the first experiment could be associated with the small concentration inside the spheres $(0.56 \mathrm{MBq} / \mathrm{ml})$ in addition to the shorter imaging time. Tapp et al. (63) has performed sequential ${ }^{90} \mathrm{Y}$ PET/CT imaging for 5 days. The authors found that the minimum detectable concentration for phantom inserts of 10 
min PET/CT imaging was $1 \mathrm{MBq} / \mathrm{ml}$. Elschot et al. (17) used $2.4 \mathrm{MBq} / \mathrm{ml}$ inside spherical inserts with 60 min PET/CT acquisition scan, though the imaging time used by the authors doesn't represent actual patient scanning (10-30 min). For the second experiment, the concentration inside the largest spheres was approximately $1.67 \mathrm{MBq} / \mathrm{ml}$. This change in concentration doesn't seem to have an impact on the SPECT/CT imaging (Fig. 18). However, the change in concentration as well as a prolonged acquisition time gave better result of the PET/CT imaging; 3 out of the 7 spheres are distinguishable (Fig. 19). Marti-Climent et al. (56) claimed a more realistic clinical condition of ${ }^{90} \mathrm{Y}$ PET/CT imaging was performed where the authors used $0.207 \mathrm{MBq} / \mathrm{ml}$ and $0.199 \mathrm{MBq} / \mathrm{ml}$ concentrations inside the spheres and background respectively with acquisition time of $39 \mathrm{~min}$.

There is great variability in ${ }^{90} \mathrm{Y}$ phantom experimental designs in the literature, with different sphere to background concentration ratios, different background concentrations and different acquisition times (56). For a PET/CT study specifically these variations will impact the count statistics as ${ }^{90} \mathrm{Y}$ PET is concentration dependent (18).
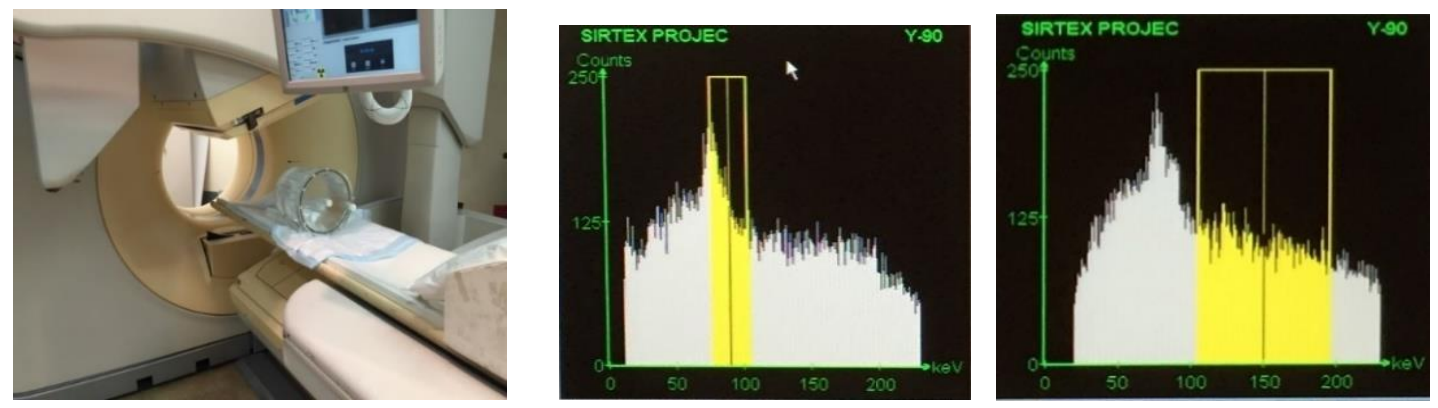

Figure 16: SPECT/CT (left) Phantom imaging, and energy resolution of the two detectors centered for the MEGP (middle) and HEGP (right).
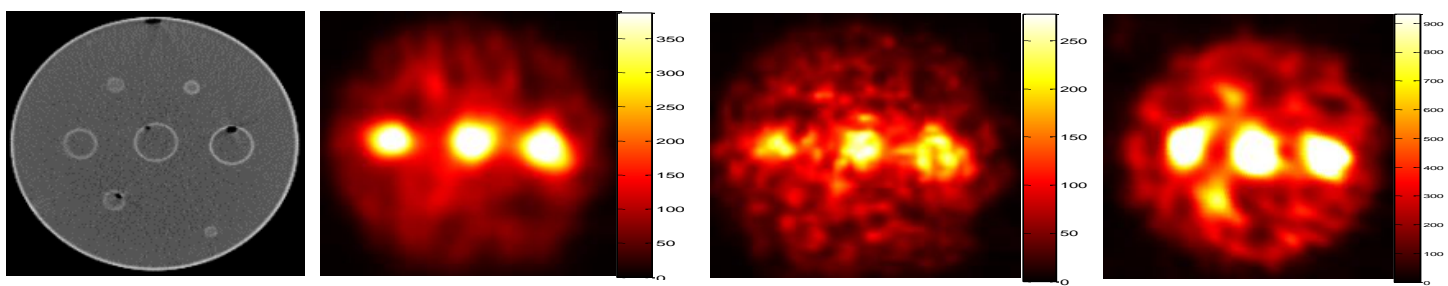

Figure 17: CT (left) and SPECT reconstructed with Astonish OSEM MEGP, OSEM only MEGP and Astonish OSEM HEGP consecutively of experiment 1. 


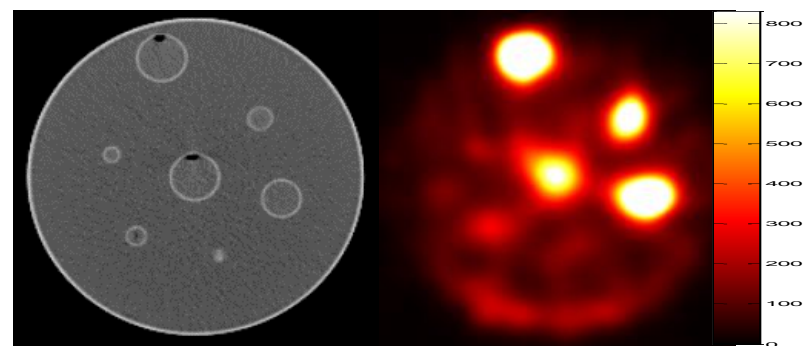

Figure 18: CT (left) and SPECT (right) scans imaged with the MEGP collimator of experiment 2.
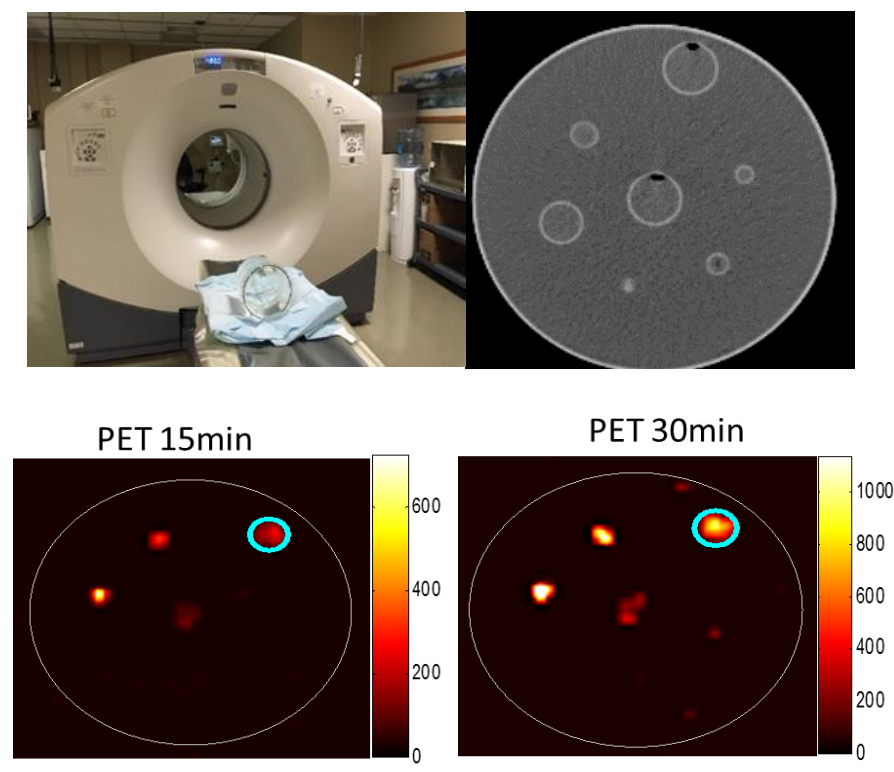

Figure 19: PECT/CT scanner and CT image (upper) and PET 15 \& 30min images (lower).

\subsection{Conclusion}

We have collected ${ }^{99 \mathrm{~m}} \mathrm{Tc}-\mathrm{MAA}$ and ${ }^{90} \mathrm{Y}$ microsphere SPECT/CT images for quantitative comparison and dosimetry purpose. Variable ${ }^{90} \mathrm{Y}$ phantom experiments were performed to investigate the optimal combination of imaging window, reconstruction algorithm, collimator type and scan duration. A large variation was observed in ${ }^{90} \mathrm{Y}$ experiment design among various authors. Although these variations don't impact ${ }^{90} \mathrm{Y}$ bremsstrahlung SPECT/CT study due to the satisfactory count statistics obtained, care must be taken while designing ${ }^{90} \mathrm{Y}$ PECT/CT imaging as there is only $32 \mathrm{ppm}$ of annihilation events, requiring either a larger duration of scan or a high activity concentration compared to SPECT/CT imaging. 


\section{Chapter 4: Spatial Resolution Recovery algorithm}

\subsection{Introduction}

Spatial resolution is the ability of an imaging device to provide a sharp (detailed) image. In nuclear medicine, a number factors contribute to the loss of image sharpness, such as collimator resolution, intrinsic resolution and patient movement. In gamma cameras with absorptive collimators, the major limiting factor of image resolution is the collimator resolution. In PET though, the intrinsic resolution of the detectors, i.e. the size of individual detector elements, limit the image resolution. Collimator resolution depends on the diameters of the holes and the sourceto-detector distance, which contribute to image blurring. Patient movement, such respiration and cardiac motion, can be troublesome when imaging is performed for a longer period, but which can be corrected by using gated-imaging techniques to minimize motion blurring. Spatial resolution in SPECT is characterized using the profile of the reconstructed image of a line source or point source, usually a ${ }^{99 \mathrm{~m}} \mathrm{Tc}$ point source, placed in the field of view (FOV) of the camera. The profile through the center image of the point source gives the point spread function (PSF). Spatial resolution is then characterized by the full width at half maximum (FWHM) of the PSF. The intrinsic spatial resolution of PET is a Gaussian function with FWHM that changes with the location of the source from the two detectors, highest at the face of either detector.

Image restoration or spatial resolution recovery allows for correction of collimator-detector blur which gives an improved spatial resolution. In image restoration the problem is finding an estimate of the input image $f(x, y)$, given the noisy and blurred image $g(x, y)$ and PSF of the detector written as:

$$
g(x, y)=p s f(x, y) \otimes f(x, y)
$$

In ${ }^{90} \mathrm{Y}$ bremsstrahlung SPECT and PET imaging, correction for detector response involves deconvolution filtering, iterative reconstruction methods that involve the PSF model of the detector 
with or without the inclusion of post reconstruction filters such as Gaussian or Wiener filter and MC simulation to generate the collimator-detector response kernels which are associated in the iterative reconstruction algorithm. The MC method is the best suited method of correction, but is not widely available in the clinic to date. As a result there is a need for a more practical correction for the collimator-detector response in ${ }^{90} \mathrm{Y}$ bremsstrahlung SPECT and PET imaging.

\subsection{Materials and Methods}

It was mentioned earlier that SPECT has limited resolution due to image degrading factors modifying the linear relationship between the projection data and the image data by including the effects of attenuation, scatter and collimator detector response functions. Due to the random nature of radioactive decay in SPECT and PET a Poisson model is appropriate for emission data analysis. The Poisson model provides the probability of acquiring the projection count distribution that was measured, $\mathrm{P}$, given an estimated distribution of activity in the emission object, $f$, which can be represented by the product of probabilities for individual pixels as follows:

$$
\mathrm{g}=\mathbf{P} f \Leftrightarrow p(s, \theta)=\iint f(x, y) a(x, y) s(x, y) d(x \cos \theta+y \operatorname{sinc} \theta-s) d x d y \quad 15
$$

The Poisson model is well approximated by a Gaussian function provided measured counts are reasonably high, giving a more simplified form of Eqn. 16 as,

$$
G \sim \text { Poisson }\{P f+\varepsilon\}
$$

With a Poisson distribution of $\lambda$ and $\varepsilon$ representing the additive noises such as scattered counts. In image restoration, the real image estimate is sought given the $\mathrm{g}$ as a reconstructed form of vector G.

\subsubsection{The Richardson - Lucy deconvolution method}

The Richardson-Lucy (RL) technique was used for post-reconstruction image deconvolution. The RL method was initially developed from Bayes's theorem that relates conditional probabilities by taking into account the statistical fluctuations in a signal. The method is an iterative expectation maximum likelihood deconvolution algorithm (Eqn. 17) where images 
degraded by the PSF of the detector, Poisson statistics and additive noises, are corrected $(64,65)$. The choice of the maximum likelihood algorithm has the benefit of producing good quality images in the presence of high noise levels by preserving positive values through accounting for fluctuations in the signal and thus limiting noise amplification (65). This method is especially useful in emission tomography imaging where Poisson modeling is found to be appropriate as mentioned earlier. The Bayes' theorem is stated as,

$$
P(x \mid y)=\frac{P(y \mid x) P(x)}{\int P(y \mid x) P(x) d x}
$$

Where $P(y \mid x)$ is the conditional probability of an event y given $\mathrm{x}, P(x)$ is the probability of an event $x, P(x \mid y)$ is the conditional probability of $x$ given $y$ (inverse probability). Relating the Bayes' theorem to the emission tomography imaging explained in Eqns. $16 \& 17$ in isoplane, the probability $P(x)$ can be related to the radioactive distribution of object $f(x, y, z)$ in $2 \mathrm{D}$, the conditional probability $P(y \mid x)$ can be related to the PSF of the detector for a point source $\operatorname{PSF}(x, y, z$,$) and the$ probability $P(y)$ can be related to the degraded image $g(x, y)$. From the inverse relationship of $f(x, y, z)$ and $g(x, y, z)$, the iterative algorithm can be written as,

$$
f_{i}(x, y, z)=\left\{\left[\frac{g(x, y, z)}{f_{i-1}(x, y, z) \otimes P S F(x, y, z)}\right] \otimes P S F(x, y, z)\right\} f_{i-1}(x, y, z)
$$

The algorithm requires an initial guess of the $f_{0}(x, y, z)$ to start the iteration where $f_{0}(x, y, z)=g(x, y, z)$. In our study the PSF was fixed and the only iterative maximum likelihood estimate was the image.

\subsubsection{Modeling of the point spread function of the detector}

The PSF of the collimator-detector response was modeled by a 3D Gaussian kernel creating a Poisson realization for each reconstructed pixel count. The Gaussian function for a 3D spatial is expressed as,

$$
P S F(x, y, z)=\frac{(2 \pi)^{-\frac{3}{2}}}{\sigma_{s_{x}} \sigma_{s_{y}} \sigma_{s_{z}}} \times \exp \left(-\frac{1}{2}\left[\frac{x^{2}}{\sigma_{s_{x}}^{2}}+\frac{y^{2}}{\sigma_{s_{y}}^{2}}+\frac{z^{2}}{\sigma_{s_{z}}^{2}}\right]\right)
$$


Where $\sigma_{\mathrm{Sx}}, \sigma_{\mathrm{Sy}}, \sigma_{\mathrm{Sz}}$ represent the standard deviation in the $\mathrm{x}, \mathrm{y}$, and $\mathrm{z}$ directions. For a spatially invariant PSF i.e. the response to a point source of activity is the same for all points in the object, Eqn. 19 reduces to,

$$
\operatorname{PSF}(x, y, z)=\frac{1}{(2 \pi)^{\frac{3}{2}} \sigma^{3}} e^{-\left(x^{2}+y^{2}+z^{2}\right) / 2 \sigma^{2}}
$$

Since scanner spatial resolution is defined in terms of FWHM of the Gaussian function of the PSF, the $\sigma$ was found by relating it with FWHM of the detector,

$$
\frac{1}{2}=\exp \left(-\frac{1}{2}\left(\frac{\mathrm{FWHM}}{2 \sigma}\right)^{2}\right) \Leftrightarrow \mathrm{FWHM}=\sqrt{8 \ln (2)} \mathrm{x} \sigma
$$

The iteration number for the algorithm was chosen so that convergence is reached at the point of maximum likelihood where the resolution recovery coefficient for the $34 \mathrm{~mm}$ sphere was at its maximum value. This point also corresponded to the smallest associated root mean square error (RMSE) between two consecutive iterative image estimates (Fig. 20). Improvements in the quantitative quality ${ }^{90} \mathrm{Y}$ SPECT images were evaluated using contrast to noise ratio (CNR) and contrast recovery coefficients $\left(\mathrm{Q}_{\mathrm{H}}\right)(17)$ for the patient and phantom studies respectively given by Eqns. 22 and 23. These quantitative measures were calculated by two methods: regions of interest (ROIs) drawn through the centers of the spheres equal to the inner diameter of the spheres and volumes of interest (VOIs) drawn on CT slices that cover the entire sphere volume with equal diameters to the inner diameter of the spheres. This comparative measurement of quantitative improvement in the SPECT/CT and PET/CT images based on ROIs, as opposed to a VOI method was investigated to give an indication of the most appropriate approach for dosimetry application.

$$
\begin{gathered}
C N R=\frac{M_{T}-M_{B}}{\sqrt{M_{B}}} \\
Q_{H}=\frac{C_{S} / C_{B}-1}{R-1} \times 100 \\
R M S E_{f_{i}}=\left[\sum_{i}^{N} \frac{\left(f_{i}-f_{i-1}\right)^{2}}{N}\right]^{1 / 2}
\end{gathered}
$$


$M_{T}$ is the mean count in tumor VOIs, $M_{B}$ is the mean count in healthy liver VOIs, $C_{S}$ is the mean count in the sphere VOIs, $C_{B}$ is the mean count in the background VOIs and $R$ is the true sphere to background ratio

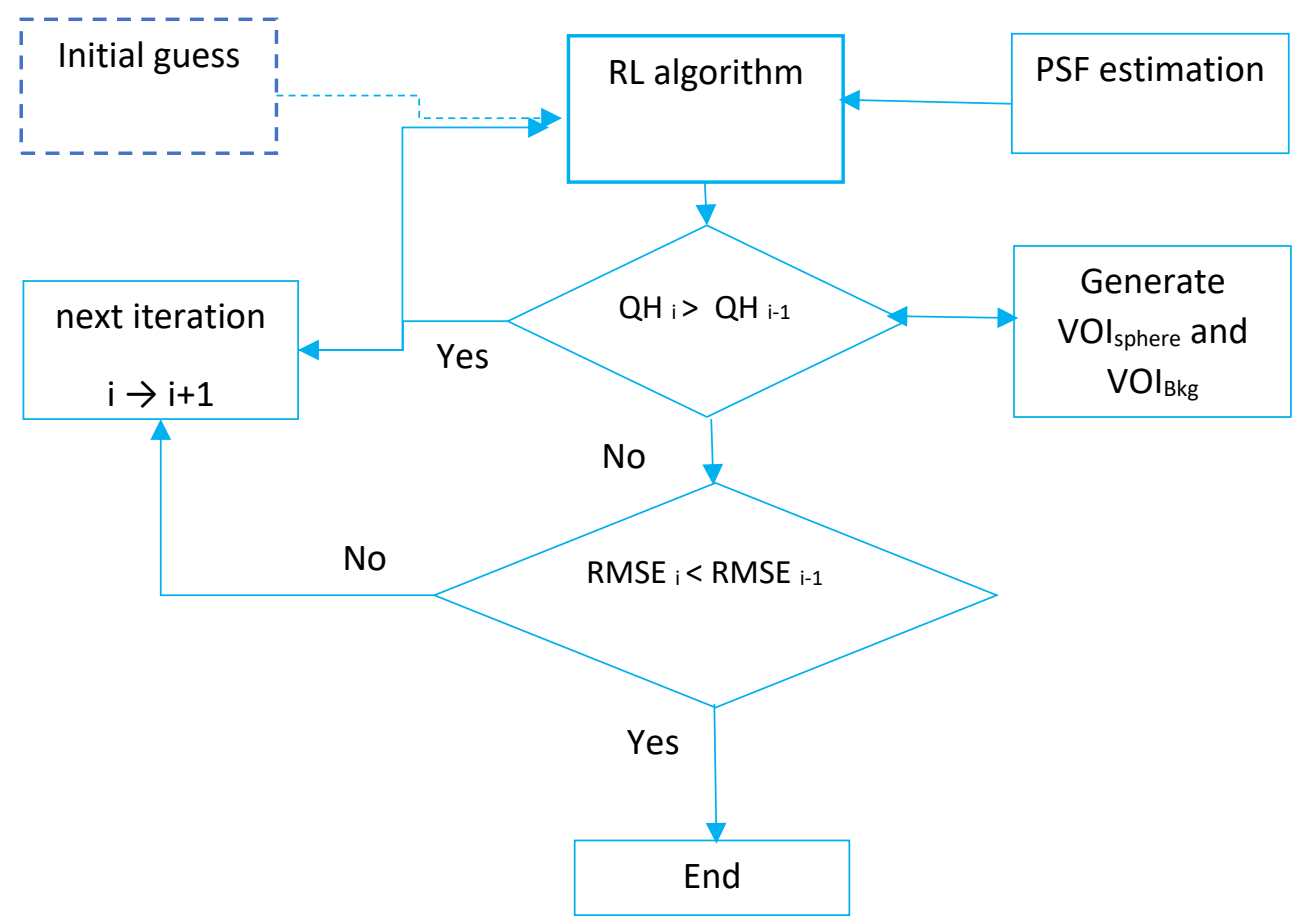

Figure 20: Block diagram of the RL algorithm employed.

\subsection{Results and Discussion}

\subsection{SPET/CT study}

Figure 21 shows the SPECT slice through the hot spheres of the phantom image generated using the MEGP and HEGP collimator corrected for spatial resolution using variable FWHM of the PSF and iterations number. 


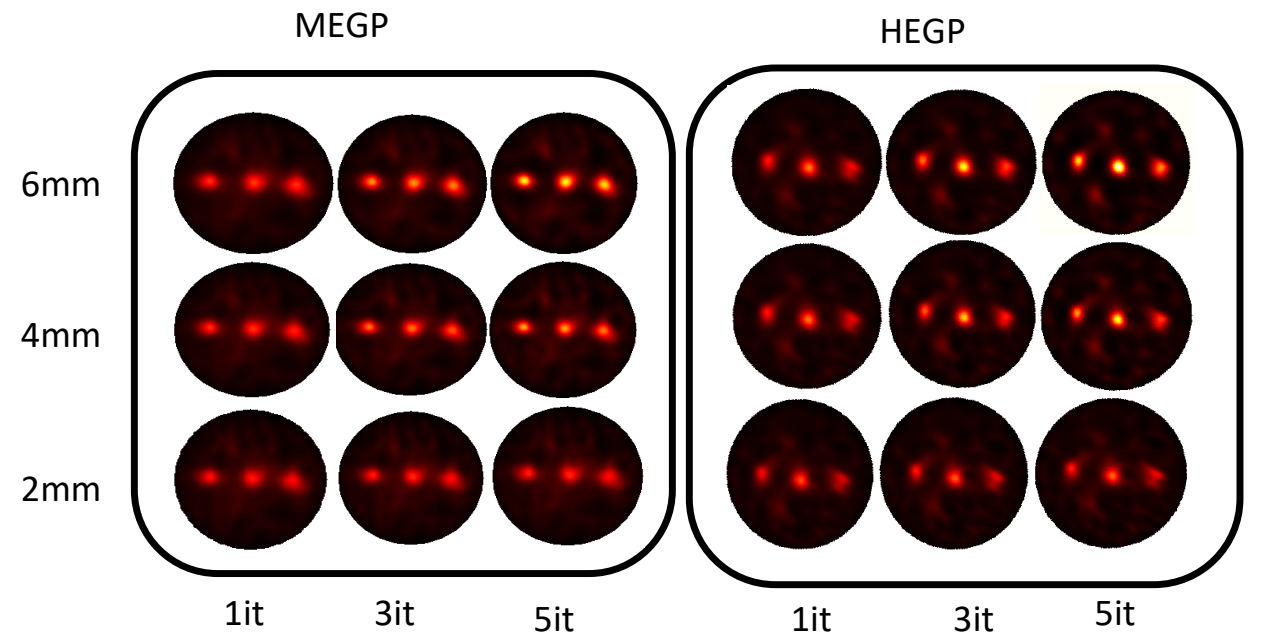

Figure 21: Phantom image qualities for the MEGP and HEGP collimators. Column numbers correspond to the iterations number of the algorithm and rows refer to the FWHM of the PSF.

\subsubsection{MEGP collimator}

The $\mathrm{QH}$ at every iteration for $34 \mathrm{~mm}$ sphere measured based on ROI and VOI method is shown in Fig. 22. The ROI method gave a highest value of $\mathrm{Q}_{\mathrm{H}}$ for the sphere at the fifteenth iteration while the maximum value was found at the sixth iteration for the VOI method. Table 9 shows summary of the results of $\mathrm{Q}_{\mathrm{H}}$ for the sphere sizes using the ROI and VOI methods at the $15^{\text {th }}$ and $6^{\text {th }}$ iterations respectively. 


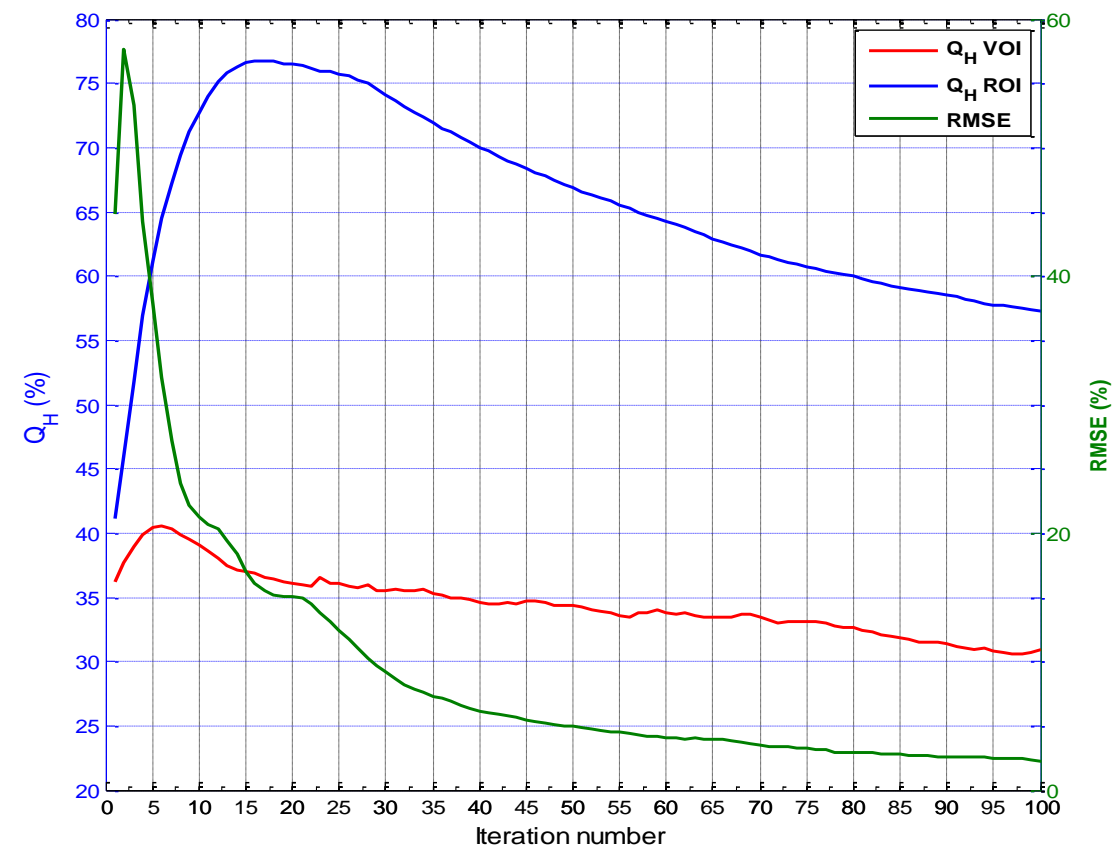

Figure 22: ROI and VOI based contrast resolution coefficient for different iteration number, FWHM $=5 \mathrm{~mm}$ and the RMSE for the $34 \mathrm{~mm}$ sphere.

Table 9: $\mathrm{Q}_{\mathrm{H}}$ calculated using the ROI and VOI method for the sphere sizes

\begin{tabular}{cccc}
\hline $\begin{array}{c}\text { Sphere (diameter, } \\
\mathrm{mm})\end{array}$ & $\mathrm{Q}_{\mathrm{H}}$ before $(\%)$ & $\begin{array}{c}\mathrm{Q}_{\mathrm{H}} \text { after_VOI } \\
(\%)\end{array}$ & $\begin{array}{c}\mathrm{Q}_{\mathrm{H}} \\
\text { after_ROI } \\
(\%)\end{array}$ \\
\hline 34 & 29.9 & 41.0 & 77.0 \\
\hline 31 & 28.1 & 32.0 & 65.0 \\
\hline 25 & 22.2 & 30.0 & 90.0 \\
\hline 16 & 7.6 & 8.0 & 14.0 \\
\hline 12 & 8.7 & 9.0 & 18.0 \\
\hline 10 & -9.1 & -8.0 & 20.0 \\
\hline Mean & 14.6 & 18.7 .0 & 47.0 \\
\hline Std. & 15.0 & 18.60 & 33.9 \\
\hline
\end{tabular}

The optimal iteration number for the subsequent application of the resolution recovery algorithm on patient ${ }^{90} \mathrm{Y}$ microsphere SPEC/CT images was used from the VOIs analyses of the phantom images (Fig. 23). The choice of the VOI method is due to the fact that this approach 
considers the improvement of the total reconstructed count within a volume which is required for accurate dosimetry estimation.
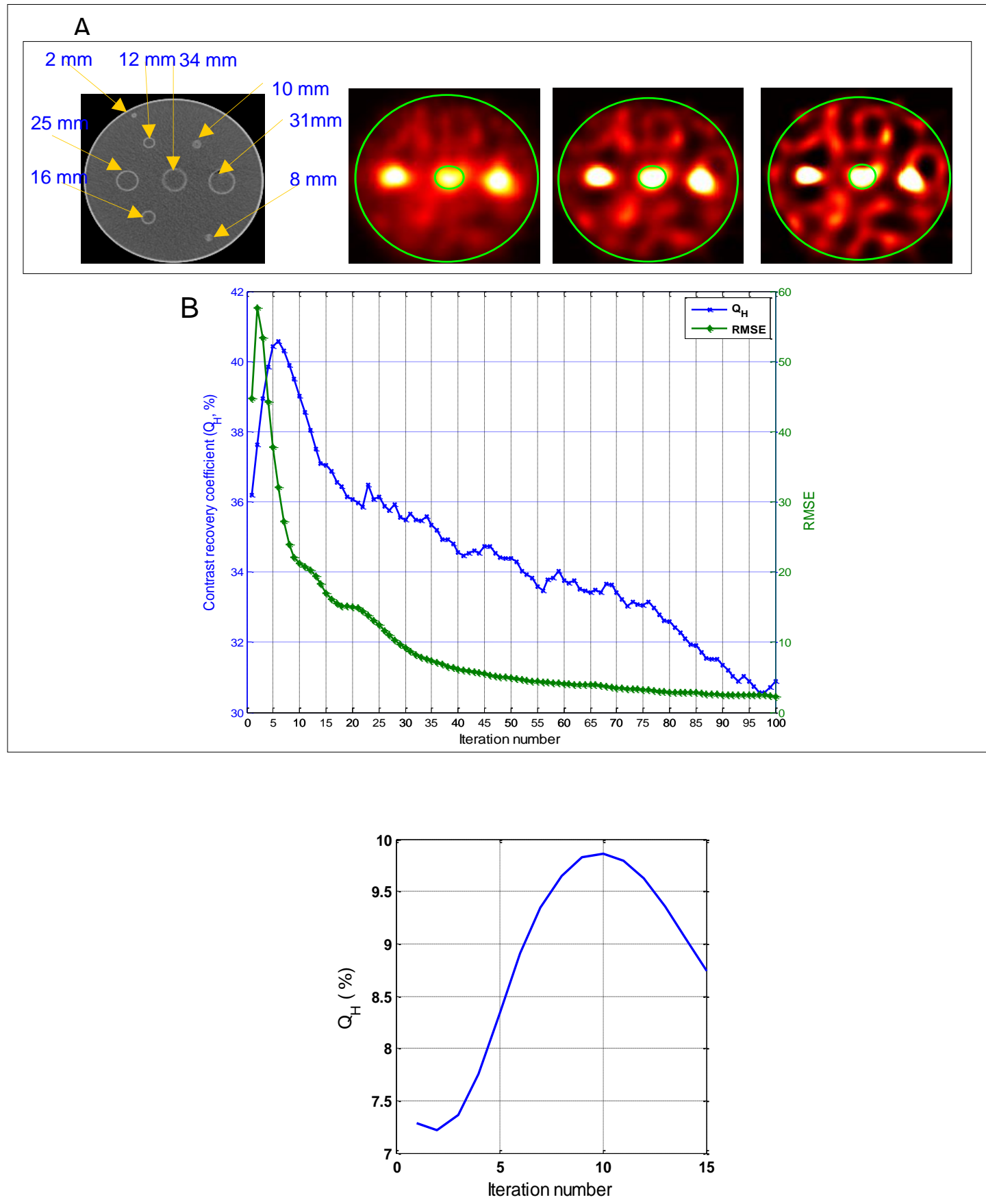

Figure 23: (A) Phantom CT scan and SPECT images for iteration numbers 0, 6 and 15 left to right. (B) Plot of $\mathrm{Q}_{\mathrm{H}}$ (right axis) and RMSE (left axis) vs. iteration number for the $34 \mathrm{~mm}$ sphere (upper) and $\mathrm{Q}_{\mathrm{H}}$ for the $12 \mathrm{~mm}$ sphere peaking at different iteration number (bottom). 
Figure 24 shows examples of improvements in the CNRs between tumor and the background for patient ${ }^{90} \mathrm{Y}$ microsphere bremsstrahlung SPECT/CT images. For the patients shown (Patients 5, 12 and 14), the CNRs were respectively 18.9, 14.3 and 48.8 before and 24.1,21.4 and 51.7 after spatial resolution recovery. For the phantom study, improvement in $\mathrm{Q}_{\mathrm{H}}$ ranged between -8.3 to $41.0 \%$. For the smallest spheres $(2,8$ and $10 \mathrm{~mm})$, no improvement in $\mathrm{Q}_{\mathrm{H}}$ was found. Figure 23 also shows line profiles along the 16 and $12 \mathrm{~mm}$ spheres. From the profiles, it is seen that the signal in the 16 $\mathrm{mm}$ sphere significantly differs from the background but the signal within the $12 \mathrm{~mm}$ sphere and the background are indistinguishable.

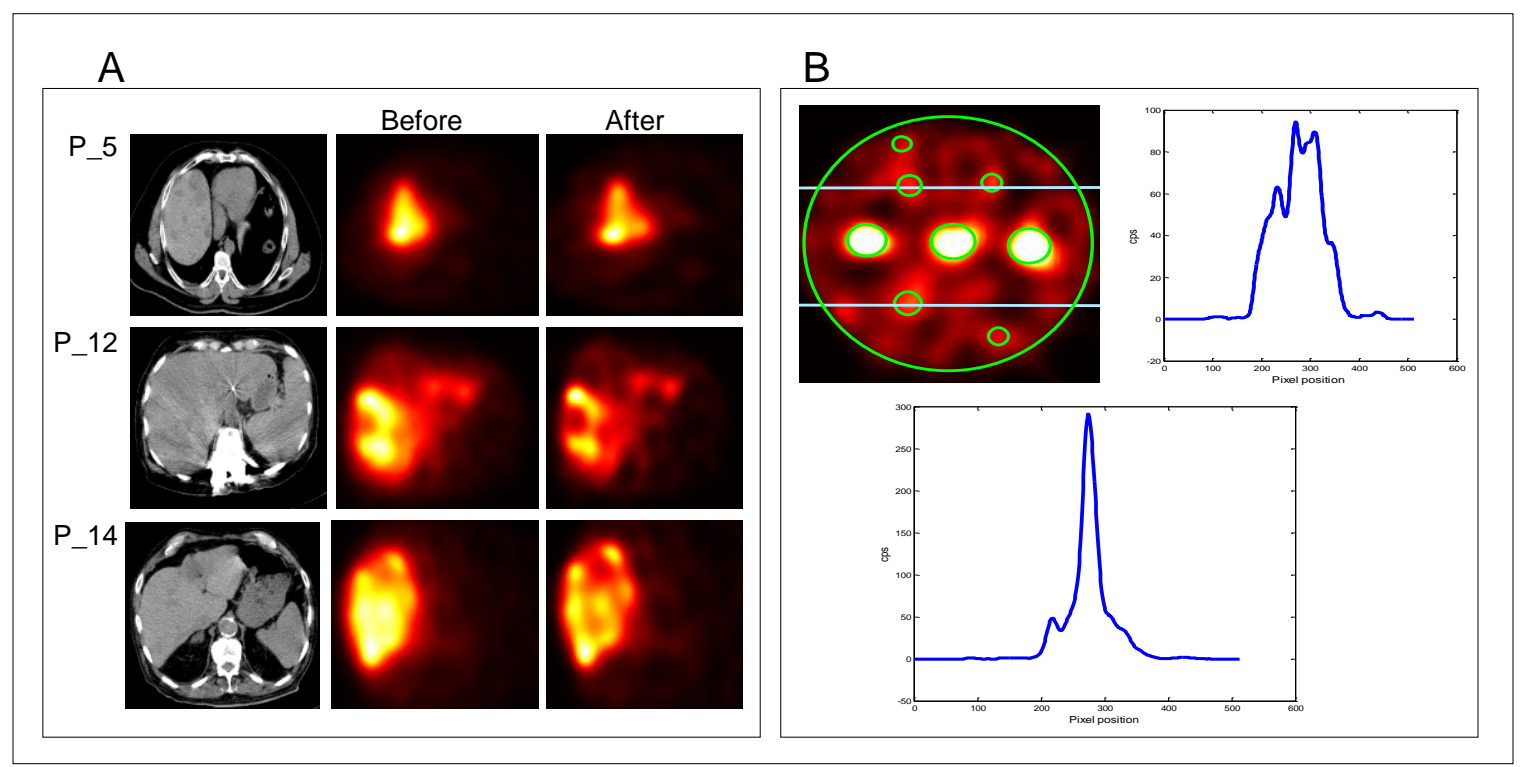

Figure 24: Result of spatial resolution recovery showing the before and after ${ }^{90} \mathrm{Y}$ microsphere bremsstrahlung SPECT images of patients with the respective CT scans. (B) Line profiles through the $16 \mathrm{~mm}$ (lower) and $12 \mathrm{~mm}$ (upper right) spheres of the phantom ${ }^{90} \mathrm{Y}$ bremsstrahlung SPECT/CT image. SPECT images were resized to $512 \times 512$ for display purpose hence higher pixel position for the line profiles.

\subsubsection{HEGP collimator}

For the same FWHM (5mm) as the MEGP collimator, the following result was found for the $34 \mathrm{~mm}$ sphere using the VOI method. 

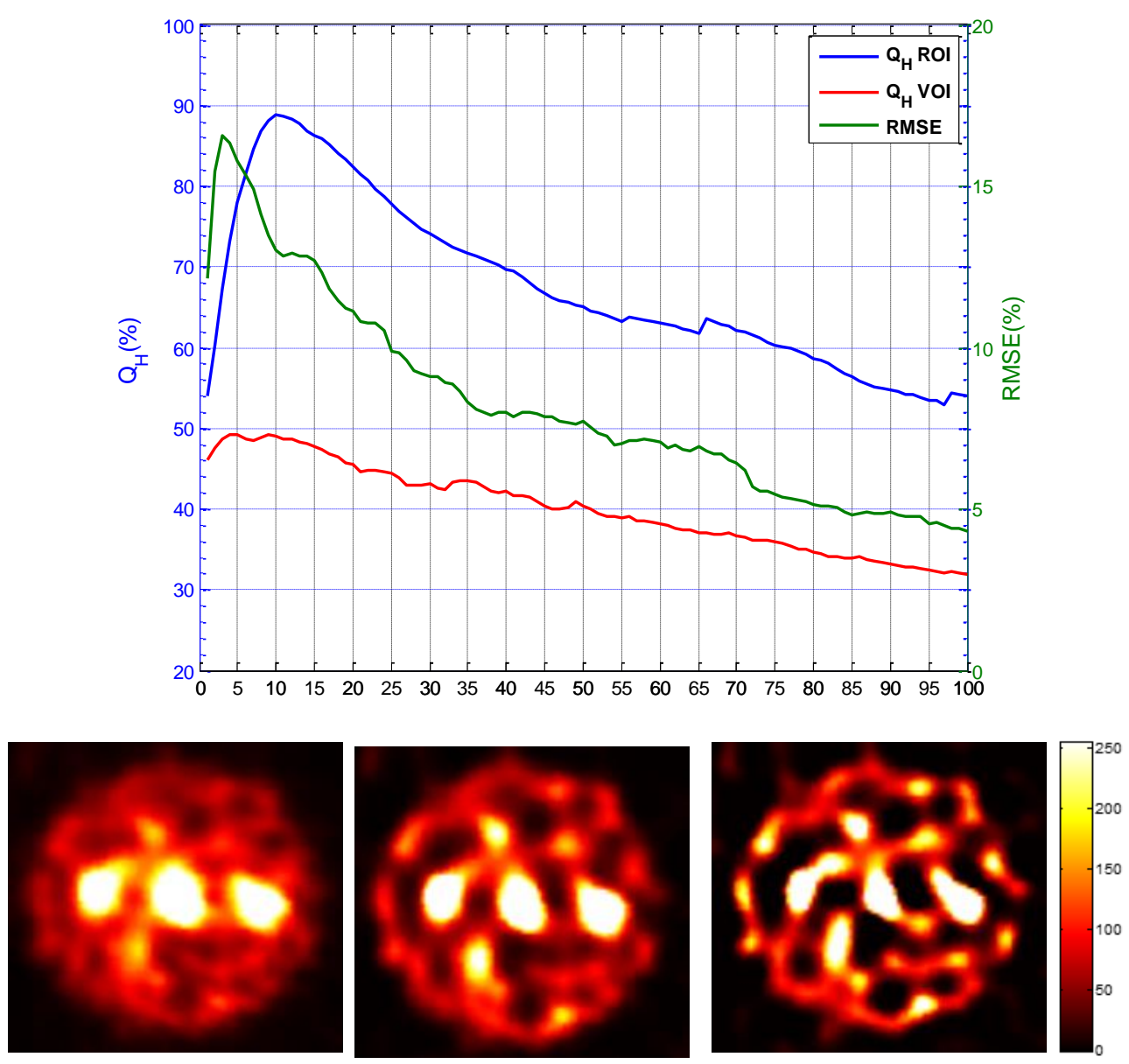

Figure 25: (upper) $\mathrm{Q}_{\mathrm{H}}$ and RMSE with $5 \mathrm{~mm}$ FWHM for the 34-mm sphere by HEGP collimator SPECT/CT imaging and (lower) the before (left) and after images at the fifth (middle) and fifteenth (right) iteration.

Figure 26 summarizes the results of $\mathrm{Q}_{\mathrm{H}}$ for the MEGP and HEGP collimators at the different sphere sizes and variable iteration number. An overall consistent increase in the QH was observed for a FWHM of 5mm both the MEGP and HEGP collimators. Table 10 shows 
ANOVA table for the effect of the choice of collimator and FWHM for the spatial recovery algorithm.
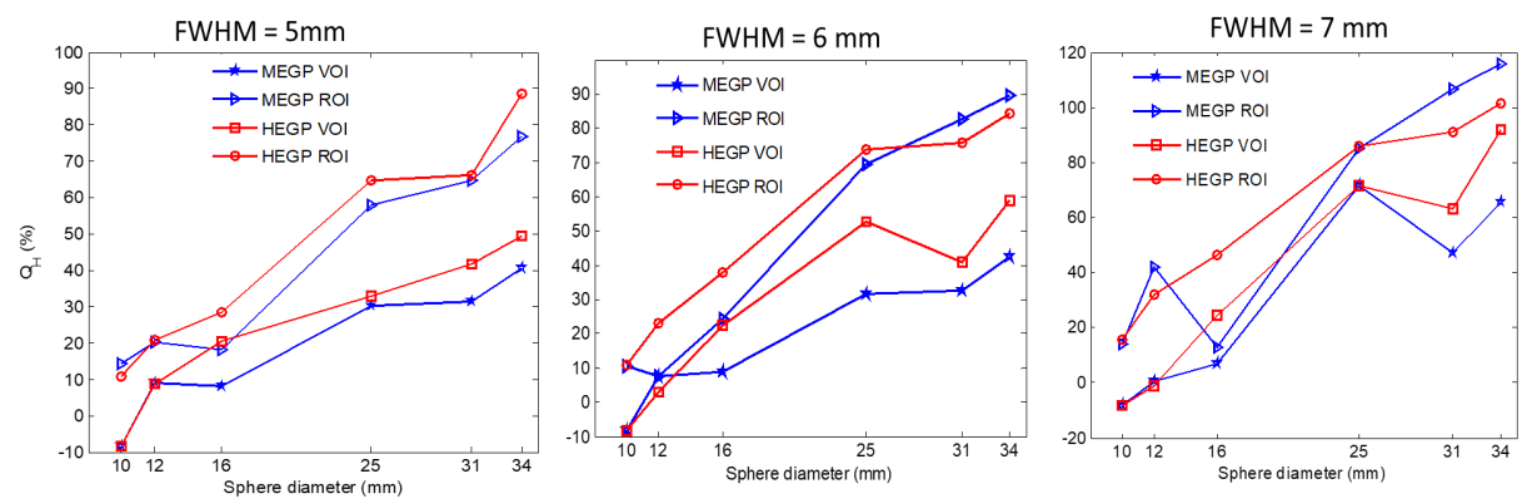

Figure 26: Result of $\mathrm{Q}_{\mathrm{H}}$ for varying FWHM and collimator at different iteration.

\subsubsection{PET/CT study}

For the PET/CT study a significant change in $\mathrm{Q}_{\mathrm{H}}$ was observed with a change in the FWHM (from 5mm to $6 \mathrm{~mm}$ ) of the PSF model. FWHM of $6 \mathrm{~mm}$ was associated with a higher $\mathrm{Q}_{\mathrm{H}}$ of the $34 \mathrm{~mm}$ sphere compared to the SPECT/CT study based on ROI analysis (Fig. 27). The iteration number for the highest $\mathrm{Q}_{\mathrm{H}}$ values however, is similar for both modalities (15 iteration). QH was higher both for the ROI and VOI method for the 30min imaging. 

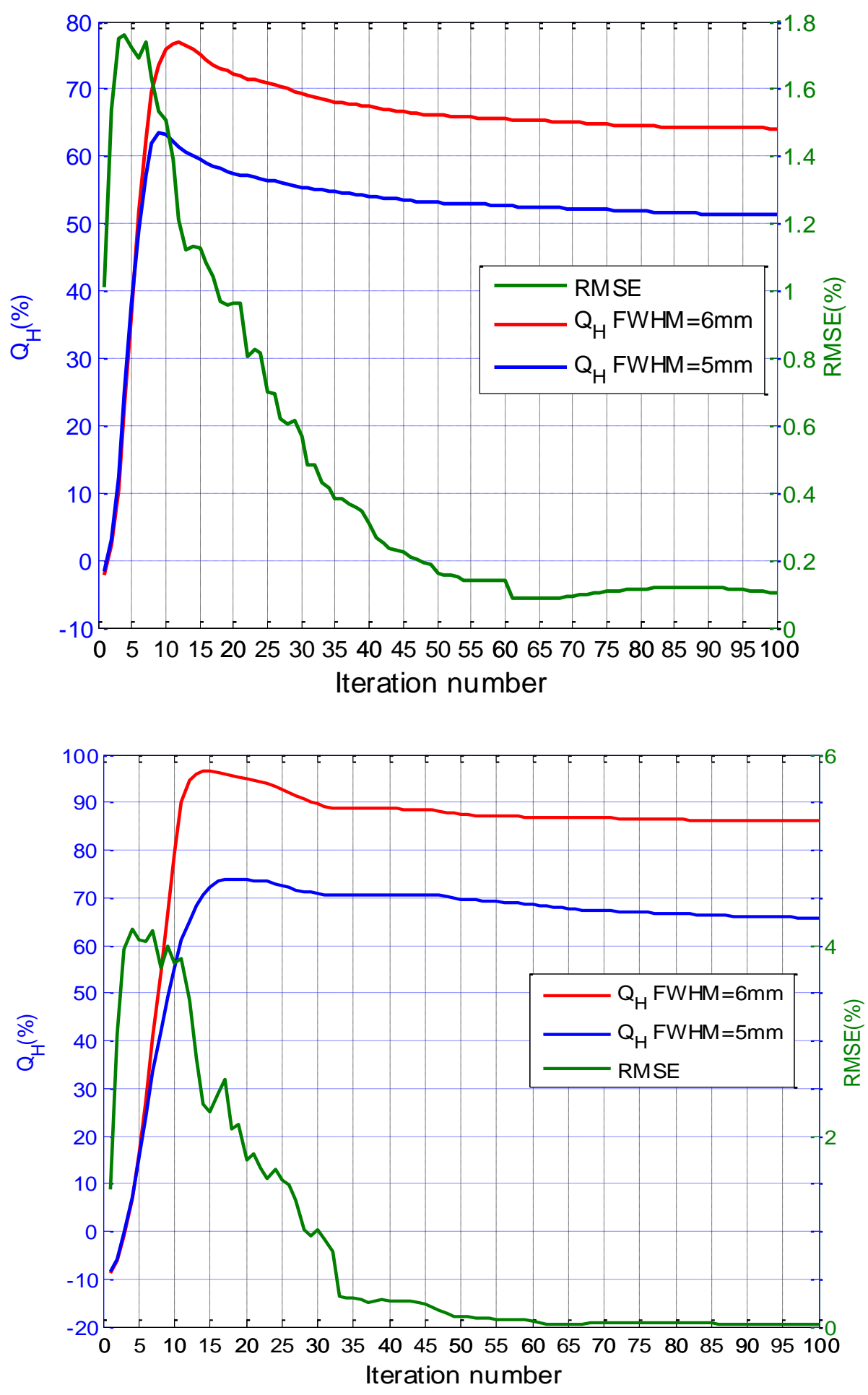

Figure 27: $\mathrm{Q}_{\mathrm{H}}$ (left axis) and RMSE (right axis) with $5 \mathrm{~mm}$ (left) and 6mm (right) FWHM for the $34 \mathrm{~mm}$ sphere by PET/CT TOF imaging of 15 (upper) and 30 (lower) minute imaging. 
The primary objective of this study was to develop a post-reconstruction algorithm to quantitatively improve the ${ }^{90} \mathrm{Y}$ bremsstrahlung imaging using SPECT/CT and PET/CT. The challenges for our method were image degradation due to object scatter, septal penetration and backscatter. Despite the inherent shortcomings, we achieved a meaningful improvement in CNR and $\mathrm{Q}_{\mathrm{H}}$ which were measured in clinical and phantom ${ }^{90} \mathrm{Y}$ bremsstrahlung SPECT/CT images respectively. The small spheres $(2,8$ and $10 \mathrm{~mm})$ showed indifferent results before and after resolution recovery. The challenge to draw the ROIs introduces extra errors in addition to the fact that the volumes might be highly influenced by noise. The smallest volume that gave an acceptable result was the $16 \mathrm{~mm}$ sphere with substantial signal difference from the background. Our result of the highest $\mathrm{Q}_{\mathrm{H}}$ for the $34 \mathrm{~mm}$ sphere was $41 \%$ using a matching VOI. Using similar methods used by other authors to evaluate $\mathrm{Q}_{\mathrm{H}}(17,66)$, that is using an ROI in the slice through the center of the spheres, we found a higher value of $\mathrm{Q}_{\mathrm{H}} \sim 80 \%$ for the $34 \mathrm{~mm}$ sphere. It is worth noting that there are other methods of measuring the background ROI as well. Martí-Climent el al. (56) measured the background from ROIs drawn similar to the sphere diameter of interest throughout the image. The authors studied the $\mathrm{Q}_{\mathrm{H}}$ in ${ }^{90} \mathrm{Y}$ PET/CT study of a Jasczak phantom with six spheres. The sphere VOIs were generated in a similar manner to our method. The authors reported $\mathrm{Q}_{\mathrm{H}}=60 \%$ in a 28 $\mathrm{mm}$ sphere for ${ }^{90} \mathrm{Y}$ PET/CT images reconstructed with OSEM+PSF with TOF described as the optimal method. In our study, the ${ }^{90} \mathrm{Y}$ images reconstructed with OSEM algorithm with TOF PET/CT corrected with post-reconstruction PSF, the result of a closest sphere volume $(25 \mathrm{~mm})$ gave $\mathrm{Q}_{\mathrm{H}}=75 \%$. Although the method used by the authors is feasible, when considering a range of hot concentrations in a warm background that is representative of a microsphere distribution, taking a background measurement as small as the sphere size in different areas that could have no activity might not quite represent the scenario in RMT.

A recent work to correct ${ }^{90} \mathrm{Y}$ bremsstrahlung SPECT/CT images was done by Simen et al. (19). The authors used a technique called background compensation. The technique entails two 
separate imaging of a subject for energy window based scatter correction, but the method highly relies on the selection of energy windows. Energy based scatter correction is different in different makes and models of cameras with different collimator types, thus replication of the method in the clinical environment is impractical. Using this method the authors reported resolution recovery coefficient of $90 \%$ for a $37 \mathrm{~mm}$ sphere in a $10 \mathrm{~mm}$ VOI, much less than the actual sphere VOI.

Our spatial resolution algorithm corrects images for the effect of PSF. The statistical basis of the algorithm makes it suitable for application on nuclear medicine images. In addition, the restoration algorithm is image dependent instead of dependent on the camera make and model through the inclusion of the RMSE criteria between two consecutive iterative images. The restoration algorithm which corrects for the collimator response will also implicitly correct for scatter. This is due to the fact that scatter photons degrade the PSF giving it a long tail especially, in PET camera. Thus the restoration algorithm is capable of improving both the accuracy and precision of the ${ }^{90} \mathrm{Y}$ bremsstrahlung SPECT and PET images.

\subsection{Conclusion}

The proposed spatial resolution algorithm for quantitative image improvement of ${ }^{90} \mathrm{Y}$ bremsstrahlung SPECT/CT and PET/CT gave meaningful results in phantom and patient studies. The method utilizes current clinical ${ }^{90} \mathrm{Y}$ imaging protocols thus can be readily applied in the clinical environment. For the PECT/CT study, however the method can be further tested for different acquisition times and activity concentrations as well as on actual patient ${ }^{90} \mathrm{Y}$ PET/CT images.

\section{Chapter 5: Calibration factor and Activity estimation}

\subsection{Introduction}

In nuclear medicine internal radionuclide dosimetry absolute quantification is a requirement (32). The objective is to provide reconstructed images with each voxel representing the absolute activity concentration in the corresponding region in the patient. Absolute activity 
concentration has many uses in nuclear medicine, such as patient-specific dosimetry in radiotherapy treatment planning and monitoring, tumor classification, and detection of vessel diseases in cardiac imaging. This is achieved by conversion of voxel counts per second (cps) values to activity concentration $(\mathrm{Bq})$ which requires determining the camera calibration factor (sensitivity). The most reliable method to determine the calibration factor $(\mathrm{cps} / \mathrm{Bq})$ of a detector is to perform an experiment such as a point source with a known activity concentration, $\mathrm{A}(\mathrm{Bq} / \mathrm{ml})$, of the radionuclide of interest in a setting with minimal image degrading effects $(15,32)$. The calibration factor is then determined by dividing the total reconstructed counts, $\mathrm{C}$ (counts/per voxel/sec), within the volume of interest by the known radionuclide activity. In PET though, the fraction of the decay, i.e. the branching fraction (B.F.), that occur via positron emission for the radionuclide of interest should be included in the calculation of the calibration factor (11). This is due to the fact that most radionuclides used in PET imaging don't decay by $100 \%$ positron emission, thus the CF in PET is given as:

$$
C F=\frac{C\left(\frac{\text { counts }}{\text { voxel } \times \text { sec }}\right)}{A\left(\frac{B q}{m l}\right) \times B . F .}
$$

Applying a calibration factor calculated using a point source to a population of humans who exhibit variability in terms of anatomy and biokinetics introduces error (67). The error is exacerbated in bremsstrahlung imaging due to attenuation, scatter and collimator-detector effects. In addition, the $\mathrm{CF}$ of ${ }^{90} \mathrm{Y}$ imaging in PET depends on the activity concentration inside a volume rather than total activity (18). As a result, mostly, phantom studies more representative of humans are used for estimating a calibration factor in ${ }^{90} \mathrm{Y}$ imaging. Even using a phantom (such as the IEC body phantom) for the calibration study has shown a substantial bias as it doesn't accurately represent clinical imaging conditions in terms of bremsstrahlung photon attenuation and scatter (19). A direct solution that represents the clinical scenario, i.e. using clinical studies or animal studies, has been suggested for calculation of a calibration factor for absolute quantification (67). 
Animal studies, however, don't quite represent humans in terms of size, the biokinetics and geometric size of the organ uptake that affect the attenuation and scattering of photons. Human studies on the other hand require obtaining sufficient numbers of patients under a similar acquisition protocol. Thus solving for calibration factor that address the unique condition in ${ }^{90} \mathrm{Y}$ imaging under a clinically relevant condition to be able to provide the clinical user with absolute quantitative values is an active research area. In the present chapter, we developed and evaluated an approach to quantitate ${ }^{90} \mathrm{Y}$ imaging. The goal is to assure clinical practicability and to establish a baseline for image based calibration factor generation.

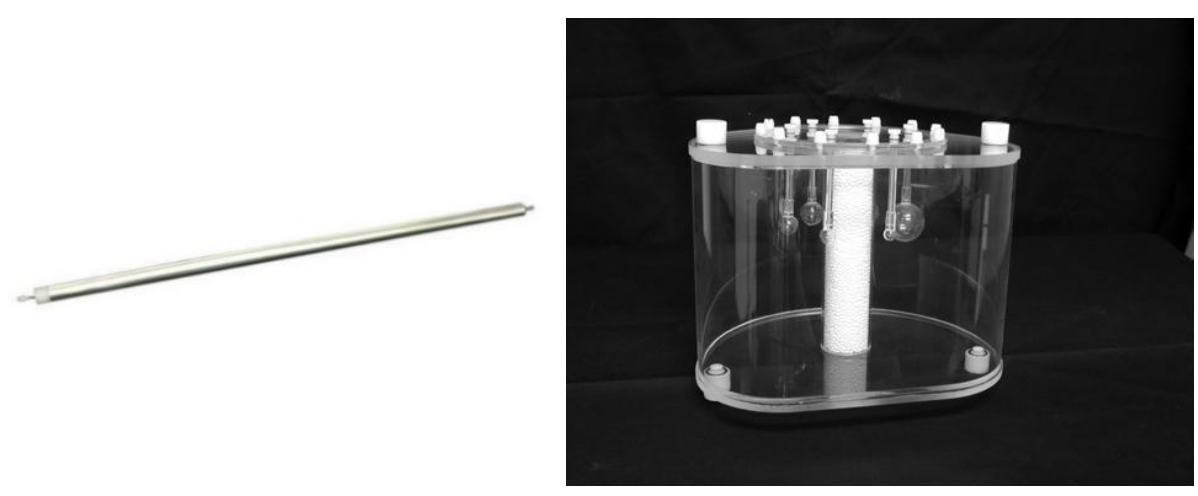

Figure 28: (left) Rod phantom and (right) IEC body phantom used in calibration factor study (68).

\subsection{Materials and Methods}

\subsubsection{Calibration Factor}

In order to obtain absolute quantification values from reconstructed SPECT and PET images, calibration of the imaging system is necessary. We propose two methods to derive absolute activity quantification in MBq from counts of the reconstructed images. For the SPECT/CT study calculation the calibration factor was done from the spatially recovered ${ }^{90} \mathrm{Y}$ microsphere SPECT/CT of the 17 patients. The first approach (method 1) uses the entire total reconstructed counts within the field of view (FOV) of the SPECT detector (Fig. 29). The second approach (method 2) used counts from the liver segments only. In this method, the liver was first segmented as described in the image processing section and total counts within the liver VOIs were taken. This 
method avoids counts due to image artifacts. Calibration curves were generated using linear regression analysis to derive the relationship between patient administered dose and reconstructed counts. The CFs were calculated from the slopes of the calibration curves.

For the phantom study, the total reconstructed counts within the phantom boundary were considered. The total activity for the phantom was the sum of the activities inside the spheres and the background. The CF from the phantom study was evaluated to be within the $95 \%$ confidence interval (CI) of the calibration curves from the ${ }^{90} \mathrm{Y}$ microsphere SPEC/TCT patient studies to validate its application on the phantom images. For the PET/CT phantom study, the activity inside the phantom was adjusted for a B.F of $0.003 \%$ as explained earlier.

Since we sought to investigate the distribution between pre and post-treatment images, we also calculated calibration factor for the ${ }^{99 \mathrm{~m}} \mathrm{Tc}-\mathrm{MAA}$ imaging using a line source both in air and water as shown in Fig. 30.

The calibration factor (CF) for all the phantom studies was defined as in Eqn. 26,

$$
C F=\frac{\sum_{j \in V O I} f_{j}}{A_{c o r r}}
$$

where $f_{j}$ is the reconstructed counts from the corrected images in the $j$ th voxel that belong to the defined VOIs, and the $A_{\text {corr }}$ is the total true administered activity corrected for decay from $\mathrm{A}_{0}$ measured at the time of activity calibration $\mathrm{t}_{0}$ (assumed to be 0 ) to the start time of image acquisition $t_{i}$ using a decay constant $(\lambda)$ given as,

$$
\begin{aligned}
& A_{\text {corr }}=A_{0} e^{-\lambda\left(t_{i}-t_{0}\right)} \\
\lambda= & \frac{\ln 2}{T_{1 / 2}},
\end{aligned}
$$


where $\mathrm{T}_{1 / 2}$ is the half-life of ${ }^{90} \mathrm{Y}$, which is $64.0 \mathrm{hr}$. (43). Since the half-life of ${ }^{90} \mathrm{Y}$ is much longer than the image acquisition time, the radioactive decay that occurs during the acquisition is not corrected. The decay corrected calibration factor in the VOI is then,

$$
C F=e^{-\lambda\left(t_{i}\right)} \times \frac{\sum_{j \in V O I} f_{j}}{A_{0}}
$$

The precision of the calibration factor could of course be impacted by the dose calibrator measurement ( $\pm 2 \%$ as specified by the manufacturer), experimental error such as activity and volume measurement and statistical variations of the measured counts. Patient administered activities were also corrected for decay with an average time of $t_{i}=2 \mathrm{hr}$. which is the time between when the injected activity is calibrated and patient imaging. There is also a discrepancy here due to the logistics of the clinical setup where the time of activity calibration and actual administration to the patient might have been recorded as similar events.

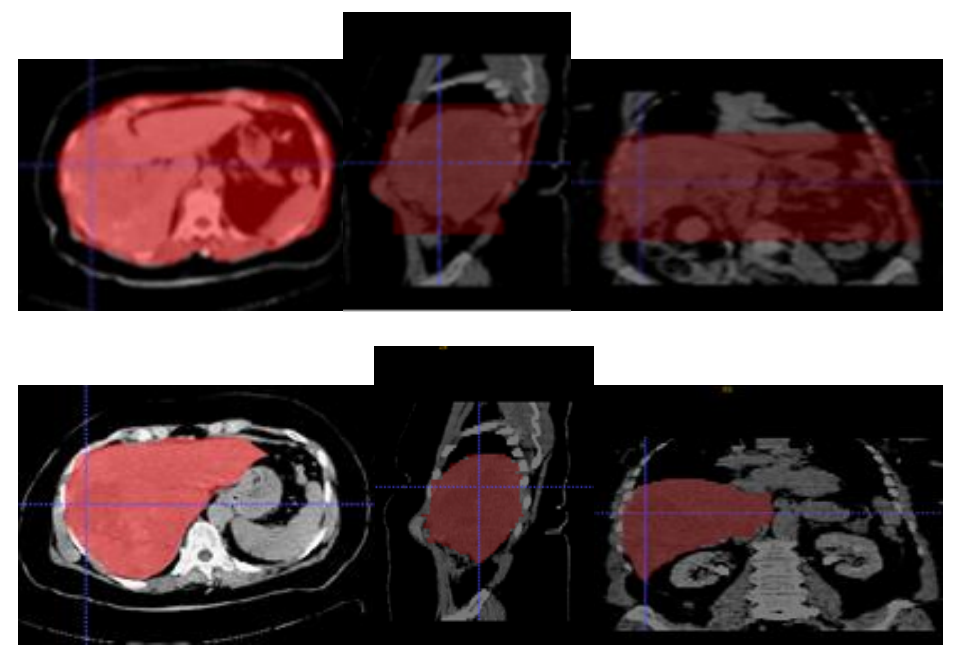

Figure 29: Anatomical representation (transverse, coronal and sagittal view) of method 1 (upper) and method 2 (lower) of calculating the CFs for the patient study. 

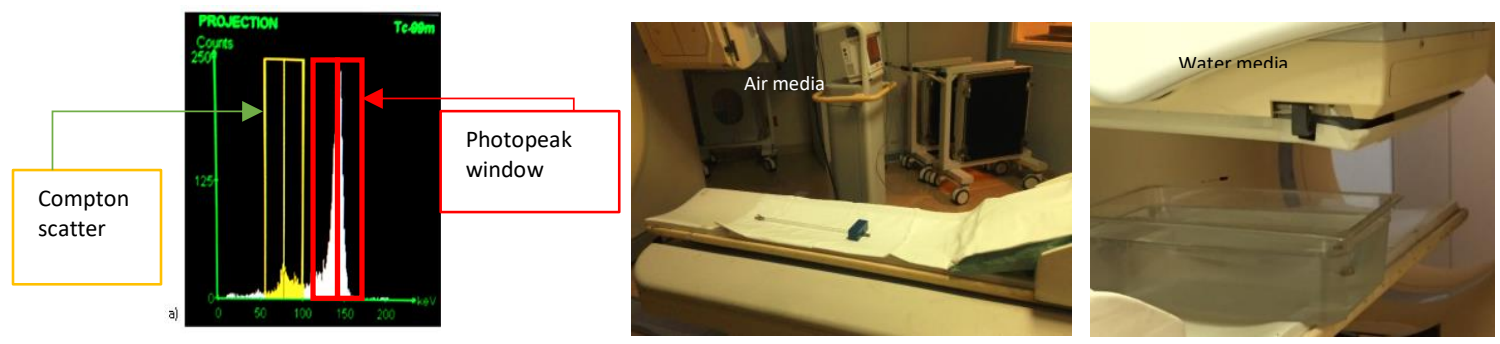

Figure 30: SPECT/CT imaging window of ${ }^{99 \mathrm{~m}} \mathrm{Tc}$ (left), and line source filled with ${ }^{99 \mathrm{~m}} \mathrm{Tc}$ solution in air (middle) and water media (right).

\subsubsection{Activity estimation}

The activity of ${ }^{90} \mathrm{Y}$ at a reconstructed pixel and in a defined volume was given by Eqn. 29 and 30 respectively. For the patient studies, total liver activity estimation was compared to the true administered dose. For the phantom study, activities inside the eight spherical inserts were compared to the activities measured using the dose calibrator. The relative percent error between true and estimated activity was determined by Eqn. 31 .

$$
\begin{aligned}
& A_{i, j, k}=\frac{f_{i, j, k}}{C F} \\
& A_{V}=\frac{\sum_{i, j, k \in V O I} f_{i, j, k}}{C F} \\
& \% \text { Error }=\frac{A_{\text {corr }}-A_{V}}{A_{\text {corr }}} \times 100
\end{aligned}
$$

\subsection{Results and Discussion}

\subsubsection{Calibration factor}

Results of the regression analyses (Fig. 31) of the relationships between cps and administered activity gave the following results for the patient study, the slopes being the CFs (cps/MBq); method $1, \mathrm{CF}_{1}=20929 \mathrm{cps} / \mathrm{MBq}$ with $95 \%$ CI $16,281 \leq \beta \leq 25,576 \mathrm{cps} / \mathrm{MBq}\left(\mathrm{R}^{2}=\right.$ 0.86 and standard error $(\mathrm{SE})=2181)$ and method $2, \mathrm{CF}_{2}=8389 \mathrm{cps} / \mathrm{MBq}$ with $95 \% \mathrm{CI}$ of 7050.7 $\leq \beta \leq 9729.3\left(\mathrm{R}^{2}=0.92\right.$ and $\left.\mathrm{SE}=628\right)$. The $\mathrm{CF}$ from the phantom SPECT/CT study is 9049 cps/MBq, which doesn't fall within $95 \% \mathrm{CI}$ of $\mathrm{CF}_{1}$ but does fall within the $95 \% \mathrm{CI}$ of $\mathrm{CF}_{2}$. 
The HEGP collimator imaging gave a $\mathrm{CF}=14,990$ and 15,086 cps/MBq for FWHM of 5 and $6 \mathrm{~mm}$ respectively. For the PET/CT phantom study, the measured CF of the camera was $6844 \mathrm{cps} / \mathrm{MBq}$.

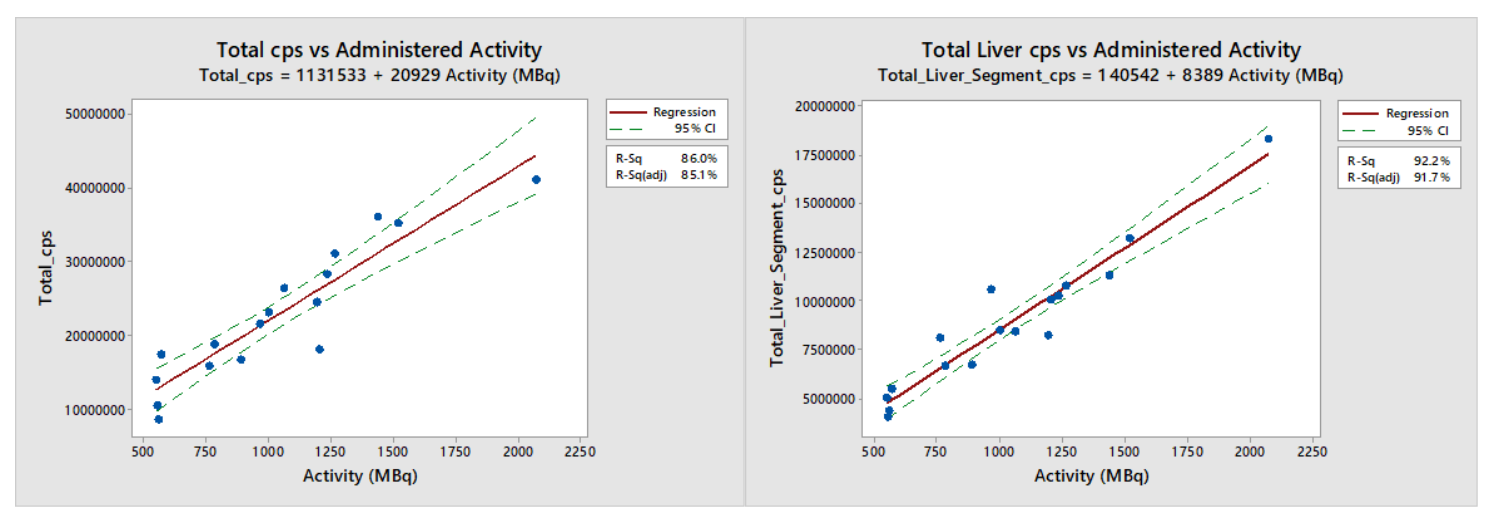

Figure 31:Linear regression plots of the calibration curves with the 95\% CI range (green dashed lines) for method 1 (left) and method 2 (right).

\subsubsection{Activity estimation}

\subsubsection{Patient Study}

The total activity inside the liver was estimated for each patient using the CFs derived from the two methods. For $\mathrm{CF}_{1}$, total liver activity estimation resulted in mean percent error of $59 \pm 5 \%$. Applying $\mathrm{CF}_{2}$ gave the smallest error $(-4 \pm 12 \%)$, thus it was used for subsequent analysis. Table 9 shows results of total activity estimation within the liver VOIs using $\mathrm{CF}_{2}$. For 12 out of 17 patients, the estimated total liver activity percent errors were within $\pm 10 \%$ giving overall satisfactory results. As our study is retrospective based on anonymized data, we couldn't provide possible clinical reasons for the larger deviations of the estimated activities. 
Table 10: Administered activities and total estimated activities inside liver VOI. Administered Activity Estimated Activity

$\begin{array}{llll}\text { Patient No } & (\mathrm{MBq}) & (\mathrm{MBq}) & \text { Error }(\%)\end{array}$

\begin{tabular}{cccc}
\hline Pat_1 & 548 & 598 & -9 \\
\hline Pat_2 & 555 & 480 & 14 \\
\hline Pat_3 & 559 & 523 & 6 \\
\hline Pat_4 & 570 & 652 & -14 \\
\hline
\end{tabular}

\begin{tabular}{cccc}
\hline Pat_5 & 759 & 967 & -27 \\
\hline Pat_6 & 781 & 788 & -1
\end{tabular}

\begin{tabular}{cccc}
\hline Pat_7 & 888 & 800 & 10 \\
\hline Pat_8 & 966 & 1251 & -30
\end{tabular}

\begin{tabular}{cccc}
\hline Pat_9 & 999 & 1009 & -1 \\
\hline Pat_10 & 1062 & 1003 & 6
\end{tabular}

\begin{tabular}{cccc}
\hline Pat_11 & 1203 & 1202 & 0 \\
\hline Pat_12 & 1195 & 1323 & -11 \\
\hline
\end{tabular}

\begin{tabular}{cccc}
\hline Pat_13 & 1236 & 1221 & 1 \\
\hline Pat_14 & 1262 & 1288 & -2
\end{tabular}

\begin{tabular}{cccc}
\hline Pat_15 & 1436 & 1350 & 6 \\
\hline Pat_16 & 1517 & 1572 & -4
\end{tabular}

\begin{tabular}{cccc}
\hline Pat_17 & 2072 & 2188 & -6 \\
\hline Mean & 1036 & 1071 & -4 \\
\hline Std. & 410 & 432 & 12 \\
\hline
\end{tabular}




\subsubsection{Phantom Study}

Table 11 shows results of activity estimates inside the spheres and background for the phantom study with total mean percent error of $-23 \pm 41 \%$ for the MEGP collimator. Table 12 shows ANOVA study that summarizes the effect of the choice of collimator and FWHM for the spatial resolution recovery on accurate activity estimation. The result shows that for the specific acidity used in our study, collimator choice is insignificant $(p=0.17)$ whereas the FWHM plays is an important factor for accurate activity estimation.

Table 11: Phantom true and estimated activities.

\begin{tabular}{|c|c|c|c|c|c|c|c|}
\hline \multirow{2}{*}{$\begin{array}{l}\text { Diameter } \\
(\mathrm{mm})\end{array}$} & \multirow{2}{*}{$\begin{array}{l}\text { True } \\
\text { Activity } \\
(\mathrm{MBq})\end{array}$} & \multicolumn{3}{|c|}{ Estimated Activity (MBq) } & \multicolumn{3}{|c|}{$\%$ Error } \\
\hline & & MEGP & HEGP@5 & HEGP@6 & MEGP & HEGP@5 & HEGP@6 \\
\hline 34 & 11.10 & 11.55 & 9.23 & 9.55 & -4.05 & 16.88 & 13.95 \\
\hline 31 & 8.88 & 9.16 & 8.40 & 8.67 & -3.15 & 5.35 & 2.31 \\
\hline 25 & 4.44 & 4.50 & 4.31 & 4.48 & -1.35 & 2.87 & -0.82 \\
\hline 16 & 1.11 & 1.17 & 1.21 & 1.23 & -5.41 & -8.84 & -10.39 \\
\hline 12 & 0.56 & 0.62 & 0.61 & 0.63 & -10.71 & -10.79 & -13.80 \\
\hline 10 & 0.28 & 0.36 & 0.48 & 0.47 & -28.57 & -71.35 & -67.86 \\
\hline 8 & 0.14 & 0.31 & 0.00 & 0.00 & -121.47 & 100.00 & 100.00 \\
\hline $6 \times 10^{3} \mathrm{ml}$ & 255.30 & 282.93 & 183.69 & 183.32 & -10.82 & 28.05 & 28.19 \\
\hline Mean & 35.23 & 38.83 & 25.99 & 26.04 & -23.19 & 7.77 & 6.45 \\
\hline Std. & 89.02 & 98.73 & 59.70 & 59.55 & 40.62 & 47.68 & 47.16 \\
\hline
\end{tabular}


Table 12: ANOVA for the choice of collimator and FWHM

$\begin{array}{lrrrrr}\text { Analysis of Variance } & & & \\ \text { Source } & \text { DF } & \text { Adj SS } & \text { Adj MS } & \text { F-Value } & \text { P-Value } \\ \quad \text { Collimator } & 2 & 1.0337 & 0.5169 & 4.87 & 0.170 \\ \quad \text { FWHM } & 1 & 7.0064 & 7.0064 & 65.98 & 0.015 \\ \text { Error } & 2 & 0.2124 & 0.1062 & & \\ \text { Total } & 5 & 8.2525 & & & \end{array}$

With the clinical available reconstruction method and application of spatial resolution recovery algorithm, total liver activity estimation gave percent error of $-4 \pm 12$ using the CF found from counts within the liver VOIs with the MEGP collimator. Siman et al. (19) generated a global CF derived from patient studies where entire counts within the FOV were considered. The global CF was applied to an IEC phantom with a $37 \mathrm{~mm}$ sphere insert and the authors reported an error of $-25 \%$ with respect to the true activity. For our phantom study, we used CF estimated from the total reconstructed counts, which fall within $95 \%$ CI of the patient calibration curve, and error as low $1 \%$ in the $25 \mathrm{~mm}$ sphere was achieved with total mean percent error $-23 \pm 41 \%$. Sphere volumes $\leq$ $12 \mathrm{~mm}$ resulted in the highest error.

The HEGP collimator study gave total mean percent error of $7.77 \pm 48 \%$ and $6.45 \pm 47 \%$ with FWHM of 5 and $6 \mathrm{~mm}$ respectively. The overall calculated error is much less for HEGP compared to the MEGP collimator study estimated activity values. The activity inside the smaller volumes $(8 \& 10 \mathrm{~mm})$ is overestimated in the HEGP collimator although the volumes showed an improved resolution compared to the MEGP collimator. The fact that attenuation and scatter correction of the vendor is not optimized for the HEGP collimator at the image acquisition window $(150 \pm 30 \%)$ might have contributed the higher discrepancy of activity estimation. For specified SPECT acquisition window, the CT transmission imaging should have to be centered at $140 \mathrm{keV}$ to correct for the attenuation. Due to technical difficulty this wasn't achieved in our HEGP collimator study. 
The PET/CT study measurement in areas of high activity, such as the 34 and $25 \mathrm{~mm}$ spheres, gave errors of $-1.1 \%$ and $6.1 \%$ respectively for the 30 min imaging. For estimates in value regions of low activity, such as the $12 \mathrm{~mm}$ sphere, error in the order of $57 \%$ was observed. The ${ }^{90} \mathrm{Y}$ PET imaging gave a more accurate estimation of the true activity in areas of high activity compared to ${ }^{90} \mathrm{Y}$ bremsstrahlung SPECT/CT imaging. However, the SPECT imaging gave a much lesser error in areas of low activity compared to similar measurement in PET.

\subsection{Conclusion}

Approaches for calculating image based $\mathrm{CF}$ were evaluated. For the ${ }^{90} \mathrm{Y}$ microsphere

patient study, CF generated counts from the liver VOIs gave a much more accurate result compared to a CF generated from counts within the entire FOV of the SPECT/CT camera. In the phantom SPECT/CT study, the HEGP collimator gave an overall lower error of activity estimation however, it resulted in higher discrepancy in the smaller sphere volumes. It is observed that SPECT images give better results in low activity areas than PET images. But the two images give comparable results in high activity areas.

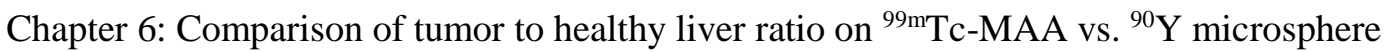

\section{SPECT/CT}

\subsection{Introduction}

The protocol of RMT with ${ }^{90} \mathrm{Y}$ involves a prior liver perfusion scan with ${ }^{99 \mathrm{~m}} \mathrm{Tc}-\mathrm{MAA}$ using planar and SPECT/CT imaging. This prior assessment serves as a surrogate of microsphere distribution to assess lung shunting, endovascular mapping, extrahepatic deposition, to predict absorbed dose in target volumes and to select the catheter position for tumoral targeting (69). Various authors have investigated the predicted dosimetry with the actual ${ }^{90} \mathrm{Y}$ microsphere distribution in SPECT/CT or PET/CT imaging (10,69-71). Accurate tumor and healthy liver predictive dosimetry is essential for patient safety, and evaluation of dose response (10). But a 
quantitative uptake analysis of tumor and nontumoral liver requires a precise calculation of the vascularized volume. Generally, this calculation is done on CT which is relatively easy. But in cases where there is anatomical variation, such as when multiple distinct arterial branches vascularize the liver, CT based functional liver volume calculation is problematic (30). In this case, depending on the radionuclide of interest, SPECT or PET is used for calculating the functional liver volume vascularized by each separate arterial branch. In most studies though, that compared ${ }^{99 \mathrm{~m}} \mathrm{Tc}-$ MAA and ${ }^{90} \mathrm{Y}$ microsphere uptake distribution, tumor areas were delineated on the CT scans. In addition, the two images were evaluated separately, i.e. separate tumor segmentation was done on the CT scans of ${ }^{99 \mathrm{~m}} \mathrm{Tc}-\mathrm{MAA}$ and ${ }^{90} \mathrm{Y}$ microspheres (70-72). The method of CT based tumor delineation is cumbersome for large data sets with multiple tumors per patient requiring a repetitive task on the separate ${ }^{99 \mathrm{~m}} \mathrm{Tc}-\mathrm{MAA}$ and ${ }^{90} \mathrm{Y}$-microsphere images. In addition $\mathrm{CT}$ based tumor delineation will take into account cold areas within the tumor, such as necrosis, which will overestimate tumor volume but underestimate tumor dose (69). Thus, a more reliable method of tumor delineation for dosimetry purpose and a less cumbersome method that avoids a repetitive task is essential in order to compare the ${ }^{99 \mathrm{~m}} \mathrm{Tc}-\mathrm{MAA}$ and ${ }^{90} \mathrm{Y}$ microsphere distributions.

\subsection{Materials and Methods}

\subsubsection{Co-registration of ${ }^{99 \mathrm{~m} T c-M A A ~ v s . ~}{ }^{90} \mathrm{Y}$ SPECT}

To compare the correlation between uptake distribution on ${ }^{99 \mathrm{~m} T c-M A A}$ and ${ }^{90} \mathrm{Y}$ microsphere SPECT/CT images, the two images were co-registered as the two SPECT/CT images have different number slices and voxel sizes (Fig. 32). Image registration involves estimating a mapping between a pair of images. One image is assumed to remain stationary (the reference image), whereas the other (the source image) is spatially transformed to match the stationary image.

We have tried two approaches; taking the CT images as a reference ("fixed image") and the SPECT images as a source ("moving image"). In the second method, ${ }^{90} \mathrm{Y}$ microsphere SPECT 
was taken as the reference image and the ${ }^{99 \mathrm{~m}} \mathrm{Tc}-\mathrm{MAA}$ SPECT and ${ }^{90} \mathrm{Y}$ CT images provided the source images. The latter method was tested to preserve the counts in the ${ }^{90} \mathrm{Y}$ microsphere SPECT images from being inter and/or extrapolated by the registration method.
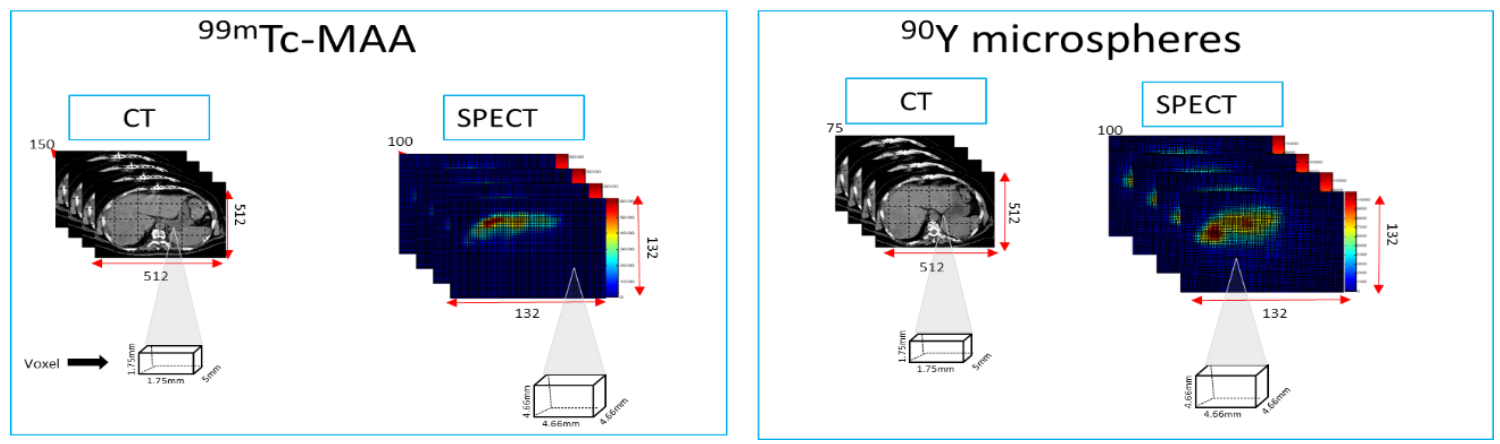

Figure 32: Variation in the number of slices and voxel sized between the CT and SPECT slices between the pre and post-treatment images.

Two image registration tools were used, FMRIB's Software Library (FSL) and Statistical Parametric Mapping (SPM), both well-established tools extensively used in neural imaging for registering anatomical and functional images (73). We found visually best results using SPM thus the following explanation will focus on the details of SPM.

\section{Statistical Parametric Mapping Registration}

Statistical parametric mapping (SPM) is used to identify regionally specific effects in imaging data and is a suitable approach for characterizing functional anatomy and tumor related changes (73). SPM is a voxel-based approach that employs topological inference. The method entails the construction of continuous statistical processes to test hypotheses about regionally specific effects (73). The statistical parametric maps are image values that are distributed according to a probability density function under the null hypothesis, usually the Student's t or F-distribution. Statistical analysis of image data corresponds to inverting generative models of the data to partition observed responses into components of interest, confounds and error. Inferences are then pursued using statistics that compare interesting effects and the error, i.e. each and every voxel is analyzed 
using statistical test (73). The resulting statistical parameters are then assembled into an image which are continuous statistical processes of random fields. Random fields model the univariate probabilistic characteristics of an image and non-stationary spatial covariance structure.

Various tools in the SPM software has been tested. The most relevant to our work were: Rigid body registration and Non-linear registration. Rigid body registration is one of the simplest forms of image registration commonly used for registering within modality or different modality images of a single subject. Images are aligned by finding the rotations and translations that optimize some mutual function of the images. However, rigid body registration is limited to optimizing differences of the images due to subject movement.

Our research question not only addressed subject movement but also changes in the shape of functional volumes from image distortion due to image degrading factors explained in the SPECT/CT imaging section above. These distortions can result in a significant signal accumulation over numerous scans. Non-linear registration addresses differences purely due to image artefacts, as well as interaction due to image distortion, and movement of the subject are considered in nonlinear image registration. The spatial normalization function of SPM12 uses this principle, and hence is applied in this work. To choose the most appropriate approach, that is, using either the CT or the ${ }^{90} \mathrm{Y}$ SPECT as the reference image (Fig. 33), we calculated the mutual information between the co-registered ${ }^{90} \mathrm{Y}$ microsphere and ${ }^{99 \mathrm{~m} T c-M A A}$ SPECT images from the two methods. The mutual information between the two images is defined as:

$$
\begin{array}{cc}
I(A, B)=H(A)+H(B)-H(A, B) & 32 \\
H(A)=-\sum_{a} p_{A}(a) \log p_{A}(a) & 33 \\
H(B)=-\sum_{b} p_{B}(b) \log p_{B}(b) & 34 \\
H(A, B)=-\sum_{a, b} p_{A, B}(a, b) \log p_{A, B}(a, b) & 35
\end{array}
$$


$H(A)$ and $H(B)$ are the entropy of images $A$ and $B$ respectively, and $H(A, B)$ is their joint entropy, $p_{A}$ and $p_{B}$ are probability distributions (histogram) of image $A\left({ }^{99 \mathrm{~m} T c-M A A}\right)$ and $B\left({ }^{90} \mathrm{Y}\right.$ microsphere) and $p_{A, B}$ is the joint probability distribution of $A \& B$. The method which gave the highest $I(A, B)$ was used for registering the images. Details of the calculation of mutual information is explained by Maes et al. (74). Mean CPU time to register two images was about 15 minutes on a standard PC.

A)
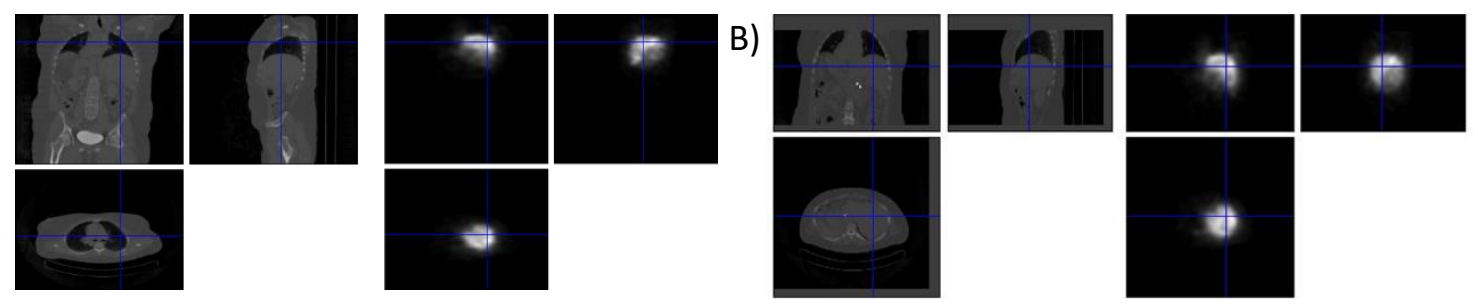

C)
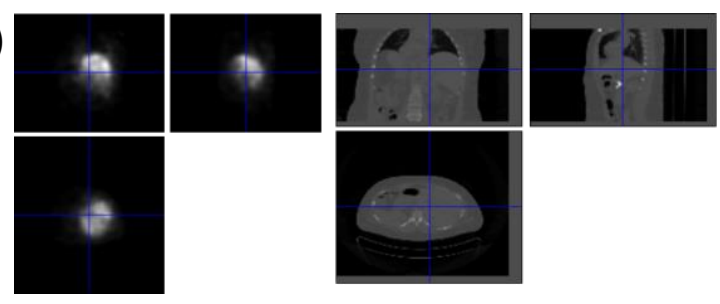

Figure 33: Registration via changing the fixed image: (A) CT of pre-treatment, (B) CT of posttreatment and (C) SPECT of post-treatment.

\subsubsection{Tumor segmentation}

Tumor segmentation was important to compare the uptake patterns in the pre and posttreatment images. Quantitative uptake analysis on ${ }^{99 \mathrm{~m} T c-M A A ~ S P E C T / C T ~ f o r ~ t u m o r ~ d e l i n e a t i o n ~ i s ~}$ usually done using an isocontour method with a dedicated software (9). Tumor segmentation based on global thresholding approach is the simplest and most popular technique in image segmentation (75). However, this method is not feasible for our project. Applying a global thresholding on two functional images with huge differences in the administered activity imposes a bias favoring either of the two images depending on the value of the threshold, as shown in Fig. 34. Thus, we sought a method that is specific to the image of interest, and also considers the noise and abrupt changes in image intensity values. The latter criteria are a requirement of our study due to the distribution of 
${ }^{99} \mathrm{Tc}-\mathrm{MAA}$ and ${ }^{90} \mathrm{Y}$ microspheres which are expected to be localized more in tumor tissue than in the healthy liver (31). Thus, the challenge here is to accurately extract tumor contours.
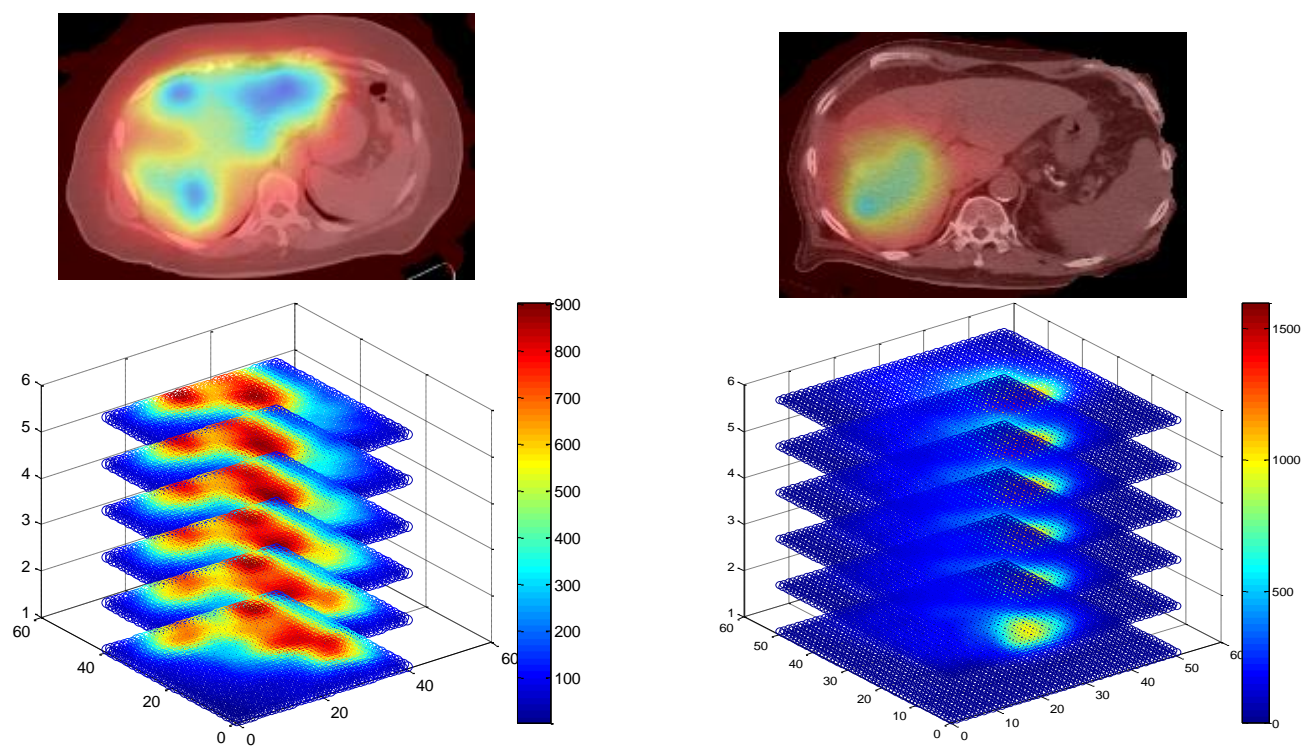

Figure 34: Scatter plot of ${ }^{90} \mathrm{Y}$ microsphere SPEC images for patient 10 (left) and 13 (right) with administered activity of 1061.90 and $1235.8 \mathrm{MBq}$ respectively.

To overcome these challenges tumor segmentation based on the uptake of ${ }^{99 \mathrm{~m}} \mathrm{Tc}-\mathrm{MAA}$ and ${ }^{90} \mathrm{Y}$ microspheres was performed using an active contour segmentation method to delineate areas of high activity (tumor) from the surrounding low activity (healthy) liver.

\subsubsection{Active contour based tumor segmentation}

Active contour detects specified features by evolving a curve in a given image where the evolution stops when the curve meets a boundary (76). The magnitude of the gradient of the image is used to stop the curve. But the gradient based methods suffer a limitation from the fact that only edges defined by the gradient of the local information are detected. An alternative is to use a global segmentation of the image to stop the curve. Chan-Vese (CV) et al. (77) has developed an active contour method that addresses this limitation by introducing an energy based segmentation via a multi-phase level set approach (Eqn. 36). The CV method is an iterative technique that allows for automatic detection of interior contours, and segments images with complex topologies into 
multiple segments (77). This technique is particularly important in tumors with a necrotic core where there is a minimal uptake within the core. The iteration number for the algorithm was chosen based on the convergence criteria where the segmented tumor volume no longer changes in size (Fig. 35).

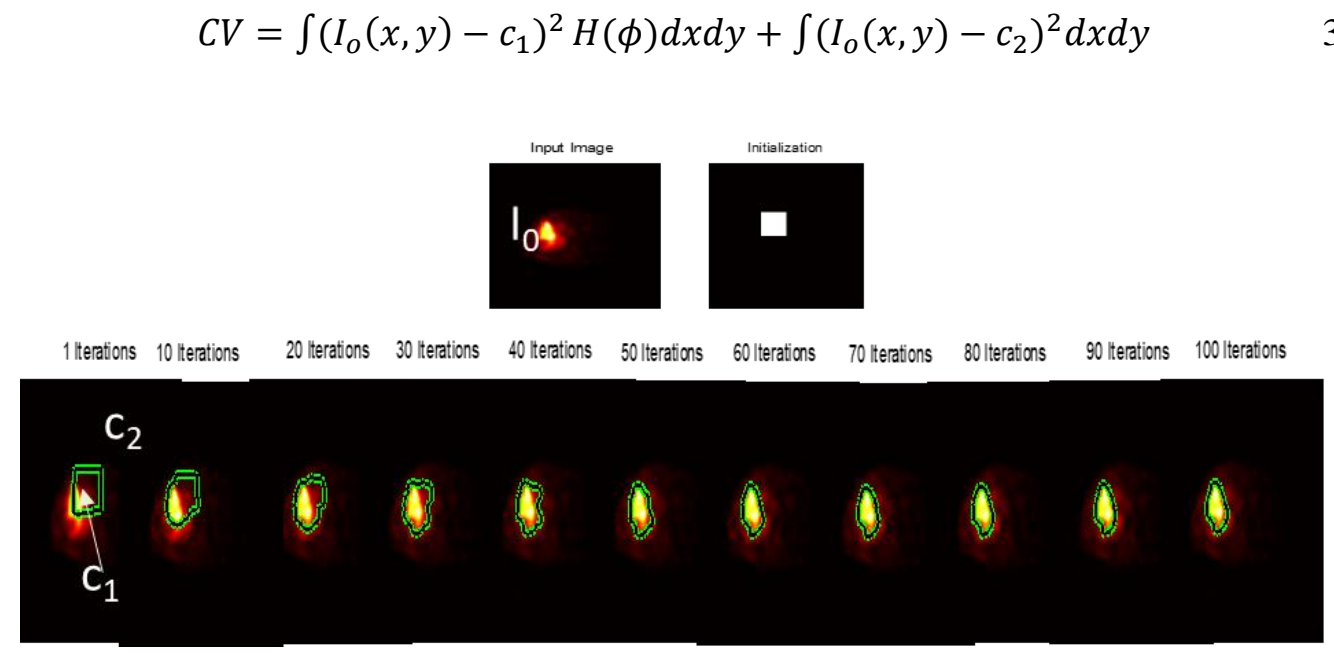

Figure 35: The iterative CV active contour segmentation method for different iteration number. $I_{o}(x, y)$ is intensity of the input image, $c_{1}$ and $c_{2}$ are mean intensities within the inside and outside contour curve respectively, and $H()$ and $\phi$ are the Heaviside and level set functions respectively.

\subsubsection{Tumor to healthy liver ratio}

In order to avoid the bias of very high and very low counts we used a mean based ratio to calculate the TLRs. As counts are proportional to activity concentration, the TLR for a given tumor was defined as,

$$
T L R=\frac{\bar{t}(i, j, k)}{\overline{b k g}(i, j, k)}
$$

$\bar{t}$ and $\overline{b k} g$ are the mean counts per pixel of the tumor and healthy liver respectively. 


\subsection{Results and Discussion}

\subsubsection{Co-registration of ${ }^{99 \mathrm{~m} T c-M A A}$ and ${ }^{90} \mathrm{Y}$ SPECT/CT}

Figure 36 shows results of the three co-registration approaches where the CT scan of the post-treatment as a reference image gave the best alignment between the images. Using the ${ }^{90} \mathrm{Y}$ microsphere SPECT image as a reference also gave a good result, visually comparable to the aforementioned result. Figure 37 shows plots of the probability distribution of the pre and posttreatment images and their joint distribution after co-registration which were used for subsequent calculation of the mutual information. The mutual information is higher or equal in most patients where the CT scan was the reference image [Fig. 38(A)]. In other patients, for example patient 11, the ${ }^{99 \mathrm{~m}} \mathrm{Tc}-\mathrm{MAA}$ and ${ }^{90} \mathrm{Y}$ microsphere SPECT images didn't co-register correctly with the CT image. From the SPECT scans of this patient, we observed that the patient had a hepatic tumor with a necrotic core with minimal uptake inside the liver, which reduced the mutual information required for co-registering the SPECT and CT scans [Fig. 38(B)]. Patient 6 showed the smallest of all mutual information between ${ }^{99 \mathrm{~m} T c-M A A}$ and ${ }^{90} \mathrm{Y}$ microsphere SPECT scans and like the previously mentioned patient, the SPECT scan didn't co-register well with the CT scan. We observed that the

${ }^{99 \mathrm{~m}} \mathrm{Tc}-\mathrm{MAA}$ and ${ }^{90} \mathrm{Y}$ microsphere SPECT scans of this patient have exclusively localized activity in the left lobe (Fig. 39). For these patients image analyses on the ${ }^{99 \mathrm{~m}} \mathrm{Tc}-\mathrm{MAA}$ and ${ }^{90} \mathrm{Y}$ microsphere SPECT/CT were performed separately. 


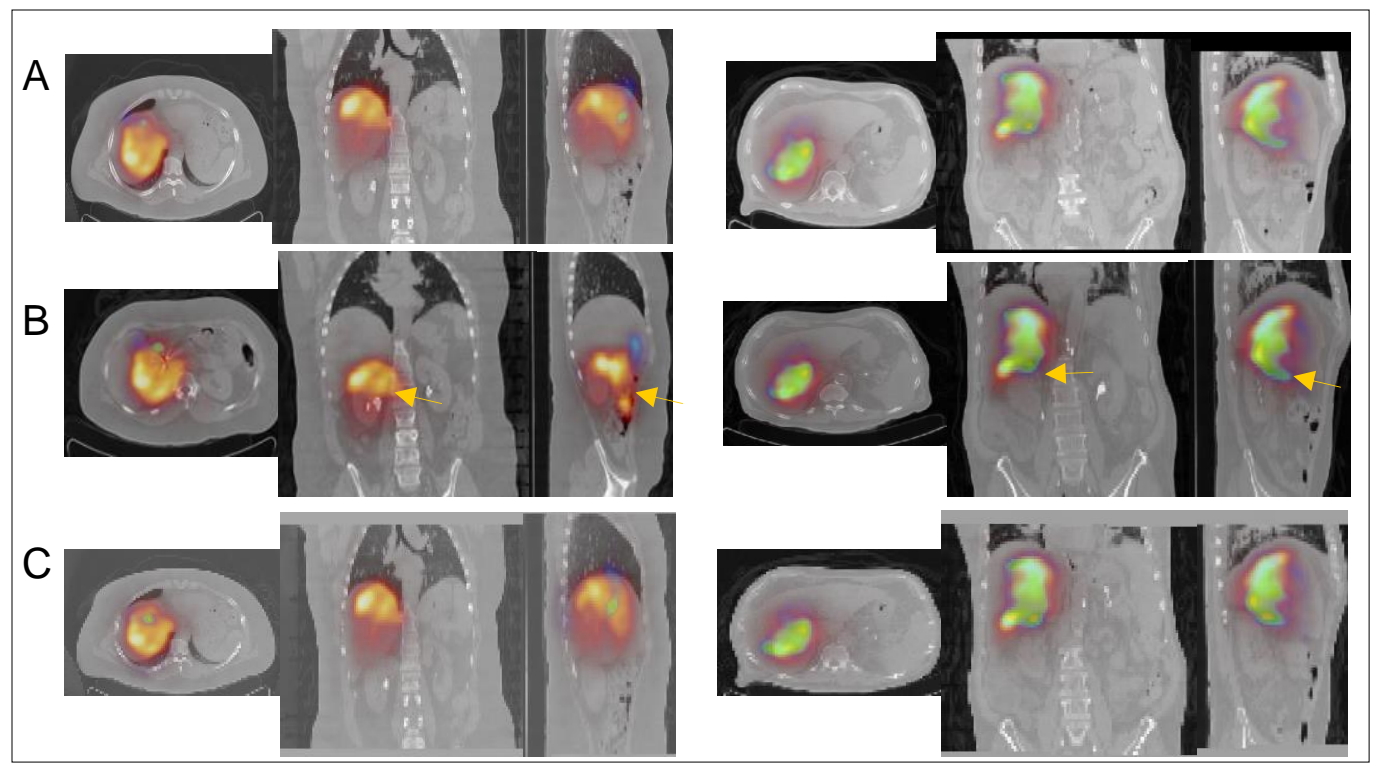

Figure 36: Patient 9 (left) and 13 (right) co-registered CT and SPECT images of ${ }^{99 \mathrm{~m} T c-M A A}$ (blue) and ${ }^{90} \mathrm{Y}$ (red) for reference images of CT of post-treatment (row A), CT of diagnostic (row B) and ${ }^{90} \mathrm{Y}$ microsphere SPECT (row C). The arrows in (B) show the misalignment between the CT and SPECT images showing activity distributions outside of the liver boundary in the coronal and sagittal views.

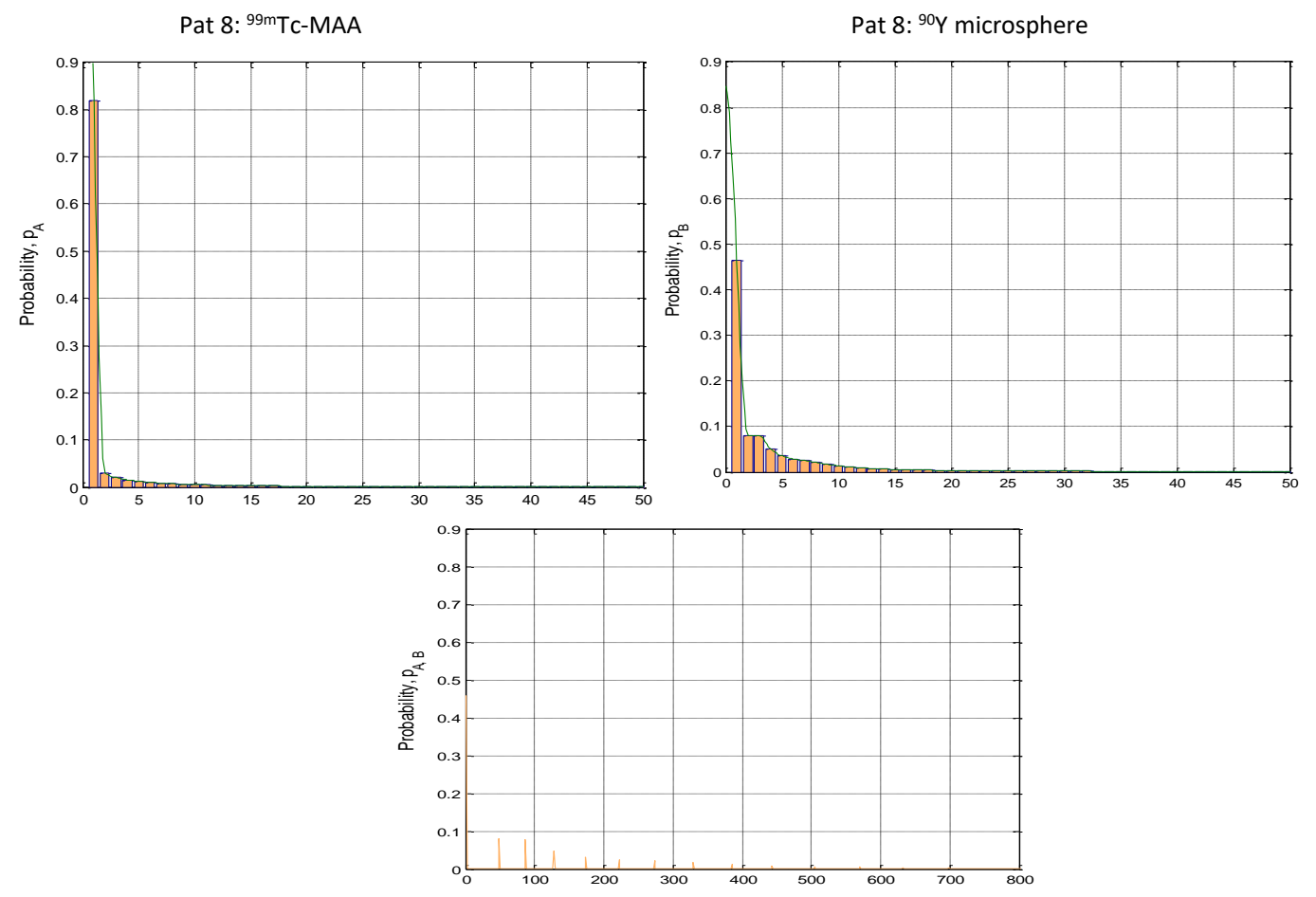

Figure 37: Plot of the probability distribution of the pre and post-treatment (upper) images and their joint distribution after co-registration. 


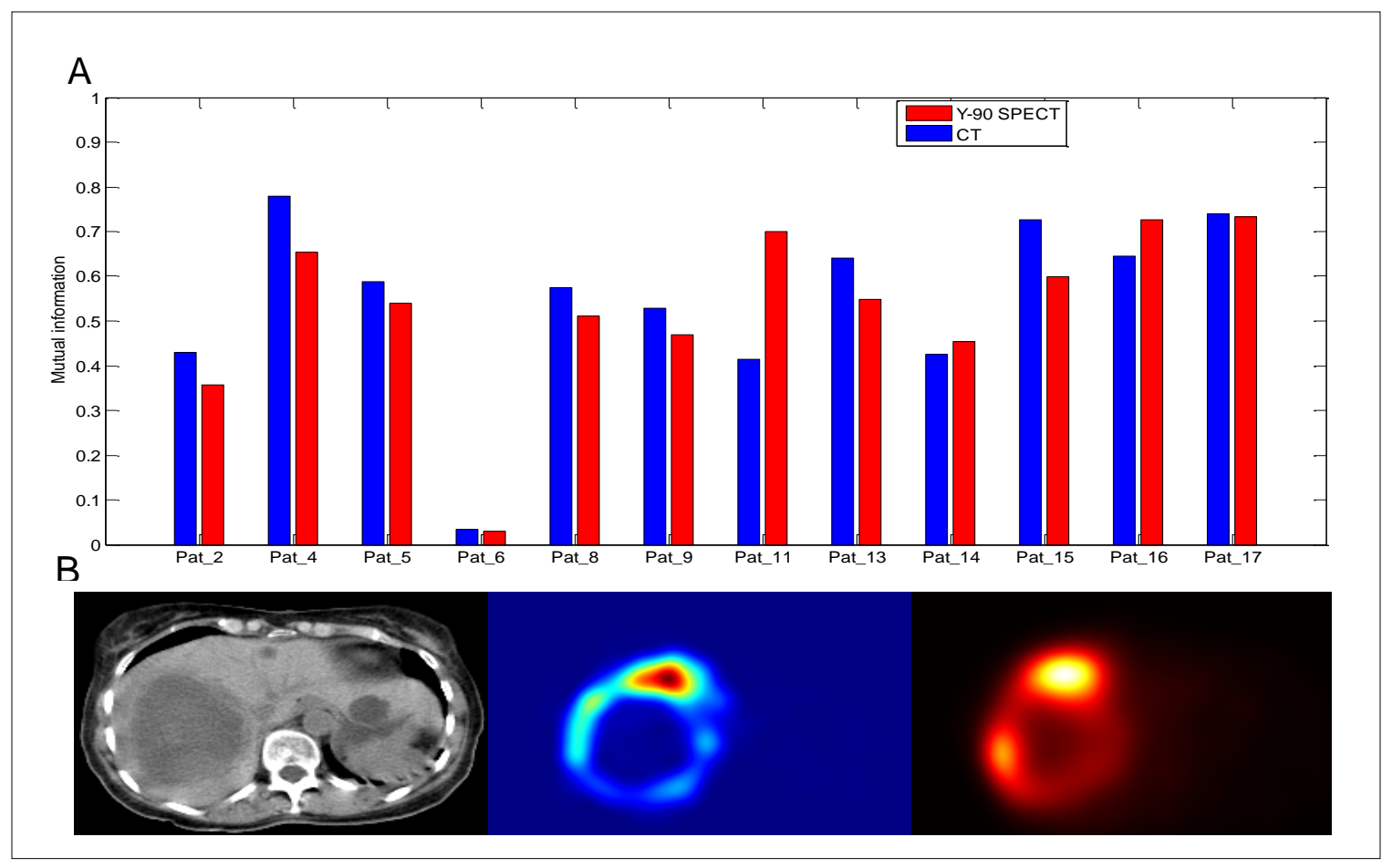

Figure 38: (A) Mutual information between co-registered ${ }^{90} \mathrm{Y}$ microsphere and ${ }^{99 \mathrm{~m}} \mathrm{Tc}-\mathrm{MAA}$ SPECT images. (B) Patient $11 \mathrm{CT}$ (left), ${ }^{99 \mathrm{~m} T c-M A A}$ (middle) and ${ }^{90} \mathrm{Y}$ microsphere (right) SPECT scans showing a minimal uptake within the liver.

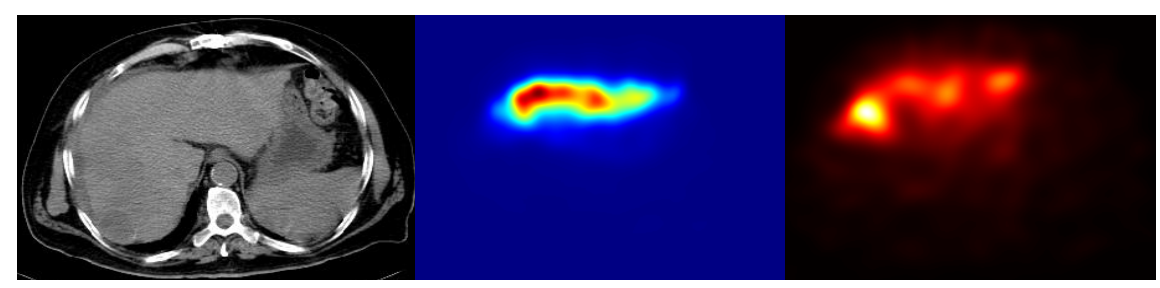

Figure 39: Patient $6 \mathrm{CT}$ (left), ${ }^{99 \mathrm{~m} T c-M A A}$ (middle) and ${ }^{90} \mathrm{Y}$ microsphere (right) SPECT that showed the smallest mutual information between the co-registered pre and post-treatment images.

\subsubsection{Tumor segmentation}

The ${ }^{99 m}$ Tc-MAA and ${ }^{90} \mathrm{Y}$ microsphere SPECT/CT images showed variable iteration number of convergence (Fig. 40). For consistency of applying similar iteration number to both images, we applied 400 iterations. Manual segmentation of whole liver for all patients ranged between 1120 - 3389 (mean: $2345 \pm 740$ ) $\mathrm{ml}$. For ${ }^{99 \mathrm{~m} T c-M A A}$, tumor volumes ranged between $160-1010(695 \pm 275) \mathrm{ml} .{ }^{90} \mathrm{Y}$-microshpere uptake gave tumor volumes in the range of $207-$ 
$1868(786 \pm 462) \mathrm{ml}$. Figure 41 shows the Box-Whisker plot of the three volumes. ${ }^{90} \mathrm{Y}$ -

microsphere SPECT/CT images gave an overall higher tumor volume for most patients compared to the ${ }^{99 m}$ Tc-MAA SPECT/CT images. A paired t-test of the two volumes gave insignificant difference $(\mathrm{t}$-value $=-0.82, \mathrm{p}$-value $=0.429)$, as shown in Table 13 .

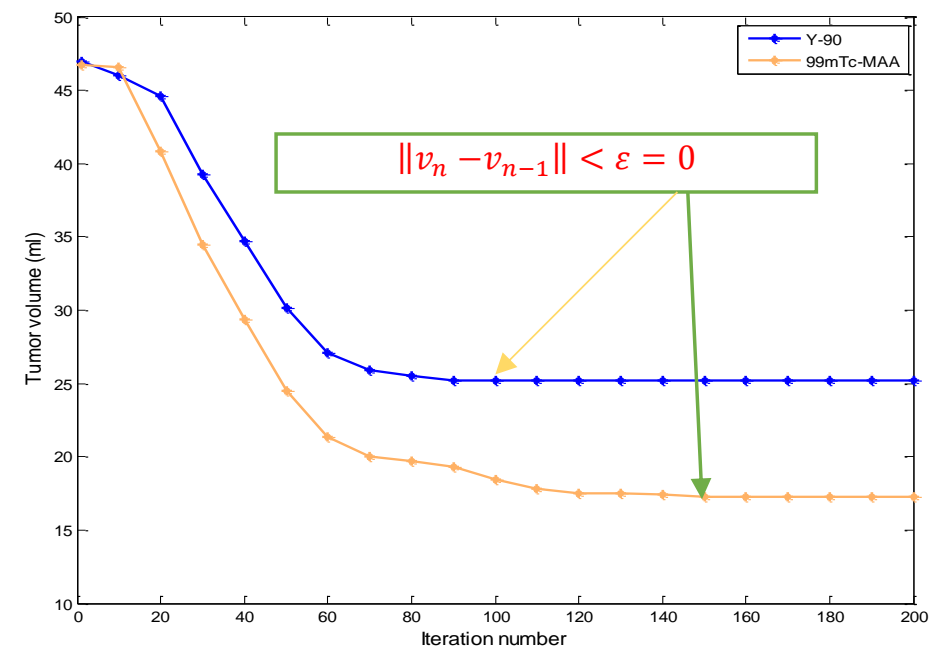

Figure 40: Plot of the segmented tumor volumes at each iteration for determining optimal iteration number $(n)$ at the convergence $(\varepsilon)$ calculated between consecutive volumes $\left(v_{n}\right.$ and $\left.v_{n-1}\right)$

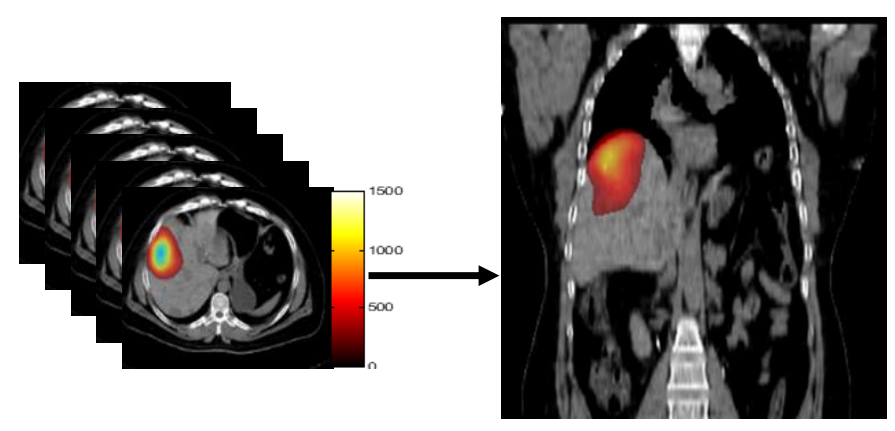

Figure 41: Segmented tumor in axial and coronal view. 


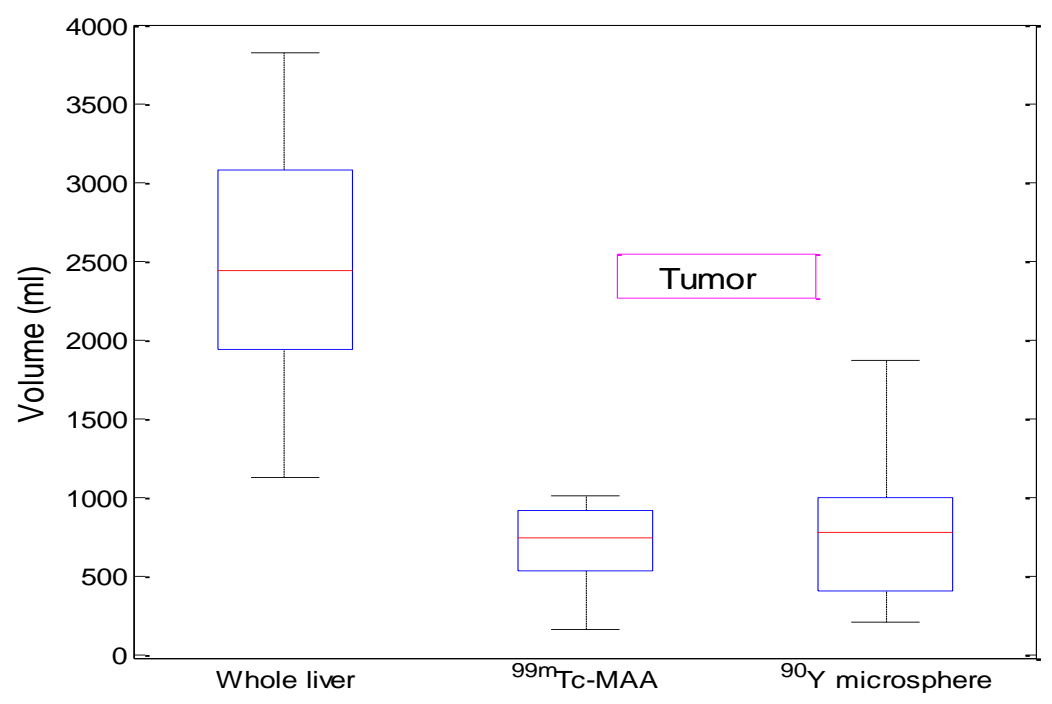

Figure 42: Box plot of whole liver and tumor segmented volumes.

Table 13: Minitab result of paired T-test between pre and post-treatment tumor volumes.

\begin{tabular}{|c|c|c|c|c|}
\hline Paired $\mathrm{T}$ for $99 \mathrm{~m}$ & $\begin{array}{c}\mathrm{C}-\mathrm{M} Z \\
\mathrm{~N}\end{array}$ & $\begin{array}{l}A-Y- \\
\text { Mean }\end{array}$ & $\begin{array}{l}0 \text { micr } \\
\text { StDev }\end{array}$ & $\begin{array}{l}\text { Dsphere } \\
\text { SE Mean }\end{array}$ \\
\hline 99mTC-MAA & 12 & 695 & 275 & 79 \\
\hline Y-90 microsphere & 12 & 786 & 462 & 133 \\
\hline Difference & 12 & -91 & 383 & 110 \\
\hline
\end{tabular}

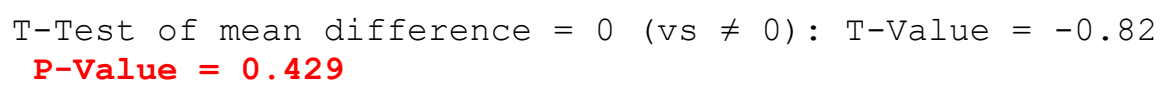

\subsubsection{TLR calculation and comparison}

Table 14 presents results of the TLRs for each patient. The total mean TLR was 9.2 \pm 9.4 and $5.0 \pm 2.2$ on ${ }^{99 \mathrm{~m} T c-M A A}$ and ${ }^{90} \mathrm{Y}$ microsphere SPECT/CT respectively. Figure 43 shows the scatterplot of mean TLRs from the two images displaying a significant correlation $(r=0.9, p<0.05)$. From the plot, patient 2 is an outlier (Grubbs' outlier test, p-value $=0.00$ ) and taking this patient out of the analysis gave a reduced correlation $(\mathrm{r}=0.6, \mathrm{p}<0.05)$. 
Table 14: Mean TLR on ${ }^{99 \mathrm{~m} T c-M A A}$ and ${ }^{90} \mathrm{Y}$ SPECT images.

Tumor to liver ratio (TLR)

\begin{tabular}{|c|c|c|}
\hline Patient no. & ${ }^{99} \mathrm{~m}$ Tc-MAA & ${ }^{90} \mathrm{Y}$ \\
\hline Pat_2 & 36.3 & 11.5 \\
\hline Pat_4 & 7.0 & 3.5 \\
\hline Pat_5 & 4.8 & 4.8 \\
\hline Pat_6 & 11.7 & 5.0 \\
\hline Pat_8 & 5.6 & 4.3 \\
\hline Pat_9 & 2.7 & 2.6 \\
\hline Pat_11 & 3.8 & 4.2 \\
\hline Pat_13 & 10.2 & 5.3 \\
\hline Pat_14 & 4.4 & 4.8 \\
\hline Pat_15 & 1.8 & 3.8 \\
\hline Pat_16 & 7.5 & 4.6 \\
\hline Pat_17 & 14.9 & 5.0 \\
\hline Mean & 9.2 & 5.0 \\
\hline Std. & 9.4 & 2.2 \\
\hline
\end{tabular}




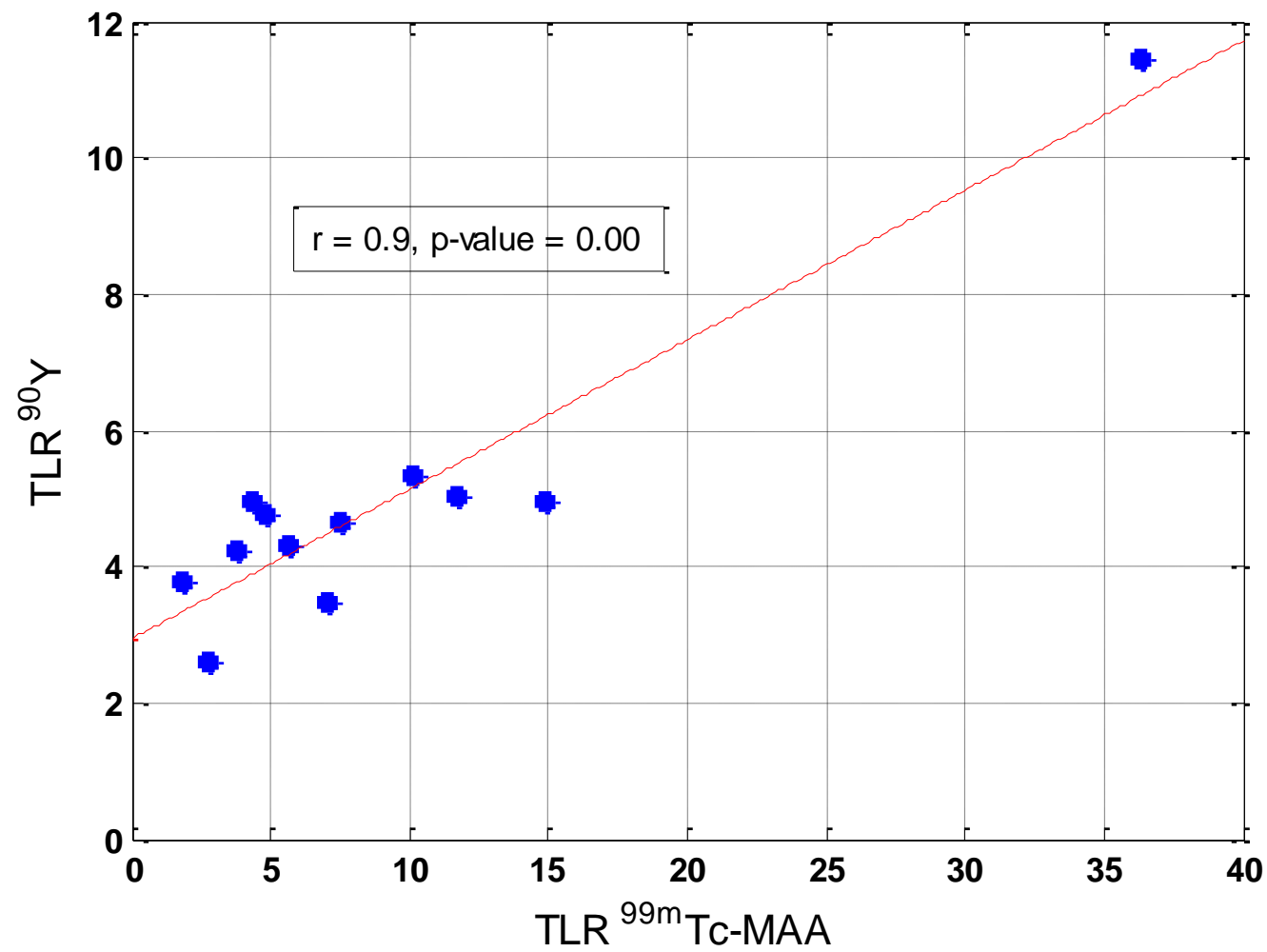

Figure 43: Scatterplot illustrating correlation between mean TLRs in 90Y and 99mTc-MAA SPECT.

Comparing the ${ }^{99 \mathrm{~m}} \mathrm{Tc}-\mathrm{MAA}$ distribution of patient 2 with other similar patient scans (Fig. 44) revealed that the localized distribution might have contributed to the higher TRL ratio as there is minimal or no significant uptake in the healthy liver. But this scenario needs further investigation, which can be achieved by dividing the patient cohort into two, those who had left or right lobe treatment. This kind of comparison will help decide if the TLR ratio is affected by a localized vs. a decentralized distribution for the specific lobe treatment. 

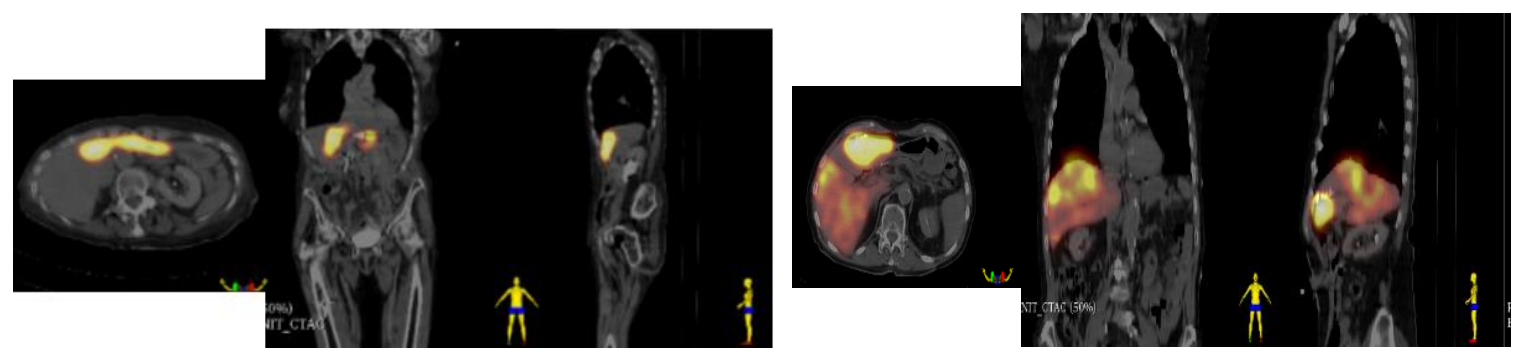

Figure 44: Comparison of ${ }^{99 \mathrm{~m}} \mathrm{Tc}-\mathrm{MAA}$ SPECT/CT uptake distribution between patient 2 (left) and patient 8 (right).

The predictive accuracy of ${ }^{99 \mathrm{~m}} \mathrm{Tc}-\mathrm{MAA}$ regarding the actual ${ }^{90} \mathrm{Y}$ microspheres dose distribution and its impact on patient outcomes is a source of controversy. Ilhan et al. (10) have studied the relationship between ${ }^{99 \mathrm{~m}} \mathrm{Tc}-\mathrm{MAA}$ and ${ }^{90} \mathrm{Y}$ microspheres uptake in a retrospective study which involved 502 patients with various liver cancer types (10). This study found a weak correlation between the mean tumor to background (healthy liver) ratios of ${ }^{99 \mathrm{~m}} \mathrm{Tc}-\mathrm{MAA}$ SPECT/CT and ${ }^{90} \mathrm{Y}$ microsphere SPECT/CT images. We believe a comparison of the pre and post-treatment images of ${ }^{90} \mathrm{Y}$ microspheres and ${ }^{99 \mathrm{~m}} \mathrm{Tc}-\mathrm{MAA}$ may help evaluate the discordance between MAA distributions versus actual microsphere distribution. There are known factors that could contribute to discrepancies. First and foremost is the variable size and shape of the macro-aggregated albumin particles and clusters. Additional factors may include the embolizing effect in ${ }^{90} \mathrm{Y}$, and differences in positioning of the catheter tip between the ${ }^{99 \mathrm{~m}} \mathrm{Tc}-\mathrm{MAA}$ and ${ }^{90} \mathrm{Y}$ microsphere procedures.

Prior to quantitative comparison, the pre and post-treatment SPECT/CT images were coregistered. The accuracy of the comparison strongly depends on the proper matching of the two images in all three dimensions. From our results, we found that this alignment depends on the overall content of liver activity within the SPECT scans which could be attributed to the fact that SPM is based on identifying regionally specific effects in the imaging data (2). As a result, the registration didn't provide very accurate alignment between the CT and SPECT images in cases such as a necrotic tumor covering a larger portion of the liver. In these cases, manual matching of the ${ }^{99 m} \mathrm{Tc}-\mathrm{MAA}$ SPECT/CT and ${ }^{90} \mathrm{Y}$ microsphere SPECT/CT images was necessary. 
Tumor segmentation gave higher mean volumes in the post-treatment images compared to the respective pre-treatment images. Although the difference between the volumes is insignificant (p-value $=0.429)$, since the tumor segmentation is based on higher activity concentrations, it is possible that the tumor ROIs didn't necessarily coincide with the tumorous tissues in the ${ }^{90} \mathrm{Y}$ microsphere SPECT/CT images. However, it is expected in radioembolization that higher microsphere accumulation is to be in tumorous regions rather than the healthy liver parenchyma (17).

To minimize outlier effects, we used the ratio of the mean activities between the tumorous and healthy liver to calculate the TLRs. The mean TLRs between ${ }^{99 \mathrm{~m} T c-M A A}$ and ${ }^{90} \mathrm{Y}$ microsphere SPECT/CT images showed a strong correlation with one patient as an outlier where removing this patient gave a much lower correlation. Although statistically correct, for the patient to be considered as an outlier in terms of invalid treatment the statement falls short. Ilhan et al. (10) states that the TLRs from ${ }^{99 m}$ Tc-MAA SPECT/CT is higher in all patients compared to the respective ${ }^{90} \mathrm{Y}$ microsphere SPECT/CT scans, which the authors believe to be due to the poor image quality of ${ }^{90} \mathrm{Y}$ bremsstrahlung SPECT/CT. In our study, we found that after ${ }^{90} \mathrm{Y}$ bremsstrahlung SPECT/CT image improvement the TRLs were higher in ${ }^{99 \mathrm{~m} T c-M A A ~ S P E C T / C T ~ f o r ~ m o s t ~ o f ~ t h e ~}$ patients, and others showed a higher ${ }^{90} \mathrm{Y}$ microspheres uptake over the ${ }^{99 \mathrm{~m}} \mathrm{Tc}-\mathrm{MAA}$. The standard deviation of the TLRs on ${ }^{90} \mathrm{Y}$ microspheres SPECT/CT images is lower than ${ }^{99 \mathrm{~m} T c-M A A}$ SPECT/CT which could be associated with the embolizing effect of ${ }^{90} \mathrm{Y}$ microsphere particles $(9,10)$.

\subsection{Conclusion}

The objective of this study was the comparison of the uptake distributions between pretreatment ${ }^{99 \mathrm{~m}} \mathrm{Tc}-\mathrm{MAA}$ and post-treatment ${ }^{90} \mathrm{Y}$ microsphere SPECT/CT images. The study investigated various methods and their feasibility for co-registration of the ${ }^{99 \mathrm{~m}} \mathrm{Tc}-\mathrm{MAA}$ and ${ }^{90} \mathrm{Y}$ 
microsphere SPECT/CT images. In addition, tumor segmentation suitable for pre and post RMT images was sought. In the end, we identified a substantial correlation in mean TLRs between ${ }^{99 \mathrm{~m}} \mathrm{Tc}-$ MAA and ${ }^{90} Y$ microspheres SPECT/CT uptake distribution.

\section{Chapter 7: Dosimetry algorithm}

\subsection{Introduction}

Absorbed dose (dosimetry) is the mean energy imparted to target tissue per unit mass (Eqn. 38) (78). Techniques for post-treatment ${ }^{90} \mathrm{Y}$ microsphere imaging dosimetry have grown significantly in recent years. The compartmental (partition) model by the Medical Internal Radiation Dose (MIRD) is the first method to characterize dose independently to target tumor and healthy part of the liver (79). The primary MIRD formula (non-compartmental model) used to determine absorbed dose from an imaging scan is given by Eqn. 38 where the anatomical volumes are determined based on standard human size (Fig. 45). The compartmental model however partitions the liver into the normal liver and tumor (80) given by Eqn. 39-41.

$$
\begin{gathered}
D(G y)=\frac{\text { Energy Absorbed from Ionizing Energy }(J)}{\text { Mass of Organ }(K g)} \\
D(G y)=\frac{A_{\text {inj }}(G B q) *(1-L S F) * 50}{W(k g)} \\
D(G y)_{\text {Normal }}=A_{\text {inj }}(G B q) * \frac{(1-L S F) * 50}{m_{\text {normal }}+T L R * m_{\text {tumor }}} \\
D(G y)_{\text {Tumor }}=T L R * D(G y)_{\text {Normal }}
\end{gathered}
$$

Here, $A_{i n j}$ is the injected activity, $L S F$ is the lung shunt fraction and $W$ is the weight of the vascularized hepatic volume, $T L R$ is the tumor to liver ratio, $m_{\text {normal }}$ is the mass of the normal liver 
compartment, $m_{\text {tumor }}$ is the mass of the tumor compartment, $D_{\text {Normal }}$ is the radiation absorbed dose by the normal/healthy liver and $D_{\text {Tumor }}$ is the absorbed dose by tumorous liver tissues.

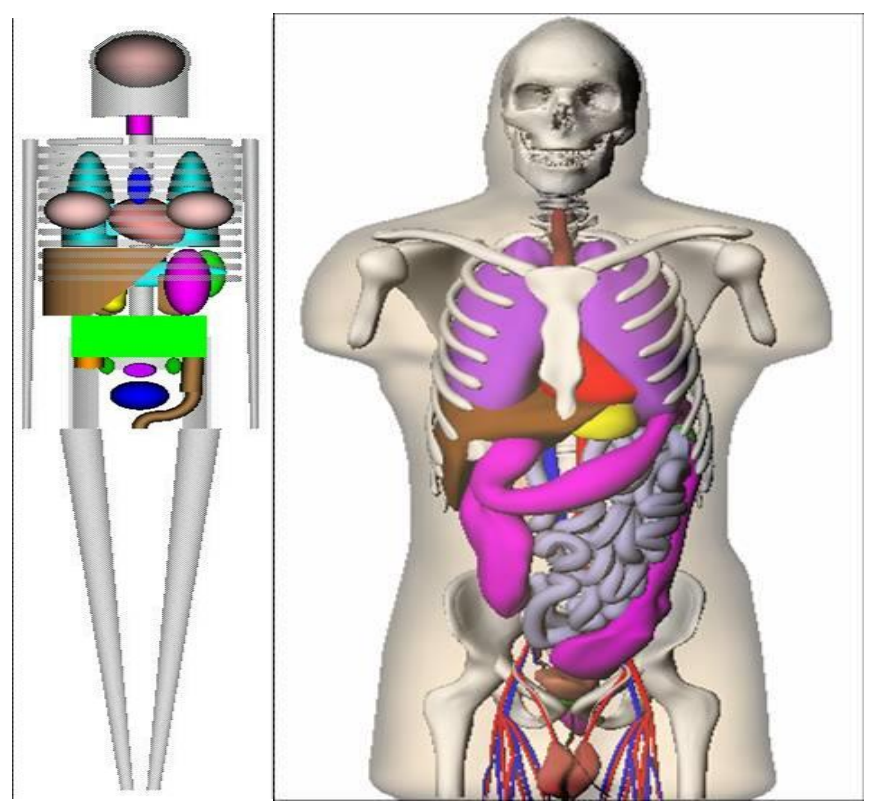

Figure 45: Human body models of older (left) and newer version (right) for standard organ sizes $(81)$.

The dose reported by these methods is, however, the mean absorbed dose in normal or tumor volumes based on the assumption of a uniform activity distribution within the source volumes (32). In RMT treatment however, the microspheres are deposited in the liver as a number of discrete clusters rather than as point sources that are homogeneously distributed throughout the tumor or healthy liver. This irregular clustering of microspheres produces a highly heterogeneous radiation dose distribution pattern. As a result errors in the order of $30-100 \%$ have been reported from the assumption of uniform distribution (30). Thus, non-uniform dosimetry methods that represent actual ${ }^{90} \mathrm{Y}$ microsphere distribution in SPECT/CT or PET/CT is essential as the mean absorbed dose to an organ calculated by the MIRD formula doesn't provide enough information to predict the potential biological effects as well as treatment efficacy. Regional or voxel-based dosimetry that addresses the nonuniform ${ }^{90} \mathrm{Y}$ microsphere distribution is thus required. SPECTbased dosimetry is more acceptable than PET since most therapeutic radionuclides are not positron 
emitters, but rather single photon emitters suitable for SPECT imaging. As previously explained in chapter 2 studies have shown the feasibility of ${ }^{90} \mathrm{Y}$ PET/CT imaging dosimetry estimation.

Voxel based dosimetry is the calculation of radiation absorbed dose to tissue regions with dimensions ranging from a few centimeters to hundreds of microns (78). Voxel based dosimetry has various approaches. One of the simplest approaches is the assumption that the emitted energy is completely absorbed locally, at the voxel where it is emitted (82). Other approaches that don't require such assumption are the voxel S value method (Fig. 46), the dose point kernel and the Monte Carlo radiation transport methods (78).

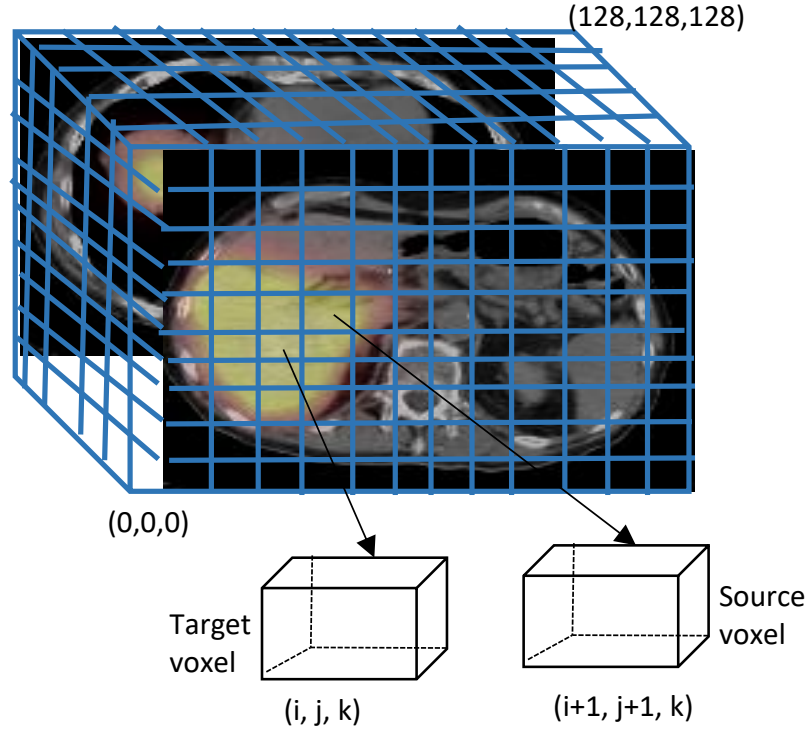

Figure 46: Illustration of voxel dosimetry

The Voxel S value method and dose point kernel are considered to be better choices than the simplified method mentioned that assumes complete local absorption and as well as the computer intensive and time consuming method based on Monte Carlo radiation transport (32). S value is 'the mean absorbed dose to a target organ per radioactive decay in a source organ, mGy/MBq s' (83). The product of source organ cumulative activity and the corresponding S value 
gives the target organ dose. MIRD S values calculated from Monte Carlo simulation are widely used for dose calculation (82). The disadvantages to using these MIRD S values are the assumption of uniform activity distribution in source organs and uniform deposition in target organ, use of anatomic models for organ sizes and shapes (Fig 44), and lack of S values for tumor volumes $(82,83)$. To compensate for these limitations of the MIRD formalism, Franquiz et al. and Bolch et al. $(78,83)$ have developed a method that calculates beta $\mathrm{S}$ values between the source and target voxel centroids that will help determine dose distribution at the maximal experimental resolution. This method helps to determine $\mathrm{S}$ values for a set of all possible combinations of cubical and noncubical pixel edges used in SPECT or PET studies.

\subsection{Materials and Methods}

The voxel S-value method was used to estimate 3D radiation absorbed dose in ${ }^{90} \mathrm{Y}$ SPECT/CT and PET/CT images. Cubical voxel $\mathrm{S}$ values for the pixel size of $4.664 \mathrm{~mm}$ were determined using linear interpolation from 3 and $6 \mathrm{~mm}$ pixel sizes estimated in Franquiz et al. and Bolch et al. (83) using Eqn. 42;

$$
\begin{gathered}
S\left(\frac{m G y}{M B q S}\right)=S_{1}+\left(V-V_{1}\right)\left(\frac{S_{2}-S_{1}}{V_{2}-V_{1}}\right) \\
S\left(\frac{m G y}{M B q}\right)=S\left(\frac{m G y}{M B q S}\right) \times T_{e f f_{-} \text {halflife }}(S) \\
\frac{1}{T_{\text {eff_halflife }}}=\frac{1}{T_{\text {bio_halflife }}}+\frac{1}{T_{\text {phy_halflife }}}
\end{gathered}
$$

$S_{1}, S$ and $S_{2}$ are the $S$ values and $V_{1}, V$ and $V_{2}$ are the volumes for the 3, 4.66 and $6 \mathrm{~mm}$ pixel sizes respectively, $T_{\text {eff_halfilife }}$ if the effective half-life calculated from the biological half-life, $T_{\text {bio_halfife }}$ and physical half-life, $T_{\text {phy_halfife, }}$, which are 49 years and 2.67 days respectively for ${ }^{90} \mathrm{Y}$. A similar method was followed for calculating the $\mathrm{S}$ values for ${ }^{99 \mathrm{~m}} \mathrm{Tc}$ SPECT/CT images. Figure 47 shows that fractions of absorbed dose for electrons (beta particles) starts out big but there is a rapid decrease in dose fraction after the electron range, which is $11 \mathrm{~mm}$ for ${ }^{90} \mathrm{Y}$. After this point the fractional dose in the range of $10^{-7}$ and $10^{-8}$ is mainly due to the bremsstrahlung photons. For 
${ }^{99 \mathrm{~m}} \mathrm{Tc}$, which is a gamma emitter, although higher dose fraction is seen within the $11 \mathrm{~mm}$ range there is a substantial dose contribution well beyond this range demanding the inclusion of far more number of surrounding voxels in dose calculations. S values were generated for the positive (first) octant only due to the symmetry of values in the negative octants.
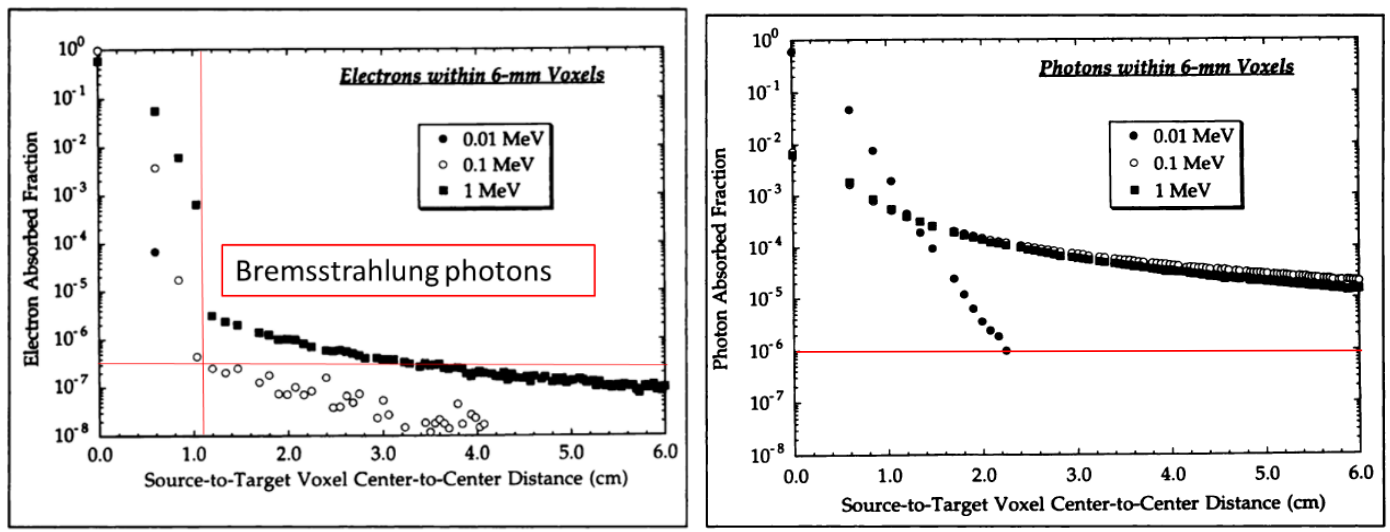

Figure 47: Plots of absorbed dose fraction for electrons (left) and photons of a $6 \mathrm{~mm}$ voxel on edge (78).

Absorbed dose per voxel was estimated by convolution of the cumulative activity images with the corresponding voxel S values, implemented using a MATLAB ${ }^{\circledR}$ algorithm (Eqn. 45) based on the MIRD Pamphlet No. 17 (78).

$$
D_{T}=\tilde{\mathrm{A}} \otimes S=\tilde{\mathrm{A}}_{S 1} \times S_{T \leftarrow S 1}+\tilde{\mathrm{A}}_{S 2} \times S_{T \leftarrow S 2}+\tilde{\mathrm{A}}_{S 3} \times S_{T \leftarrow S 3} \cdots
$$

$D_{T}$ is the absorbed dose at the target voxel (mGy), $\tilde{A}(\mathrm{MBq})$ is the time-integrated cumulative activity in a voxel from sequential imaging, $\otimes$ is the $3 \mathrm{D}$ convolution and $S$ is the voxel $\mathrm{S}$-value $(\mathrm{mGy} / \mathrm{MBq})$ for each associated distance from the target voxel. For the ${ }^{90} \mathrm{Y}$-microshpere dosimetry calculation, cumulative activity isn't used due to the permanent implant of the microsphere where there is no elimination, redistribution and washout phases of the radiopharmaceutical. Thus a single imaging performed after microsphere administration is used for dosimetry estimation where sequential imaging after day 1 isn't usually performed (84). Thus in Eq. (45) activity from a single 
scan, $A$, is used instead of $\tilde{A}$ at each voxel. In addition to our method, we estimated dosimetry using the MIRD partition model (Eq.39-40) and using S values generated by a direct MC method for a 4.8mm edge voxel (referred to here as MC method for ease of understanding) as reported elsewhere (85).

Dose-volume histogram (DVH) is a display of 3D dose distribution in relation to the target volume and normal structure [Fig. 48(A)]. It is a direct and informative method of assessing a treatment plan via representing the frequency distribution (histogram) of doses over a given volume (target) for each voxel in 3D. DVH provides easily interpretable 2D graphs from the vast 3D radiation dose information. There are two types of DVH: Differential DVH (dDVH) where the percentage or absolute volume receiving dose in a corresponding dose bin is calculated, and cumulative DVH (cDVH) where the percentage or absolute volume receiving greater than or equal to the value in a corresponding dose bin is calculated. Generation of DVH is only possible for a voxel based dosimetry thus the MIRD method can't provide the details of dosimetry estimation [Fig. 48(B)]. cDVHs and Isodose curves (Eqn. 46) were generated for the tumor and healthy liver VOIs and fused with the SPECT/CT dose map images using a third-party software for a 3dimensional display called 3DSLICER, an open source software for medical image processing and $3 \mathrm{D}$ visualization $(86)$.

$$
\text { isodose }(x, y, z)=\frac{\operatorname{Dose}(x, y, z)}{\operatorname{Max} \_ \text {Dose }(x, y, z)} \times 100 \%
$$




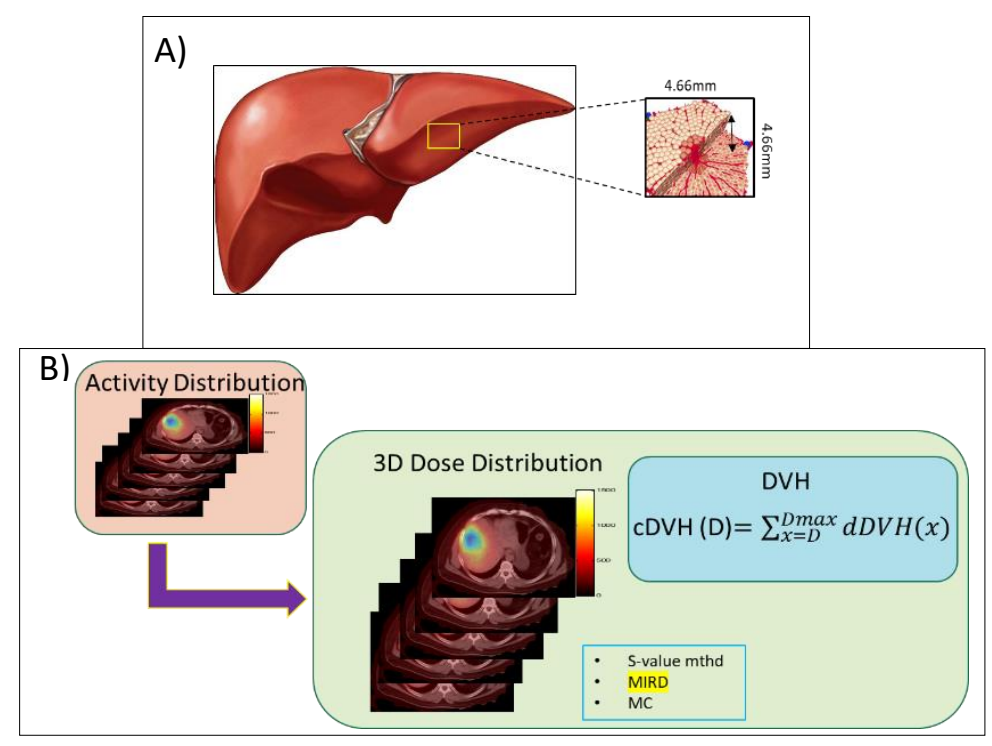

Figure 48: Display of details provided by DVH (A) and its implementation from activity and dose map images (B) where this implementation isn't possible for the MIRD method highlighted.

\subsection{Results and Discussion}

Generated voxel S-values are shown in Fig. 49 for ${ }^{90}$ Y. Figure 49 shows plots of voxel $S$ values for different distances within the beta range for ${ }^{90} \mathrm{Y}$ and ${ }^{99 \mathrm{~m}} \mathrm{Tc}$. The plots resemble Fig. 47 of values generated by direct MC simulation. The mean absorbed doses for the tumor and healthy liver from ${ }^{90} \mathrm{Y}$ microsphere SPECT/CT images were $62.6 \pm 20.2$ (range: 38.4 to $117.2 \mathrm{~Gy}$ ) and 12.4 \pm 4.7 (range: 6 to $23.7 \mathrm{~Gy}$ ) respectively using our method.

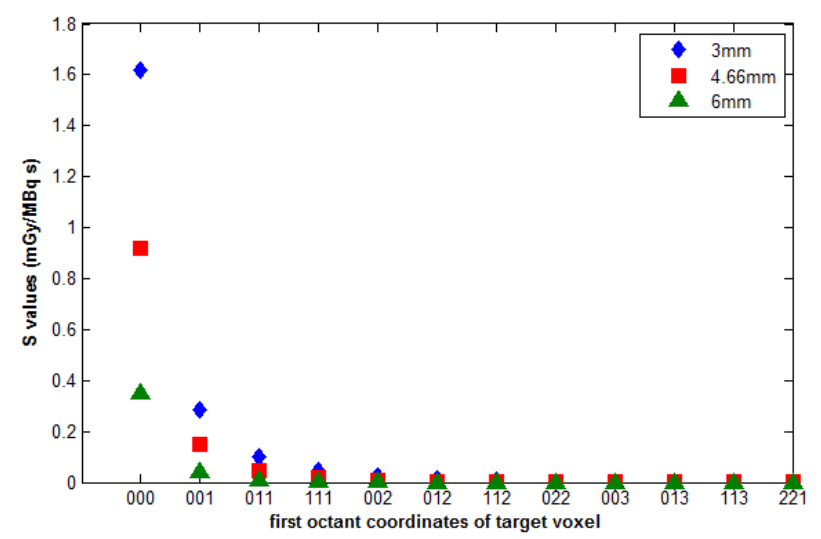

Figure 49: $\mathrm{S}$ values for various voxel sizes and interpolated results for $4.664 \mathrm{~mm}$ voxel size at different source coordinates. 

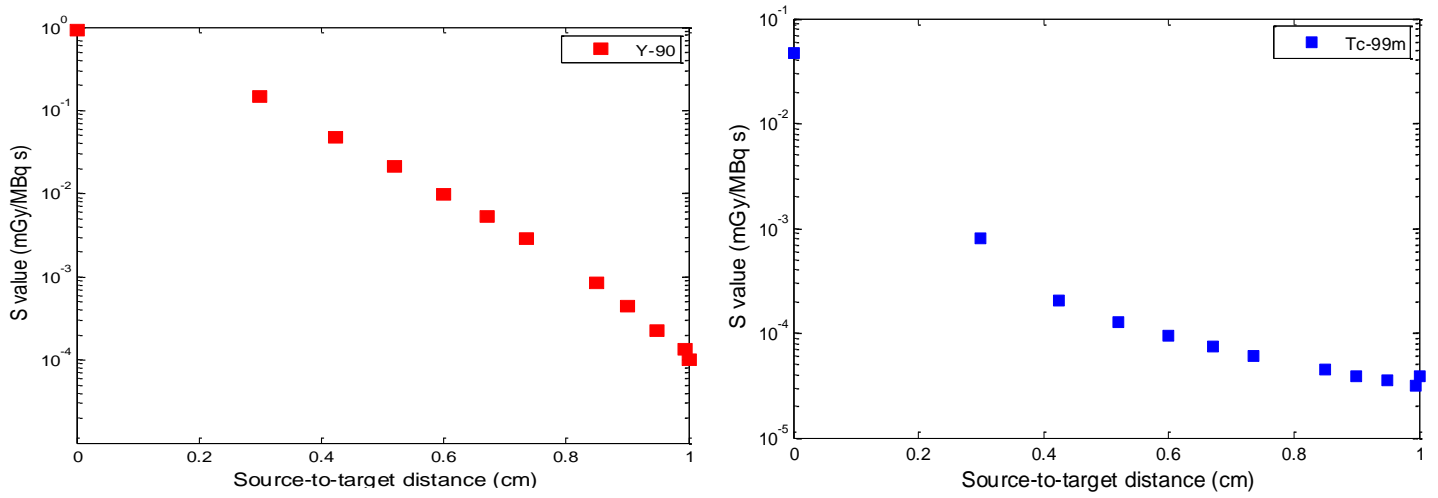

Figure 50: Calculated voxel S values for ${ }^{90} \mathrm{Y}$ (left) and ${ }^{99 \mathrm{~m}} \mathrm{Tc}$ (right) within the beta range.

The mean absorbed doses in tumor and liver volumes calculated by the three methods: proposed (our method), MC (voxel size $=4.8 \mathrm{~mm}$ ) and MIRD partition model are shown in Fig. 51 . For the MIRD method mass of tumor and liver were calculated using the segmented volumes and density of a soft tissue $\left(1.03 \mathrm{~g} / \mathrm{cm}^{3}\right)$; mass $(\mathrm{g})=$ density $\left(\mathrm{g} / \mathrm{cm}^{3}\right) \times$ volume $\left(\mathrm{cm}^{3}\right)$. Figure 52 summarizes the distribution of calculated absorbed doses in the tumor and liver volumes by the three methods. Overall higher doses are found by our method while the MC method gave the smallest estimated doses in both volumes.

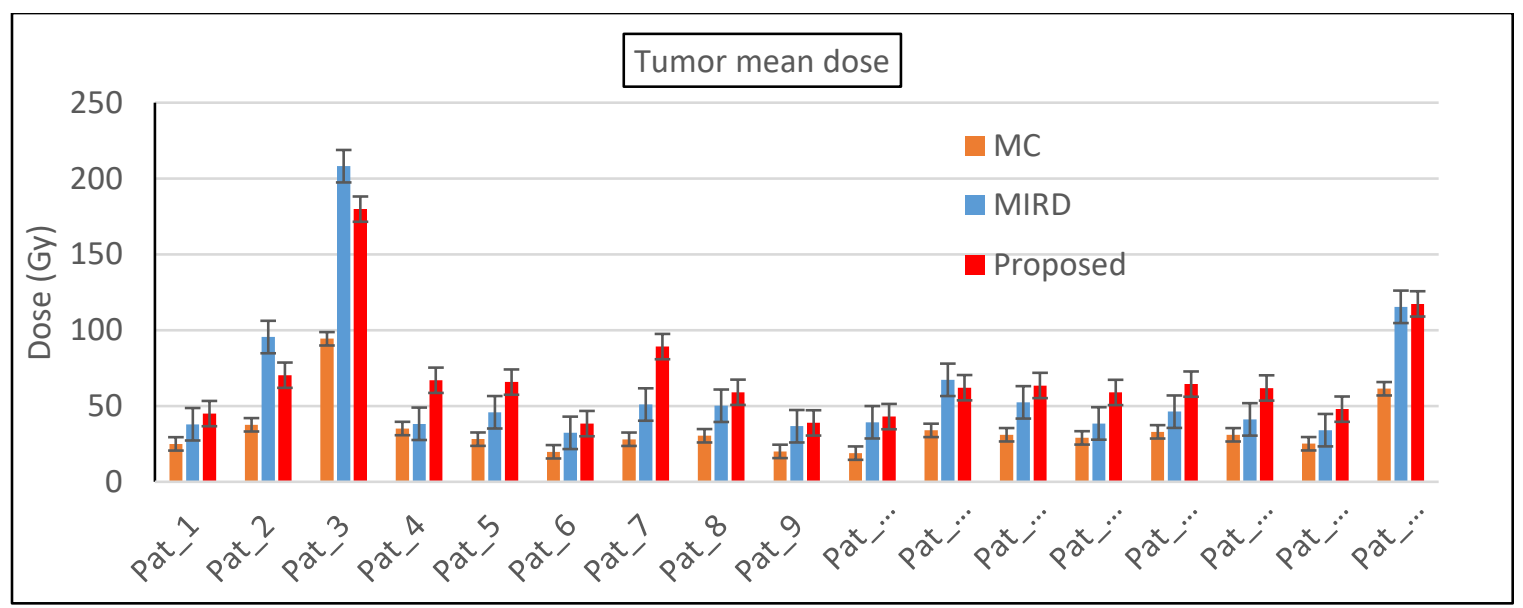




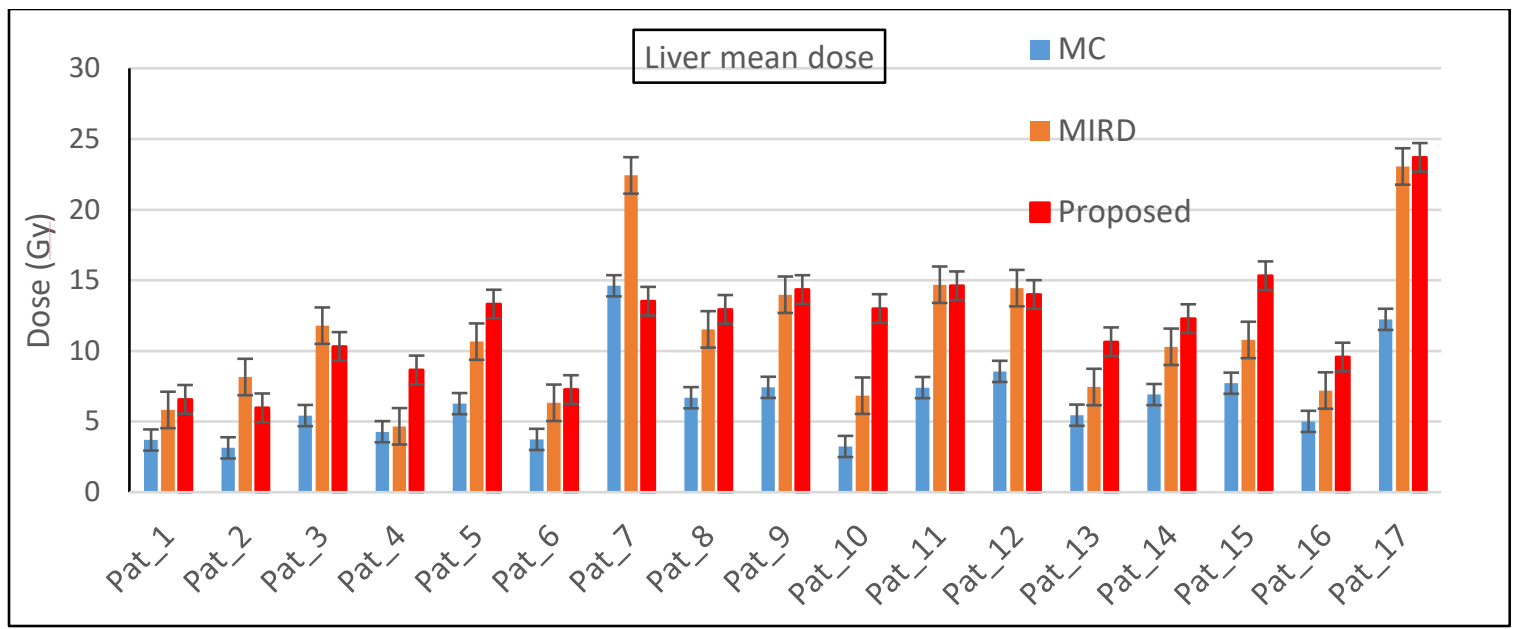

Figure 51: Bar plots of tumor (upper) and liver (lower) mean absorbed doses calculated by the three methods.
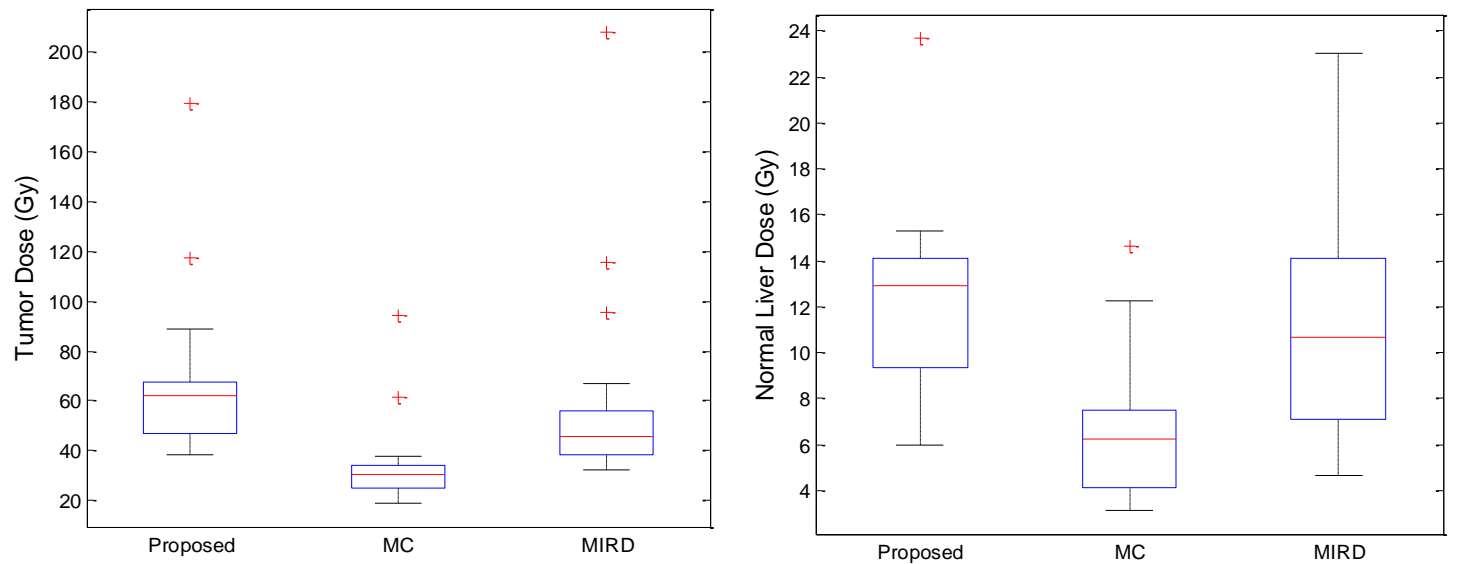

Figure 52: Box-Whisker plots of the calculated dose distribution in the tumor (left) and liver (volumes).

Figure 53 shows the $\mathrm{cDVH}$ for all patients in the tumor and liver VOIs. The tumor cDVH showed similar dose absorption scheme for all patients [Fig 53(A)] except for patient 3 who showed the highest dose per tissue volume. The isodose curves fused on the patient CT scan showed a much localized treatment dosimetry for a relatively low administered dose (558.7 MBq) with maximum absorbed dose of 500 Gy [Fig. 53(B)]. The cDVH for the liver VOIs showed that absorbed doses are within the maximum tolerated dose of 40 Gy by a liver tissue (87). 

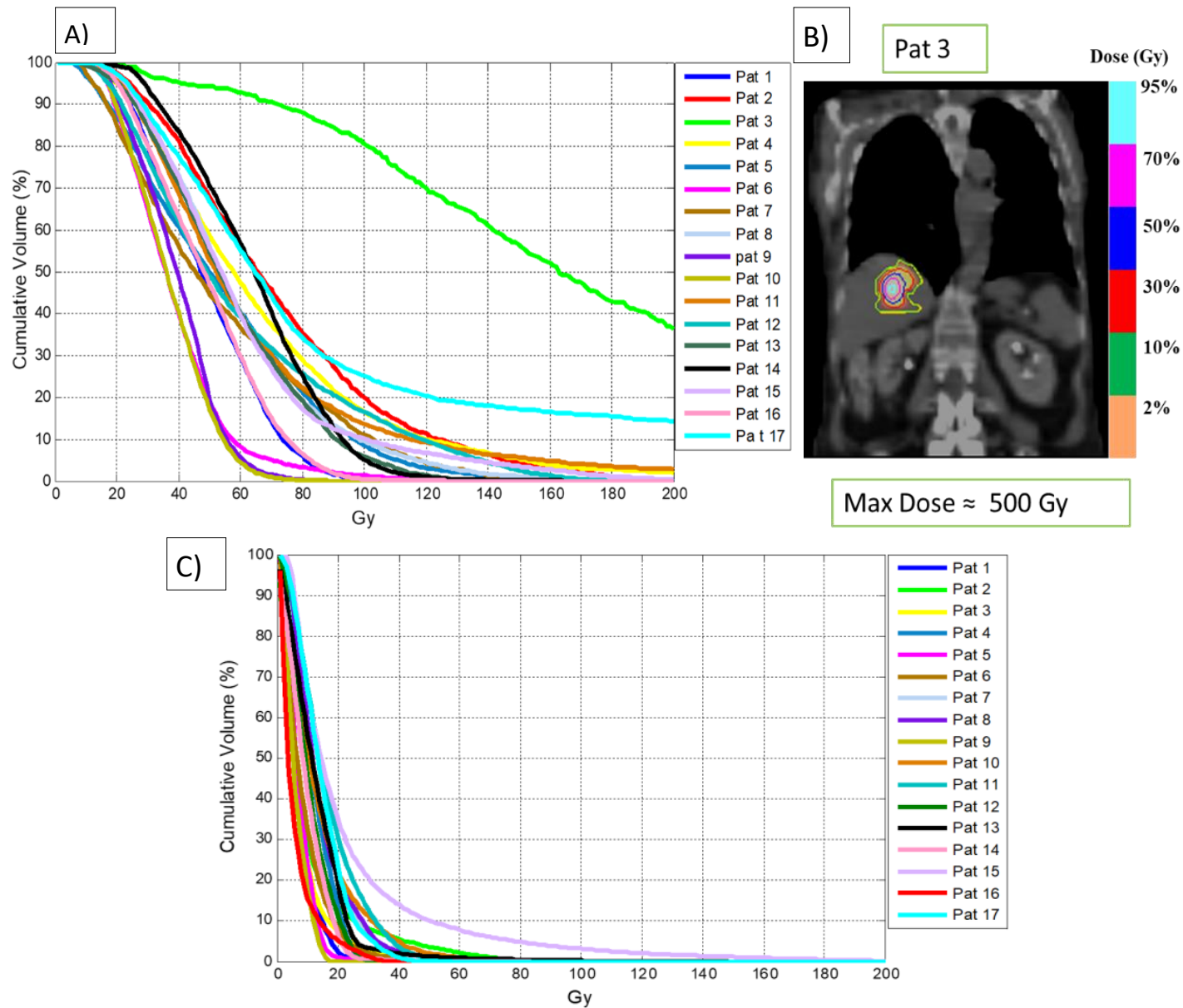

Figure 53: cDVH of tumor (A), fused isodose lines and CT scan for patient 3 (B) and cDVH of liver VOIs for all patients(C).

Dose distribution for the pre and post-treatment SPECT/CT images for patients who showed equal, two and three times of TRLs in their ${ }^{99 \mathrm{~m}} \mathrm{Tc}-\mathrm{MAA}$ scan than the ${ }^{90} \mathrm{Y}$ microsphere uptake (Table 13) is shown in Fig. 54. The figure shows the percent distributions in accordance to the maximum absorbed dose. The maximum ${ }^{90} \mathrm{Y}$ microsphere uptake were 88,142 and $1161 \mathrm{~Gy}$ for patient 9, 13 and 17 respectively. 
Table 15: Tumor volumes and TLRs for pre and post-treatment SPECT/CT images.

\begin{tabular}{|l|c|c|c|c|}
\hline \multirow{2}{*}{ Pat } & \multicolumn{2}{|c|}{ Tumor volume (ml) } & \multicolumn{2}{c|}{ TLR } \\
\cline { 2 - 5 } & ${ }^{99 \mathrm{~m}}$ Tc-MAA & ${ }^{90}$ Y microsphere & ${ }^{99 \mathrm{~m}}$ Tc-MAA & ${ }^{90}$ Y microsphere \\
\hline Pat 9 & 757 & 865 & 2.7 & 2.6 \\
\hline Pat 13 & 725 & 1008 & 10.2 & 5.3 \\
\hline Pat 17 & 271 & 403 & 14.7 & 5.0 \\
\hline
\end{tabular}
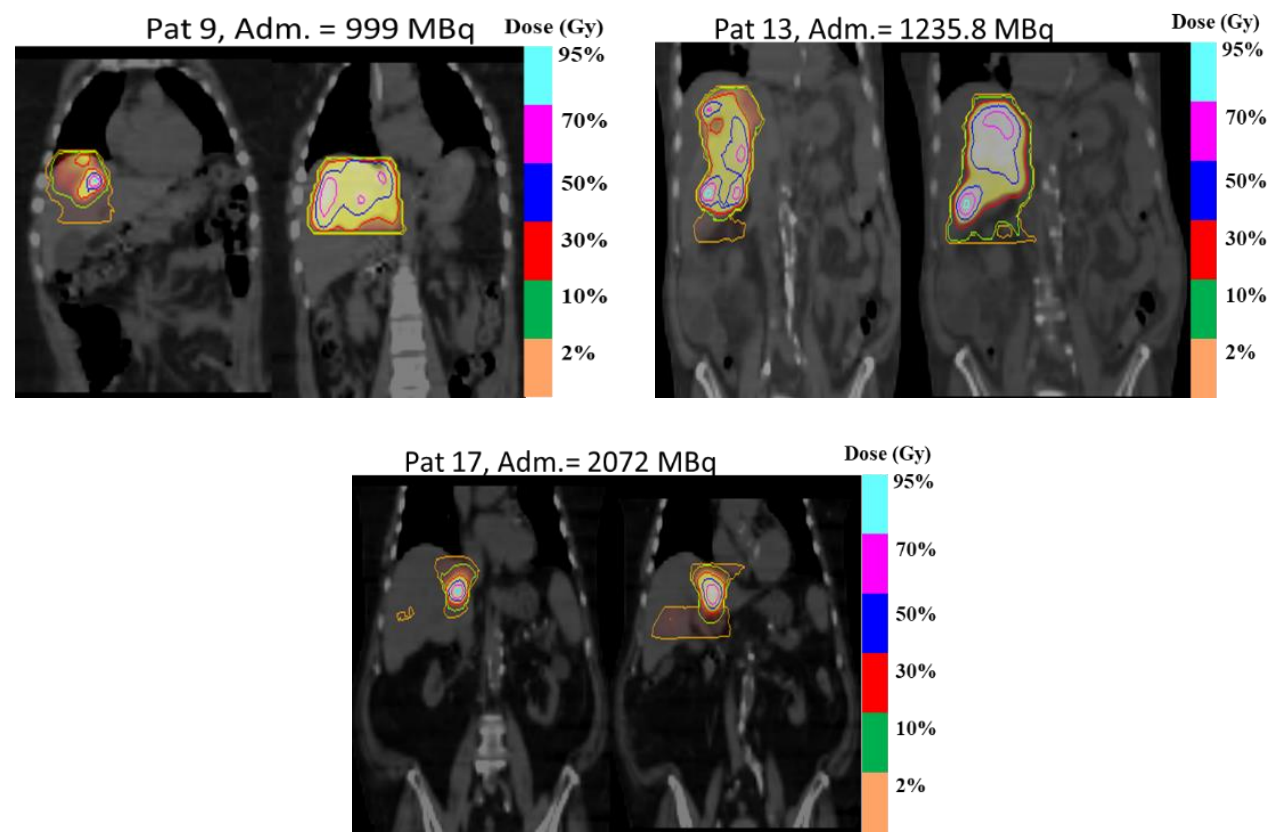

Figure 54: Fused isodose curves with the SPECT/CT of ${ }^{99 \mathrm{~m} T c-M A A}$ (left) and ${ }^{90} \mathrm{Y}$ microsphere (right).

Comparison of the dose distribution between images corrected for collimator blur using the developed method and those uncorrected showed a significant difference in the isodose curves whilst the overall difference in the cDVH per tissue volume is small, example shown in Fig. 55. Figure 56 shows a similar comparison for the same patient where the $S$ values for $4.8 \mathrm{~mm}$ were used for the method referred as the MC method. The dose distributions from both methods are similar but the major difference in the cDVH illustrated the actual absorbed doses per tissue volume are significantly different. 

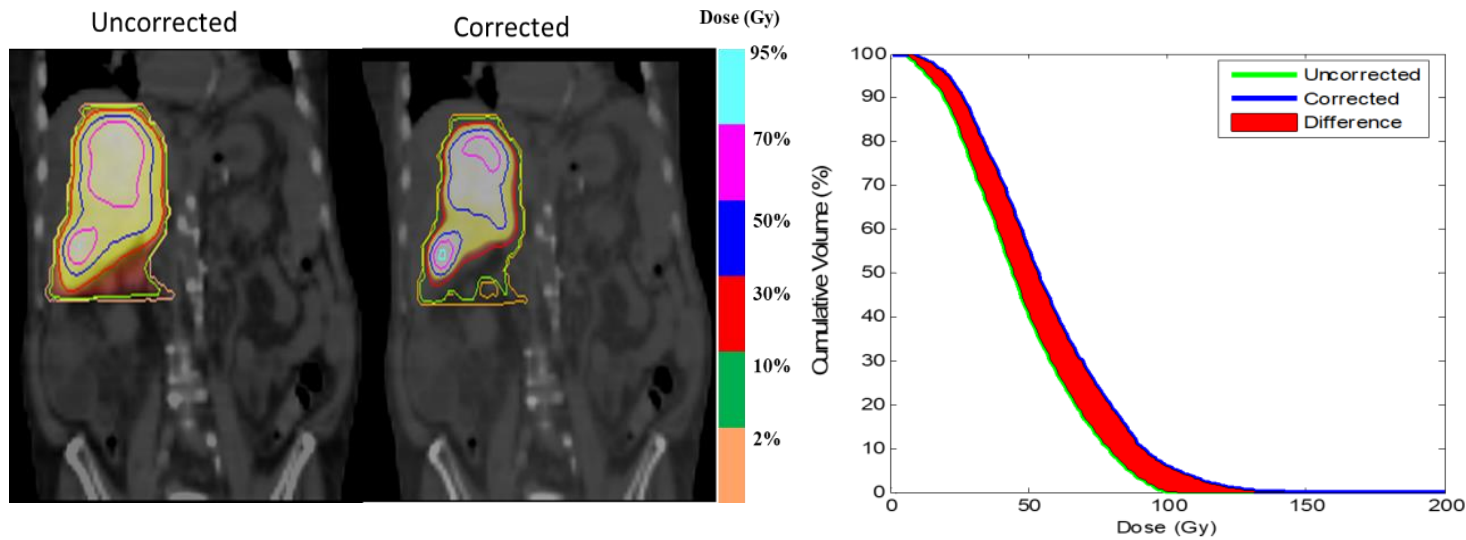

Figure 55: Dose distribution between ${ }^{90} \mathrm{Y}$ microsphere images before and after correction for collimator detector effect (left) and difference in cDVH (right).
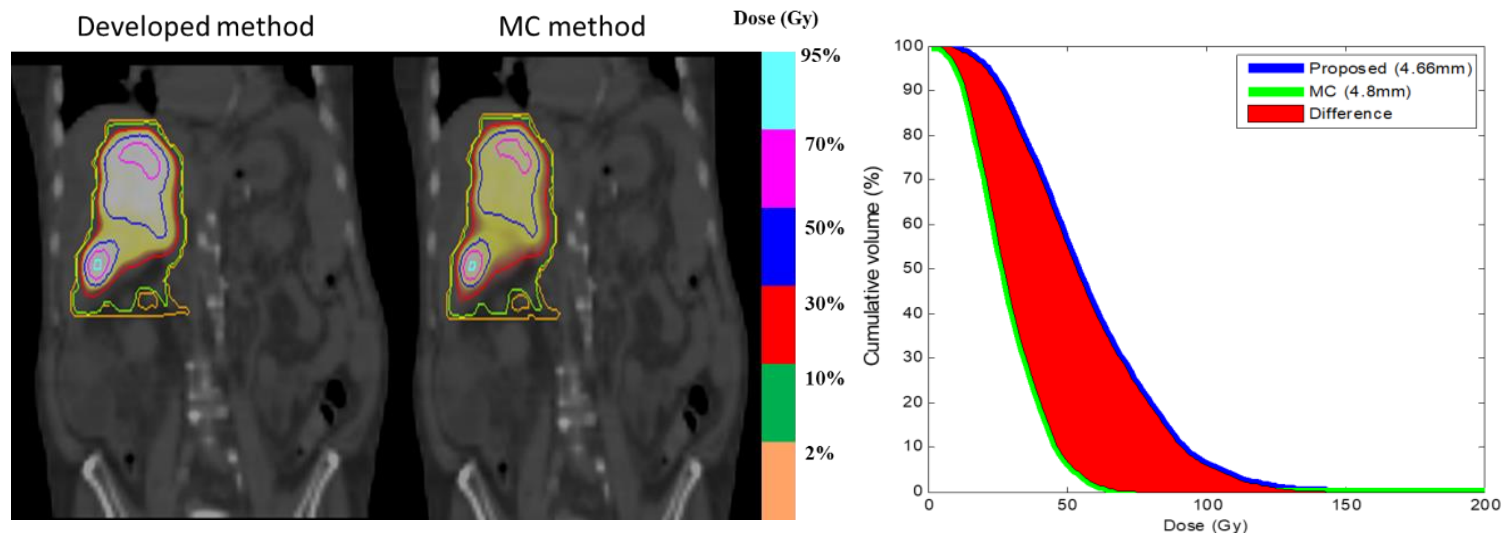

Figure 56: Comparison of dose distribution calculated using developed and the MC method on ${ }^{90} \mathrm{Y}$ microsphere SPECT/CT images (left) and the resulting difference in $\mathrm{cDVH}$ (right).

Figure 57 demonstrates the relationship between the administered activities and absorbed doses, there is a weak correlation with tumor absorbed dose $(\mathrm{r}=0.5, \mathrm{p}>0.05)$ but a strong correlation with healthy liver absorbed dose $(r=0.8, p<0.05)$. From the figure, it is observed that one patient appears as an outlier. Although the patient shouldn't be considered an outlier, since the administered activity (x-axis) is substantially higher than the rest of the patients, i.e. the higher absorbed dose in the liver and the tumor is valid considering the amount of administered activity. For the sake of completeness, we excluded this patient and analyzed the relationship between the administered activity and the absorbed doses in tumor and liver volumes. The result of this analysis 
gave a linear relationship between administered activity and liver dose $(r=0.52, p=0.1)$ but the higher $\mathrm{p}$ value suggests that the correlation is not dependable as the sample size is small. And no correlation between the administered activity and tumor absorbed dose was found $(\mathrm{r}=0.21, \mathrm{p}=$ $0.54)$.

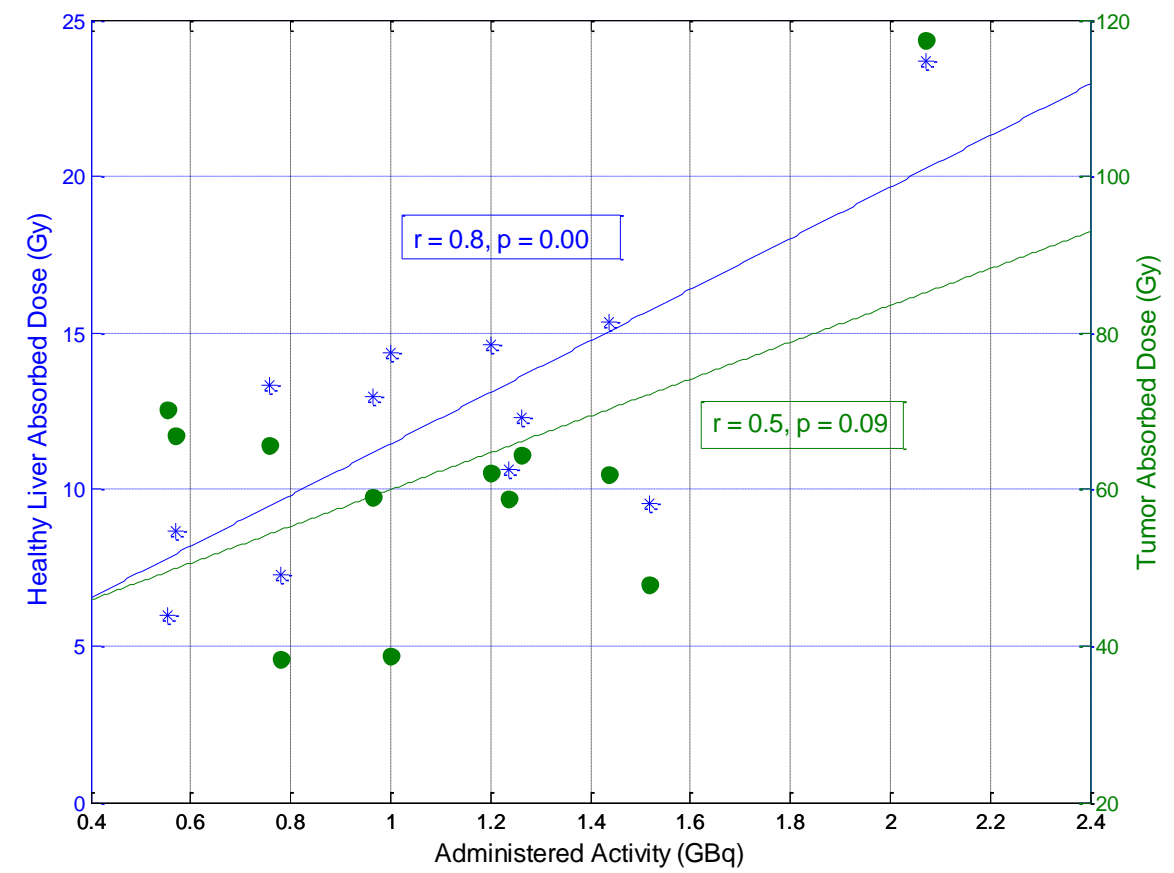

Figure 57: Correlation of administered activity with healthy liver and tumor absorbed doses.

Accurate dosimetry requires accurate voxel $\mathrm{S}$ values for the image voxel size. For our work, we used linear interpolation to estimate the voxel S values appropriate for the SPECT images. The most accurate method of estimating a voxel $\mathrm{S}$ value is using a direct Monte Carlo method for the exact voxel sizes of images at hand. Although using Monte Carlo machine isn't part of this dissertation, we compared dosimetry results between our developed method and $\mathrm{S}$ values generated using the MC method for a voxel size of $4.8 \mathrm{~mm}$, closer to our voxel size $(4.66 \mathrm{~mm})$. For as low as less than $0.2 \mathrm{~mm}$ difference in voxel edge sizes between the two methods, the reported mean dose values in the VOIs was substantial with the MC method giving smaller values. For ${ }^{90} \mathrm{Y}$, with mean beta particles range of $2.5 \mathrm{~mm}$, almost half the distance between the centroid of two voxels 
$(4.66 \mathrm{~mm})$, taking a large voxel edge size $(4.8 \mathrm{~mm})$ undermines the effect of the dose from beta particles that traveled shorter distances, giving an overall smaller dose estimate at the target voxels, i.e. undermining the effect of the immediate neighborhood voxel. Although there is no gold standard for validating dosimetry estimates, the MIRD method is considered the standard method of clinical dosimetry (88). Results of mean dosimetry from our method and the MIRD partition model based estimate is comparable for most of the patients. It should be emphasized here that the parameters used, such as TRL, tumor and liver volumes were similar in both methods in addition to the fact that there is only one tumor volume per patient. But the result might be different if the parameters were estimated for individual tumors as the TLR is dependent on overall counts/activity within a volume.

For the images uncorrected and corrected for spatial resolution recovery, the mean dose estimate within target VOIs is higher for the latter one as the spatial resolution recovery is based on deconvolution giving a more heterogeneous isodose distribution.

Comparing the pre and post-treatment dosimetry, for the small cohort patient data we studied we found no associations between TLRs and dose distributions. The expectation of equal TLR predicted from the ${ }^{99 \mathrm{~m}} \mathrm{Tc}-\mathrm{MAA}$ study and that calculated from $90 \mathrm{Y}$ microsphere uptake is taken as a guarantee of treatment success (10). But this expectation doesn't put into consideration the actual dose distribution between the two images. A study by Wondergem et al. (72) stated that ${ }^{99 \mathrm{~m}} \mathrm{Tc}-\mathrm{MAA}$ poorly predicts the distribution of ${ }^{90} \mathrm{Y}$ resin microspheres. The authors used the BlanAltman analysis to compare the distribution of the two activities. The study included patients with catheter positions similar in both planning and treatment procedure as well as those where the positions of the catheter was different in the two sessions. The authors found that the positions of the catheter tip during administrations significantly influences the disagreements and concluded that ${ }^{99 m}$ Tc-MAA doesn't accurately predict final ${ }^{90} \mathrm{Y}$ microsphere distribution. In our study, we 
compared the two procedures in terms of TRL as well as activity distribution. Our conclusion is that TLR shouldn't necessarily be the one parameter to evaluate the predictive accuracy of ${ }^{99 \mathrm{~m}} \mathrm{Tc}-$ MAA on the actual ${ }^{90} \mathrm{Y}$ microsphere treatment outcome.

We found no correlation between tumor dosimetry and administered activity while the liver dosimetry increased with an increase in the administered dose. This finding is justifiable as the administered activity for ${ }^{90} \mathrm{Y}$ resin (SIR-Spheres) microspheres treatment is based on the body surface area (BSA) method where the prescribed activity is adjusted based on the extent of tumor involvement in the liver and size of the patient (Eqn. 11). Thus, an increase in the administered activity may not result in an increased tumor uptake but a higher activity in the overall liver region.

\subsection{Conclusion}

A dosimetry algorithm that considers the non-uniform ${ }^{90} \mathrm{Y}$ microsphere distribution has been developed. The algorithm provides a 3D estimation of full radiation dose distribution within liver and the surrounding organs. The algorithm improves reproducibility of radiation dose calculation and solely depends on the specific patient image as opposed to relying on a standard human organ size which doesn't reflect individual patient biokinetic and response.

\section{Summary, Limitations and Future work}

${ }^{90} \mathrm{Y}$ is pure beta emitter which makes it an effective radionuclide for radioimmunotherapy of cancer increasing the radiation dose to tumor from the high energy beta particles. However, due to their short range, these particles can't be directly detected outside of the patient body for quantitative imaging. Instead secondary photons emitted as the result of the interaction between the beta particles and tissue are detected. SPECT/CT imaging utilizes bremsstrahlung photons emitted as a result of this interaction while PET/CT uses the small portion of annihilation events for assessing radionuclide distribution. ${ }^{90} \mathrm{Y}$ bremsstrahlung has a continuous energy spectrum resulting in scattered and septal penetration of photons during detection causing very poor image quality. 
On the other hand, the very small annihilation events for ${ }^{90} \mathrm{Y}$ PET/CT imaging has strong presence of random and scattered coincidences from the associated bremsstrahlung photons.

The first goal of this dissertation has been to employ a more realistic technique to improve the quantitative quality of ${ }^{90} \mathrm{Y}$ bremsstrahlung SPECT/CT and PET/CT images for dosimetry purposes. The post-reconstruction quantitative image improvement technique corrects image degradation due to collimator-detector response. The method considers the types of noises present in nuclear medicine imaging making it suitable for both SPECT and PET modalities. The only factor that interchanges between the modalities is the FWHM. Attenuation and scatter correction were performed using the manufacturers' software provided for each modality. The challenges for our method were image degradation due to object scatter, septal penetration and backscatter. These shortcomings limit the improvement in ${ }^{90} \mathrm{Y}$ bremsstrahlung SPECT/CT imaging. However, these effects are not a major concern in PET/CT imaging due to the coincidence detection mechanism and the algorithm employed for attenuation and scatter correction. The challenge with PET/CT imaging is the actual representation of patient administered dose due to the reliance of the image accuracy on the activity concentration under study. Despite these challenges, validation of the developed algorithm using phantom studies and clinical images has shown meaningful quantitative improvement which allows accurate quantification of total administered dose to a patient for treatment outcome prediction. Future quantitative improvement of ${ }^{90} \mathrm{Y}$ bremsstrahlung with ease of clinical application that includes compensation for the scatter, septal penetration and backscatter will provide complete correction of the image degrading factors. A future ${ }^{90} \mathrm{Y}$ PECT/CT study with similar activity concentration as patients recruited for RMT should be performed to represent actual count statistics.

The second objective of the dissertation focused on comparing the uptake distribution between pre-treatment ${ }^{99 \mathrm{~m}} \mathrm{Tc}-\mathrm{MAA}$ and post-treatment ${ }^{90} \mathrm{Y}$ microsphere SPECT/CT images. 
Accurate pre-treatment estimation of microsphere distribution within the tumor and healthy liver is critical for the success of RMT. Very small and diffused tumors that cannot be delineated on CT images can be identified using the functional SPECT images. Due to this benefit, our comparison of uptake between the two images was based on tumors delineated using the SPECT images. However, a tumor delineation technique that considers the non-uniform microspheres distribution within a tumor was a challenge. In addition, proper matching of the two pre and post-treatment SPECT/CT images in three dimensions was also investigated. Correlation analysis of the uptake distribution between the two images was based on cumulative tumor volumes. This comparison gives the entire activity distribution within the liver. Individual tumor comparison of pre and posttreatment uptake would provide a more detailed analysis of the study which would require a tumor segmentation on the CT scan by a radiologist.

In the final part of this dissertation, we developed a 3D algorithm for estimating radiation absorbed dose in tumor and liver VOIs. The method employs the voxel S-value method to calculate cumulated dose in each voxel from surrounding voxels giving absorbed dose per tissue volume. A future study could determine the correlation between tumor and liver dose estimates obtained from 90Y microspheres bremsstrahlung SPECT/CT imaging with tumor response and liver toxicity. This correlation might help in future treatment planning by setting a linear relationship between administered dose and tumor response. However, tumor response depends on many factors, such as tumor size, vascularity (blood supply), and presence of necrotic core which are different from patient to patient. Thus, future research should also consider the patient specific factors for treatment planning. Increasing patient population by incorporating those with higher LSF in the future study will enhance the dosimetry result by giving a complete perspective of treatment response to absorbed dose. This would require collecting extra patient data such as follow up studies. For patients who undergo multiple RMT, analysis of the change in tumor vasculature 
should also be part of the study as the change might contribute to the overall activity distribution within the liver.

\section{Overall Conclusion}

Nuclear medicine quantitative methods in ${ }^{90} \mathrm{Y}$ bremsstrahlung SPECT/CT and PET/CT imaging were investigated and evaluated for improved quantitation and dosimetry in RMT. The proposed maximum likelihood iterative spatial resolution algorithm corrects for detector response and Poisson statistical error using the Bayes' theorem. Optimal iteration number (6 iteration) of the algorithm was found from phantom studies and the algorithm was applied on patient ${ }^{90} \mathrm{Y}$ microsphere SPECT/CT images. Improvement in contrast recovery coefficients was achieved both in phantom and patient images. Methods for estimation of calibration factor were designed and implemented for activity estimation from patient and phantom images. We found that the calibration factor estimated using counts within liver VOIs gave the best results. Appropriate image co-registration methods for proper alignment of ${ }^{99 \mathrm{~m} T c-M A A}$ and ${ }^{90} \mathrm{Y}$ microsphere SPECT/CT images were investigated and determined. Correlation of uptake distribution between pre and posttreatment RMT were studied and correlation was found between the ${ }^{99 \mathrm{~m}} \mathrm{Tc}-\mathrm{MAA}$ and ${ }^{90} \mathrm{Y}$ microsphere SPECT/CT images. An algorithm for radiation absorbed dose estimation was developed. The algorithm considers the non-uniform ${ }^{90} \mathrm{Y}$ microsphere distribution in RMT and estimates dose delivered in tissue per volume at a voxel level. The method was implemented in patient ${ }^{90} \mathrm{Y}$ microsphere SPECT/CT images and isodose distributions were identified and aligned with the anatomical locations. In addition, dose volume histogram was generated for liver and tumor VOIs. For the moderate sample size (12 patients) studied, we found no significant similarity between the results of TRL and dose distribution on ${ }^{99 \mathrm{~m}} \mathrm{Tc}-\mathrm{MAA}$ and ${ }^{90} \mathrm{Y}$ microsphere SPECT/CT images. This result highlights the need for more than one parameter to justify the predictive accuracy of ${ }^{99 \mathrm{~m}} \mathrm{Tc}-\mathrm{MAA}$ for subsequent treatment outcome of ${ }^{90} \mathrm{Y}$ microspheres. Correlation 
between administered activity and absorbed doses in tumor and healthy liver was studied. The absorbed dose in tumors didn't show a linear relationship with the administered activity, while healthy liver absorbed dose increased with administered activity. 


\section{References}

1. Hamami ME, Poeppel TD, Müller S, et al. SPECT/CT with 99mTc-MAA in radioembolization with $90 \mathrm{Y}$ microspheres in patients with hepatocellular cancer. Journal of Nuclear Medicine. 2009;50:688-692.

2. Stubbs R, Wickremesekera S. Selective internal radiation therapy (SIRT): a new modality for treating patients with colorectal liver metastases. Hpb. 2004;6:133-139.

3. Fong AJ, Kemeny N, Brown K. Regional Transvascular Therapies for Liver Cancer: Theory, History, Evolution, and Current Options. Journal of Interventional Oncology. 2009;2.

4. Westcott MA, Coldwell DM, Liu DM, Zikria JF. The development, commercialization, and clinical context of yttrium-90 radiolabeled resin and glass microspheres. Advances in Radiation Oncology. 2016;1:351-364.

5. Kennedy A, Nag S, Salem R, et al. Recommendations for radioembolization of hepatic malignancies using yttrium-90 microsphere brachytherapy: a consensus panel report from the radioembolization brachytherapy oncology consortium. International Journal of Radiation Oncology* Biology* Physics. 2007;68:13-23.

6. Gulec S, Selwyn R, Weiner R. Nuclear medicine guidelines for radiomicrosphere therapy using Y-90 microspheres in patients with primary and metastatic liver cancer. $J$ Interventional Oncology. 2009;2:26-39.

7. Walrand S, Flux GD, Konijnenberg MW, et al. Dosimetry of yttrium-labelled radiopharmaceuticals for internal therapy: $86 \mathrm{Y}$ or $90 \mathrm{Y}$ imaging? European journal of nuclear medicine and molecular imaging. 2011;38:57-68.

\section{SIRTEX. www.sirtex.com.}

9. Garin E, Lenoir L, Rolland Y, et al. Dosimetry based on $99 \mathrm{mTc}$-macroaggregated albumin SPECT/CT accurately predicts tumor response and survival in hepatocellular carcinoma patients treated with 90Y-loaded glass microspheres: preliminary results. J Nucl Med. 2012;53:255-263.

10. Ilhan H, Goritschan A, Paprottka P, et al. Predictive Value of 99mTc-MAA SPECT for 90Y-Labeled Resin Microsphere Distribution in Radioembolization of Primary and Secondary Hepatic Tumors. J Nucl Med. 2015;56:1654-1660.

11. Riaz A, Awais R, Salem R. Side effects of yttrium-90 radioembolization. Frontiers in oncology. 2014;4. 
12. Shen S, DeNardo GL, Yuan A, DeNardo DA, DeNardo SJ. Planar gamma camera imaging and quantitation of yttrium-90 bremsstrahlung. energy. 1994;395:0-42.

13. Ito $\mathrm{S}$, Kurosawa $\mathrm{H}$, Kasahara $\mathrm{H}$, et al. $90 \mathrm{Y}$ bremsstrahlung emission computed tomography using gamma cameras. Annals of nuclear medicine. 2009;23:257-267.

14. Minarik D, Sjogreen Gleisner K, Ljungberg M. Evaluation of quantitative (90)Y SPECT based on experimental phantom studies. Phys Med Biol. 2008;53:5689-5703.

15. Rong X, Du Y, Ljungberg M, Rault E, Vandenberghe S, Frey EC. Development and evaluation of an improved quantitative $90 \mathrm{Y}$ bremsstrahlung SPECT method. Medical physics. 2012;39:2346-2358.

16. Rault E, Staelens S, Van Holen R, De Beenhouwer J, Vandenberghe S. Fast simulation of yttrium-90 bremsstrahlung photons with GATE. Medical physics. 2010;37:2943-2950.

17. Elschot M, Vermolen BJ, Lam MG, de Keizer B, van den Bosch MA, de Jong HW. Quantitative comparison of PET and Bremsstrahlung SPECT for imaging the in vivo yttrium-90 microsphere distribution after liver radioembolization. PLoS One. 2013;8:e55742.

18. Yue J, Mauxion T, Reyes DK, et al. Comparison of quantitative Y-90 SPECT and nontime-of-flight PET imaging in post-therapy radioembolization of liver cancer. Med Phys. 2016;43:5779.

19. Siman W, Mikell JK, Kappadath SC. Practical reconstruction protocol for quantitative (90)Y bremsstrahlung SPECT/CT. Med Phys. 2016;43:5093.

20. Pacilio M, Ferrari M, Chiesa C, et al. Impact of SPECT corrections on 3D-dosimetry for liver transarterial radioembolization using the patient relative calibration methodology. Med Phys. 2016;43:4053.

21. Rollo FD. Nuclear medicine physics, instrumentation, and agents. 1977.

22. Sharp PF, Gemmell HG, Murray AD. Practical nuclear medicine: Springer; 2005.

23. Cherry SR, Sorenson JA, Phelps ME. Physics in nuclear medicine: Elsevier Health Sciences; 2012.

24. Nuclear. http://nuclearmunkee.blogspot.com/2012/05/theraspheres.html 
25. Keidar Z, Israel O, Krausz Y. SPECT/CT in tumor imaging: technical aspects and clinical applications. Paper presented at: Seminars in nuclear medicine, 2003.

26. Zaidi H, Erwin WD. Quantitative analysis in nuclear medicine imaging. Soc Nuclear Med; 2007.

27. Attix FH. Introduction to radiological physics and radiation dosimetry: John Wiley \& Sons; 2008.

28. Frey E, Tsui B. Collimator-detector response compensation in SPECT. Quantitative analysis in nuclear medicine imaging: Springer; 2006:141-166.

29. Liang Z, Turkington T, Gilland D, Jaszczak R, Coleman R. Simultaneous compensation for attenuation, scatter and detector response for SPECT reconstruction in three dimensions. Physics in Medicine and Biology. 1992;37:587.

30. Flux G, Bardies M, Monsieurs M, Savolainen S, Strand S-E, Lassmann M. The impact of PET and SPECT on dosimetry for targeted radionuclide therapy. Zeitschrift für Medizinische Physik. 2006;16:47-59.

31. Gulec SA, Mesoloras G, Dezarn WA, McNeillie P, Kennedy AS. Safety and efficacy of Y-90 microsphere treatment in patients with primary and metastatic liver cancer: the tumor selectivity of the treatment as a function of tumor to liver flow ratio. J Transl Med. 2007;5:15.

32. Dewaraja YK, Frey EC, Sgouros G, et al. MIRD pamphlet no. 23: quantitative SPECT for patient-specific 3-dimensional dosimetry in internal radionuclide therapy. Journal of Nuclear Medicine. 2012;53:1310-1325.

33. Dezarn WA, Cessna JT, DeWerd LA, et al. Recommendations of the American Association of Physicists in Medicine on dosimetry, imaging, and quality assurance procedures for 90Y microsphere brachytherapy in the treatment of hepatic malignancies. Medical physics. 2011;38:4824-4845.

34. Ahmadzadehfar H, Duan H, Haug AR, Walrand S, Hoffmann M. The role of SPECT/CT in radioembolization of liver tumours. European journal of nuclear medicine and molecular imaging. 2014;41:115-124.

35. Lhommel R, van Elmbt L, Goffette P, et al. Feasibility of 90Y TOF PET-based dosimetry in liver metastasis therapy using SIR-Spheres. European journal of nuclear medicine and molecular imaging. 2010;37:1654-1662. 
36. Riaz A, Lewandowski RJ, Kulik LM, et al. Complications following radioembolization with yttrium-90 microspheres: a comprehensive literature review. Journal of Vascular and Interventional Radiology. 2009;20:1121-1130.

37. Walrand S. Bremsstrahlung SPECT/CT. Clinical Applications of SPECT-CT: Springer; 2014:271-280.

38. Heard S, Flux GD, Guy MJ, Ott RJ. Monte Carlo simulation of/sup 90/Y Bremsstrahlung imaging. Paper presented at: Nuclear Science Symposium Conference Record, 2004 IEEE, 2004.

39. Rong X, Ghaly M, Frey EC. Optimization of energy window for $90 \mathrm{Y}$ bremsstrahlung SPECT imaging for detection tasks using the ideal observer with model-mismatch. Medical physics. 2013;40.

40. Qian W, Clarke LP. A restoration algorithm for P-32 and Y-90 bremsstrahlung emission nuclear imaging: A wavelet-neural network approach. Medical physics. 1996;23:1309-1323.

41. Siegel JA. Quantitative bremsstrahlung SPECT imaging: attenuation-corrected activity determination. Journal of nuclear medicine: official publication, Society of Nuclear Medicine. 1994;35:1213-1216.

42. Ljungberg M, Frey E, Sjögreen K, Liu X, Dewaraja Y, Strand S-E. 3D absorbed dose calculations based on SPECT: evaluation for 111-In/90-Y therapy using Monte Carlo simulations. Cancer Biotherapy and Radiopharmaceuticals. 2003;18:99-107.

43. Center NND. http://www.nndc.bnl.gov/chart/reCenter.jsp?z=39\&n=51.

44. Beyer T, Townsend DW, Brun T, Kinahan PE. A combined PET/CT scanner for clinical oncology. The Journal of nuclear medicine. 2000;41:1369.

45. Khalil MM. Positron emission tomography (PET): Basic principles. Basic sciences of nuclear medicine: Springer; 2010:179-213.

46. imaging RfP. http://www.triumf.ca/radiochemistry-for-pet-imaging.

47. Agency IAE. http://www-pub.iaea.org/books/IAEABooks/10424/PET-CT-Atlas-onQuality-Control-and-Image-Artefacts.

48. Alessio AM, Kinahan PE, Cheng PM, Vesselle H, Karp JS. PET/CT scanner instrumentation, challenges, and solutions. Radiologic Clinics of North America. 2004;42:10171032 . 
49. Oliva MR, Saini S. Liver cancer imaging: role of CT, MRI, US and PET. Cancer imaging. 2004;4:S42-S46.

50. Niekel MC, Bipat S, Stoker J. Diagnostic Imaging of Colorectal Liver Metastases with CT, MR Imaging, FDG PET, and/or FDG PET/CT: A Meta-Analysis of Prospective Studies Including Patients Who Have Not Previously Undergone Treatment 1. Radiology. 2010;257:674-684.

51. Maliyekkel AT, Faulhaber PF. Liver PET/CT and Molecular Imaging. Abdominal Imaging. 2013:943-954.

52. Zealley IA, Skehan SJ, Rawlinson J, Coates G, Nahmias C, Somers S. Selection of Patients for Resection of Hepatic Metastases: Improved Detection of Extrahepatic Disease with FDG PET 1. Radiographics. 2001;21:S55-S69.

53. Teefey SA, Hildeboldt CC, Dehdashti F, et al. Detection of Primary Hepatic Malignancy in Liver Transplant Candidates: Prospective Comparison of CT, MR Imaging, US, and PET 1. Radiology. 2003;226:533-542.

54. Wolfort R, Papillion P, Turnage R, Lillien D, Ramaswamy M, Zibari G. Role of FDG-PET in the evaluation and staging of hepatocellular carcinoma with comparison of tumor size, AFP level, and histologic grade. International surgery. 2010;95:67.

55. Nickles RJ, Roberts AD, Nye JA, et al. Assaying and PET imaging of ytrrium-90: $1 / \mathrm{spl}$ Gt/34ppm> 0. Paper presented at: Nuclear Science Symposium Conference Record, 2004 IEEE, 2004.

56. Martí-Climent JM, Prieto E, Elosúa C, et al. PET optimization for improved assessment and accurate quantification of $90 \mathrm{Y}$-microsphere biodistribution after radioembolization. Medical physics. 2014;41.

57. Willowson K, Forwood N, Jakoby BW, Smith AM, Bailey DL. Quantitative 90Y image reconstruction in PET. Medical physics. 2012;39:7153-7159.

58. Attarwala AA, Molina-Duran F, Büsing K-A, et al. Quantitative and qualitative assessment of yttrium-90 PET/CT imaging. PLoS One. 2014;9:e110401.

59. Willowson KP, Tapner M, Bailey DL. A multicentre comparison of quantitative 90Y PET/CT for dosimetric purposes after radioembolization with resin microspheres. European journal of nuclear medicine and molecular imaging. 2015;42:1202-1222. 
60. Rowley LM, Bradley KM, Boardman P, Hallam A, McGowan DR. Optimization of Image Reconstruction for 90Y Selective Internal Radiotherapy on a Lutetium Yttrium Orthosilicate PET/CT System Using a Bayesian Penalized Likelihood Reconstruction Algorithm. Journal of Nuclear Medicine. 2017;58:658-664.

61. Beyer T, Freudenberg LS, Townsend DW, Czernin J. The future of hybrid imaging - part 1: hybrid imaging technologies and SPECT/CT. Insights into imaging. 2011;2:161-169.

62. Slomka PJ, Patton JA, Berman DS, Germano G. Advances in technical aspects of myocardial perfusion SPECT imaging. Journal of nuclear cardiology. 2009;16:255-276.

63. Tapp KN, Lea WB, Johnson MS, Tann M, Fletcher JW, Hutchins GD. The impact of image reconstruction bias on PET/CT 90Y dosimetry after radioembolization. Journal of Nuclear Medicine. 2014;55:1452-1458.

64. Richardson WH. Bayesian-based iterative method of image restoration. JOSA. 1972;62:5559.

65. Fish D, Walker J, Brinicombe A, Pike E. Blind deconvolution by means of the RichardsonLucy algorithm. JOSA A. 1995;12:58-65.

66. Elschot M, Lam MG, van den Bosch MA, Viergever MA, de Jong HW. Quantitative Monte Carlo-Based 90Y SPECT Reconstruction. Journal of Nuclear Medicine. 2013;54:1557-1563.

67. He B, Du Y, Segars WP, et al. Evaluation of quantitative imaging methods for organ activity and residence time estimation using a population of phantoms having realistic variations in anatomy and uptake. Medical physics. 2009;36:612-619.

68. Biodex. http://www.biodex.com/nuclear-medicine/products/phantoms.

69. Garin E, Rolland Y, Laffont S, Edeline J. Clinical impact of 99mTc-MAA SPECT/CTbased dosimetry in the radioembolization of liver malignancies with $90 \mathrm{Y}$-loaded microspheres. European journal of nuclear medicine and molecular imaging. 2016;43:559-575.

70. Gnesin S, Canetti L, Adib S, et al. Partition Model-Based 99mTc-MAA SPECT/CT Predictive Dosimetry Compared with 90Y TOF PET/CT Posttreatment Dosimetry in Radioembolization of Hepatocellular Carcinoma: A Quantitative Agreement Comparison. Journal of Nuclear Medicine. 2016;57:1672-1678.

71. Garin E, Rolland Y, Lenoir L, et al. Utility of quantitative 99mTc-MAA SPECT/CT for 90yttrium-labelled microsphere treatment planning: calculating vascularized hepatic volume and dosimetric approach. International journal of molecular imaging. 2011;2011. 
72. Wondergem M, Smits ML, Elschot M, et al. 99mTc-macroaggregated albumin poorly predicts the intrahepatic distribution of $90 \mathrm{Y}$ resin microspheres in hepatic radioembolization. Journal of Nuclear Medicine. 2013;54:1294-1301.

73. Penny WD, Friston KJ, Ashburner JT, Kiebel SJ, Nichols TE. Statistical parametric mapping: the analysis of functional brain images: Academic press; 2011.

74. Maes F, Vandermeulen D, Suetens P. Medical image registration using mutual information. Proceedings of the IEEE. 2003;91:1699-1722.

75. Boudraa A, Zaidi PDH. Image segmentation techniques in nuclear medicine imaging. Quantitative analysis in nuclear medicine imaging: Springer; 2006:308-357.

76. Chan TF, Vese LA. Active contours without edges. IEEE Transactions on image processing. 2001;10:266-277.

77. Chan TF, Vese LA. Active contour and segmentation models using geometric PDE's for medical imaging. Geometric methods in bio-medical image processing: Springer; 2002:63-75.

78. Bolch W, Bouchet L, Robertson J. The dosimetry of nonuniform activity distributions: radionuclide $\mathrm{S}$ values at the voxel level. Medical Internal Radiation Dose Committee. MIRD Pamphlet No. 17. J Nucl Med. 1999;40.

79. Gulec SA, Mesoloras G, Stabin M. Dosimetric techniques in 90Y-microsphere therapy of liver cancer: the MIRD equations for dose calculations. Journal of Nuclear Medicine. 2006;47:1209-1211.

80. Ho S, Lau W, Leung $\mathrm{T}$, et al. Partition model for estimating radiation doses from yttrium90 microspheres in treating hepatic tumours. European Journal of Nuclear Medicine and Molecular Imaging. 1996;23:947-952.

81. RADAR-Phantoms. http://www.doseinfo-radar.com/RADARphan.html.

82. Giap HB, Macey DJ, Bayouth JE, Boyer AL. Validation of a dose-point kernel convolution technique for internal dosimetry. Physics in medicine and biology. 1995;40:365.

83. Franquiz J, Chigurupati S, Kandagatla $\mathrm{K}$. Beta voxel $\mathrm{S}$ values for internal emitter dosimetry. Medical physics. 2003;30:1030-1032. 
84. Dieudonné A, Garin E, Laffont S, et al. Clinical feasibility of fast 3-dimensional dosimetry of the liver for treatment planning of hepatocellular carcinoma with 90Y-microspheres. Journal of Nuclear Medicine. 2011;52:1930-1937.

85. Medical-Imaging. http://www.medphys.it/.

86. 3DSLICER. https://www.slicer.org/.

87. Emami B. Tolerance of normal tissue to therapeutic radiation. Reports of radiotherapy and Oncology. 2013;1.

88. Gulec SA, Mesoloras G, Dezarn WA, McNeillie P, Kennedy AS. Safety and efficacy of Y-90 microsphere treatment in patients with primary and metastatic liver cancer: the tumor selectivity of the treatment as a function of tumor to liver flow ratio. Journal of translational medicine. 2007;5:15. 
VITA

SENAIT AKNAW DEBEBE

2008 Bachelors of Science in Electrical and Computer Engineering (summa cum laude)

Addis Ababa University Faculty of Technology

Addis Ababa, Ethiopia

2008-2009 Junior System Support Engineer

ZTE Corporation

Addis Ababa, Ethiopia

2011- Present Doctoral Candidate

Biomedical Engineering Department

Florida International University

Miami, FL

2011- Present Graduate Assistant: Teaching and Research Assistant

Biomedical Engineering Department

Florida International University

Miami, FL

\section{PUBLICATIONS AND PRESENTAIONS}

Debebe, S.A., Goryawala, M., Adjouadi, M., Mcgoron, A.J. and Gulec S.A, 2016. 18F-FLT Positron Emission Tomography/Computed Tomography Imaging in Pancreatic Cancer: Determination of Tumor Proliferative Activity and Comparison with Glycolytic Activity as Measured by 18F-FDG Positron Emission Tomography/Computed Tomography Imaging. Molecular Imaging and Radionuclide Therapy, 25(1), p.32.

Debebe, S.A., Adjouadi, M., Franquiz J., Gulec S.A. and Mcgoron, A.J. Yttrium-90 SPECT/CT Quantitative Study and Comparison of Uptake with Pretreatment 99mTc-MAA SPECT/CT in Radiomicrosphere Therapy, Under Review.

Debebe, S.A., Franquiz, J. and Mcgoron, A.J., 2015, March. 3D dosimetry estimation for selective internal radiation therapy (SIRT) using SPECT/CT images: a phantom study. In SPIE Medical Imaging (pp. 941234-941234). International Society for Optics and Photonics.

Debebe, S.A., Bhatt, R. and Mcgoron, A.J., 2013, May. Web based interactive medical imaging applications for teaching nuclear medicine. In Biomedical Engineering Conference (SBEC), 2013 29th Southern (pp. 133-134). IEEE. 
Debebe, S.A., Adjouadi, M., Franquiz J., Gulec S.A. and McGoron, A.J, Comparison of uptake distribution between Yttrium-90 SPECT/CT and pre-treatment Technetium-99m macroaggregated albumin (99mTc-MAA) SPECT/CT in Selective Internal Radiation Therapy, 59thAnnual AAPM Meeting, Denver, Colorado, July 2017.

Debebe, S.A., Goryawala, M., Adjouadi, M., Mcgoron, A.J. and Güleç, S.A. 18F-FLT Positron Emission Tomography/Computed Tomography Imaging in Pancreatic Cancer: Determination of Tumor Proliferative Activity and Comparison with Glycolytic Activity as Measured by 18F-FDG Positron Emission Tomography/Computed Tomography Imaging, Florida International University - Herbert Wertheim College of Medicine Second Student Research Symposium, Miami, Florida, April 2016.

Debebe, S.A., Franquiz, J. and McGoron, A.J., 3D dosimetry estimation for selective internal radiation therapy (SIRT) using SPECT/CT images: a phantom study, International Society for Optics and Photonics (SPIE) Medical Imaging, Orlando, Florida, February 2015.

Debebe, S.A., Adjouadi, M., Franquiz J. and Mcgoron, A.J., .Technetium-99m Compton window imaging; a trial for images generated from scattered photons. Graduate Research Day,

Department of Biomedical Engineering, Florida International University, Miami, Florida, November 2014.

Debebe, S.A., Bhatt, R. and McGoron, A.J., Web based interactive medical imaging applications for teaching nuclear medicine. 29th Southern Biomedical Engineering Conference, Miami, Florida, May 2013. 